SFB

Quantile cross-spectral measures of dependence

823 between economic variables

Jozef Baruník, Tobias Kley

$$
\text { Nr. 43/2015 }
$$

$\omega$

$\infty$

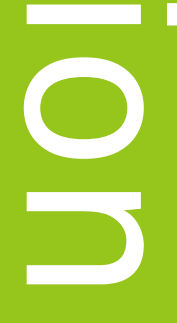

0

(1)

(D)

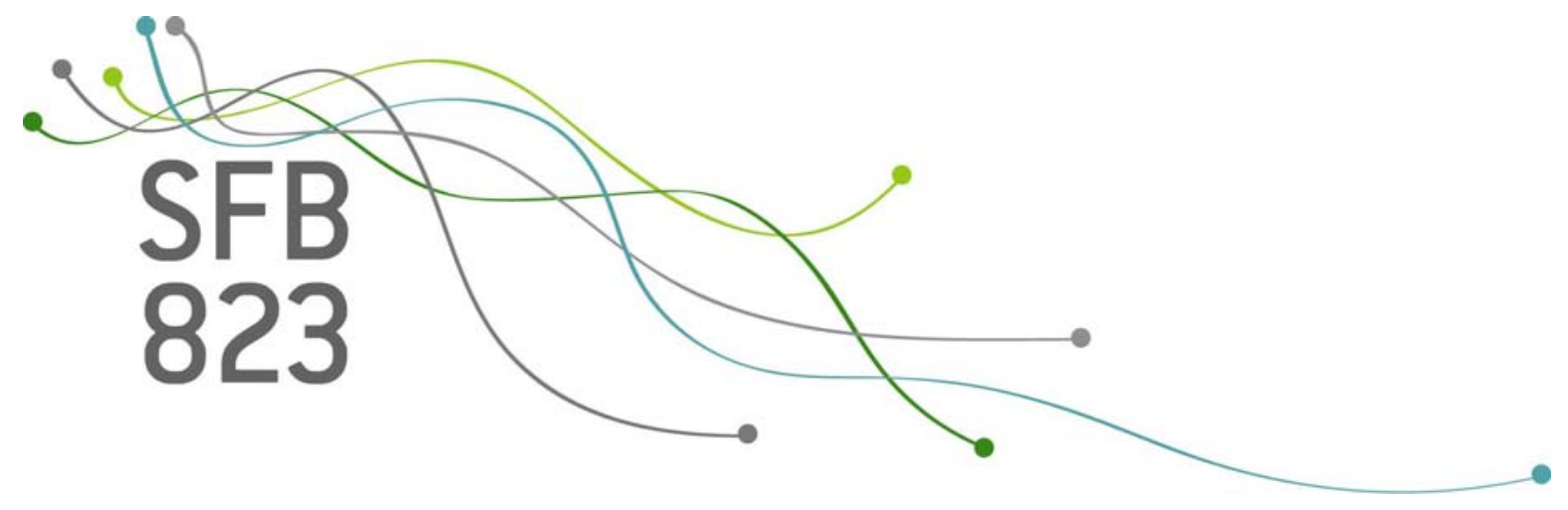





\title{
Quantile Cross-Spectral Measures of Dependence between Economic Variables*
}

\author{
Jozef BARUník ${ }^{\dagger}$ and Tobias KLEY
}

October 23, 2015

\begin{abstract}
In this paper we introduce quantile cross-spectral analysis of multiple time series which is designed to detect general dependence structures emerging in quantiles of the joint distribution in the frequency domain. We argue that this type of dependence is natural for economic time series but remains invisible when the traditional analysis is employed. To illustrate how such dependence structures can arise between variables in different parts of the joint distribution and across frequencies, we consider quantile vector autoregression processes. We define new estimators which capture the general dependence structure, provide a detailed analysis of their asymptotic properties and discuss how to conduct inference for a general class of possibly nonlinear processes. In an empirical illustration we examine one of the most prominent time series in economics and shed new light on the dependence of bivariate stock market returns.
\end{abstract}

Keywords: cross-spectral density, quantiles, dependence, time series, ranks, copula

*Authors are listed in alphabetical order, as they have equally contributed to the project. For estimation and inference of the quantile cross-spectral measures introduced in this paper, the $\mathrm{R}$ package quantspec is provided (Kley, 2014, 2015). The R package is available on https://cran.r-project. org/web/packages/quantspec/index.html

†The paper has been written during Jozef Baruník's postdoctoral stay with the Econometric Department, IITA, The Czech Academy of Sciences. Jozef Baruník is also Assistant Professor at the Institute of Economic Studies, Charles University in Prague, and gratefully acknowledges partial support from the European Union's Seventh Framework Programme (FP7/2007-2013) under grant agreement No. FP7-SSH- 612955 (FinMaP).

¥Tobias Kley is Postdoctoral Research Officer, Department of Statistics, London School of Economics and Political Science, London WC2A 2AE, UK. In May 2014 he obtained a PhD from the Fakultät für Mathematik, Ruhr-Universität Bochum, 44780 Bochum, Germany. He is grateful for being partially supported by the EPSRC fellowship "New challenges in time series analysis" (EP/L014246/1) and by the Collaborative Research Center "Statistical modeling of non-linear dynamic processes" (SFB 823, Teilprojekt C1) of the German Research Foundation (DFG). 


\section{Dependence structures in quantiles of the joint dis- tribution across frequencies}

One of the fundamental problems faced by a researcher in economics is how to quantify the dependence between economic variables. Although correlated variables are rather commonly observed phenomena in economics, it is often the case that strongly correlated variables under study are truly independent, and what we measure is mere spurious correlation (Granger and Newbold, 1974). Conversely, but equally deluding, uncorrelated variables may possess dependence in the different parts of the joint distribution, and/or at different frequencies. This dependence stays hidden when classical measures based on linear correlation and traditional cross-spectral analysis are used (Croux et al., 2001; Ning and Chollete, 2009; Fan and Patton, 2014). Hence, conventional models derived from averaged quantities as estimated by traditional measures may deliver rather misleading results.

In this paper, we introduce a new class of cross-spectral densities that characterize the dependence in quantiles of the joint distribution across frequencies. Subsequently, related quantities to which we will refer to as quantile coherency and quantile coherence are similarly defined and motivated as their traditional cross-spectral counterparts. Yet, instead of quantifying dependence by averaging with respect to the joint distribution, the new measures detect common behavior of variables in a specified part of their joint distribution. Hence, they are designed to detect any general type of dependence structure that may arise between variables under study.

Such complex dynamics may arise naturally in many (possibly multivariate) macroeconomic, or financial time series such as growth rates, inflation, housing markets, or stock market returns. In financial markets, extremely scarce and negative events in one asset can cause irrational outcomes and panics leading investors to ignore economic fundamentals and cause similarly extreme negative outcomes in other assets. In such situations, markets may be connected more strongly than in calm periods of small, or positive returns (Bae et al., 2003). Hence, the co-occurrences of large negative values may be more common across stock markets than co-occurrences of large positive values reflecting asymmetric behavior of economic agents. Moreover, long-term fluctuations in quantiles of the joint distribution may differ from the ones in the short-term due to differing risk perception of economic agents over distinct investment horizons. This behavior produces various degrees of persistence at different parts of the joint distribution, while on average the stock market returns remain impersistent. In univariate macroeconomic variables, researchers document asymmetric adjustment paths (Neftci, 1984; Enders and Granger, 1998) as firms are more liable to increase than to decrease in prices. Asymmetric business cycle dynamics at different quantiles can be caused by positive shocks to output being more persistent than negative shocks (Beaudry and Koop, 1993). While output fluctuations are known to be persistent, Beaudry and Koop (1993) document less persistence at longer horizons. Such asymmetric dependence at different horizons can be shared by multiple variables. Because classical, covariance-based approaches only take averaged information into account, these types of dependence fail to be identified by traditional means. Revealing such dependence structures, quantile cross-spectral analysis 
(a)

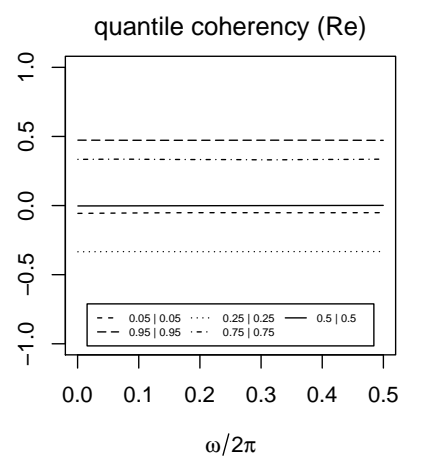

(b)

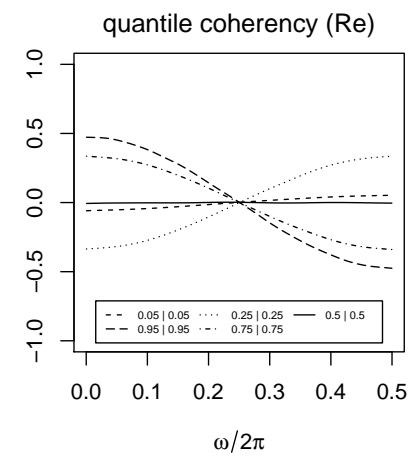

(c)

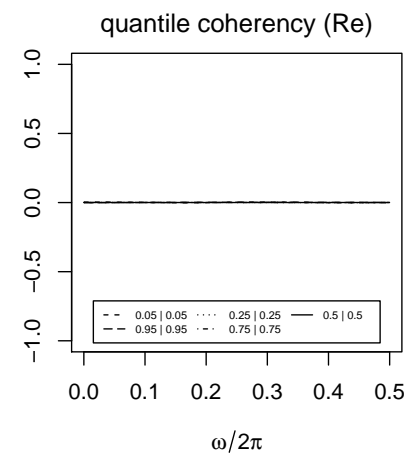

Figure 1: Illustration of dependence between processes $x_{t}$ and $y_{t}$ with $\operatorname{Cov}\left(x_{t}, y_{s}\right)=0$; with $\left(\epsilon_{t}\right)$ i.i.d. $\mathrm{N}(0,1)$ we have in (a) $x_{t}=\epsilon_{t}$ and $y_{t}=\epsilon_{t}^{2}$, in (b) $x_{t}=\epsilon_{t}$ and $y_{t}=\epsilon_{t-1}^{2}$, and in (c) $x_{t}, y_{t} \sim N(0,1)$ independent. All plots show real parts of the quantile coherency for $\tau_{1}=\tau_{2} \in\{0.05,0.25,0.5,0.75,0.95\}$ quantiles.

introduced in this paper can fundamentally change the way how we view the dependence between economic time series, and open new possibilities for the modeling of interactions between economic and financial variables.

Three toy examples illustrating the potential offered by quantile cross-spectral analysis are depicted in Figure 1. In each example one distinct type of dependence is considered: cross-sectional dependence in (a), serial dependence in (b), and independence in (c). We consider two processes that possess the desired dependence structure, but are indistinguishable in terms of traditional coherency. In the examples, $\left(\epsilon_{t}\right)$ is an independent sequence of standard normally distributed random variables. In column (a) of Figure 1 the dependence emerging between $\epsilon_{t}$ and $\epsilon_{t}^{2}$ is depicted. It is important to observe that $\epsilon_{t}$ and $\epsilon_{s}^{2}$ are uncorrelated ${ }^{1}$ Therefore, traditional coherency for $\left(\epsilon_{t}, \epsilon_{t}^{2}\right)$ would read zero across all frequencies, even though it is obvious that $\epsilon_{t}$ and $\epsilon_{t}^{2}$ are dependent. From the newly introduced quantile coherency, this dependence can easily be observed. More precisely, we can distinguish various degrees of dependence for each "part of the distribution". For example, there is no dependence in the center of the distribution (i. e., 0.5|0.5), but when one of the quantile levels is different from 0.5 the dependence is visible ${ }^{2}$ In this example the quantile coherency is constant across frequencies, which corresponds to the fact that there is no serial dependence. In column (b) of Figure 1 the process $\left(\epsilon_{t}, \epsilon_{t-1}^{2}\right)$ is studied, where we have introduced a time lag. Intuitively, the dependence in quantiles of this bivariate process will be the same as in example (a) in the long run, referring to frequencies close to zero. With increasing frequency, dependence will decline or incline gradually to values with opposite signs, as high frequency movements are in opposition due to the lag shift. This is clearly captured by quantile coherency, while the dependence structure would stay hidden away from traditional coherency, again, as it averages the dependence across quantiles. We can think about these

\footnotetext{
${ }^{1}$ This holds for $s=t$ due to the symmetry of the marginal distribution and for $s \neq t$ due to the independence of $\left(\epsilon_{t}\right)$

${ }^{2}$ Note that all plots show real parts of the complex-valued quantities for illustratory purposes.
} 
processes as being spuriously independent. To demonstrate the behavior of the quantile coherency when the processes under consideration are truly independent, we observe in column (c) of Figure 1 the quantities for independent bivariate Gaussian white noise, where quantile coherency displays zero dependence at all quantiles and frequencies, as expected. These illustrations strongly support our claim that there is need for more general measures that can provide a better understanding of the dependence between variables. These very simple, yet illuminating motivating examples focus on uncovering dependence in uncorrelated variables. Later in the text, we provide a data generating process based on quantile vector autoregression, which is able to generate even richer dependence structures (cf. Section 4).

Quantile cross-spectral analysis bridges two literatures focusing separately on the dependence between variables in quantiles, and across frequencies. It thus provides a general, unifying framework for estimating dependence between economic time series. As noted in the early work of Granger (1966), the spectral distribution of an economic variable has a typical shape which distinguishes long-term fluctuations from short-term ones. These fluctuations point to economic activity at different frequencies (after removal of trend in mean, as well as seasonal components). After Granger (1966) studied the behavior of single time series, important literature using cross-spectral analysis to identify the dependence between variables quickly emerged (from Granger (1969) to more recent Croux et al. (2001)). Instead of considering only cross-sectional correlations, researchers started to use coherency (frequency dependent correlation) to investigate short-run and long-run dynamic properties of multiple time series, and identify business cycle synchronization (Croux et al., 2001). In one of his very last papers, Granger (2010) hypothesized about possible cointegrating relationships in quantiles, leading to the first notion of general types of dependence that quantile cross-spectral analysis is addressing. The quantile cointegration developed by Xiao (2009) partially addresses the problem, but does not allow to fully explore the frequency dependent structure of correlations in different quantiles of the joint distribution.

Although a powerful tool, classical (cross-)spectral analysis becomes of limited use in many situations. Relying entirely on means and covariances, it is not robust to heavy tails, cannot accommodate infinite variances, and cannot account for conditional changes in skewness or kurtosis, or dependence in extremes - features often present in economic data. Several authors aimed at a robustification of the classical tools against outliers (see Kleiner et al. (1979) for an early contribution, or Chapter 8 of Maronna et al. (2006) for an overview; a weighted "self-normalized" periodogram was introduced by Klüppelberg and Mikosch (1994); parameter estimates in time series models, based on the traditional spectra, were obtained from a robustified periodogram by Hill and McCloskey (2014); Katkovnik (1998) introduced a periodogram based on robust loss functions). Recently, important contributions that aim for accounting of more general dynamics emerged in the literature.

Measures as, for example, distance correlation (Székely et al., 2007) and martingale difference correlation (Shao and Zhang, 2014) go beyond traditional correlation and instead can indicate whether random quantities are independent or martingale differences, respectively. For time series, in the time domain, Zhou (2012) introduced auto distance correlations that are zero if and only if the measured time series components are inde- 
pendent. Linton and Whang (2007), and Davis et al. (2009) introduced the (univariate) concepts of quantilograms and extremograms, respectively. More recently, quantile correlation (Schmitt et al., 2015), and quantile autocorrelation functions (Li et al., 2015) together with cross-quantilograms (Han et al., 2014) have been proposed as a fundamental tools for analyzing dependence in quantiles of the distribution.

In the frequency domain, Hong (1999) introduced a generalized spectral density. In the generalized spectral density covariances are replaced by quantities that are closely related to empirical characteristic functions. In Hong (2000) the Fourier transform of empirical copulas at different lags is considered for testing the hypothesis of pairwise independence. Recently, under the names of Laplace-, quantile and copula spectral density and spectral density kernels, various quantile-related spectral concepts have been proposed, for the frequency domain. The approaches by Hagemann (2013) and Li (2008, 2012) are designed to consider cyclical dependence in the distribution at user-specified quantiles. Mikosch and Zhao (2014, 2015) define and analyze a periodogram (and its integrated version) of extreme events. As noted by Hagemann (2013) other approaches aim at discovering "the presence of any type of dependence structure in time series data", referring to work of Dette et al. (2015) and Lee and Rao (2012). This comment also applies to Kley et al. (2015). In the present paper our aim is to extend the most general of these approaches to multivariate time series, so that it can be employed in the analysis of not only the serial dependence of one, but also for the joint analysis of multiple economic time series.

While our main motivation for the development of the quantile cross-spectral analysis was to provide a tool to measure general dependence structures between economic variables that previously remained hidden from researchers, our results also constitute an important step in robustifying the traditional cross-spectral analysis. Quantile-based spectral quantities are very attractive as they do not require the existence of any moments, which is in sharp contrast to the classical assumptions, where moments up to the order of the cumulants have to exist. The rank-based estimators proposed in Section 2.4 of this paper are robust to many common violations of traditional assumptions found in data, including outliers, heavy tails, and changes in higher moments of the distribution. As we motivated earlier, it is even possible to reveal the dependence in uncorrelated data. As an essential ingredient for a successful applications, we provide a rigorous analysis of asymptotic properties and show that for a very broad class of processes (including the classical linear ARMA time series models, but also important nonlinear models such e.g., $\mathrm{ARCH}$ and GARCH), properly centered and smoothed versions of the quantile-based estimators converge in distribution to centered Gaussian processes. Based on these results, generalizing univariate quantile spectral analysis of Kley et al. (2015), we construct asymptotic pointwise confidence bands for the proposed quantities.

In order to support our theoretical discussions empirically, we study the dependence in one of the most prominent time series in economics - stock market returns. Quantile cross-spectral analysis of bivariate stock market returns detects commonalities in quantiles of the joint distribution of stock market returns across frequencies. We document strong dependence of the bivariate returns series in periods of large negative returns, which varies over frequencies. Positive returns display less dependence over all frequencies. This result is not favorable for an investor relying on traditional pricing theories, 
as he/she may want exactly the opposite situation: choosing to invest to a stock with independent negative returns, but dependent positive returns. Our tool can reveal if such systematic risk exists in quantiles of the joint distribution in the long-, medium-, or short-run investment horizons.

\section{Quantile cross-spectral quantities}

\subsection{Quantile cross-spectral density kernels}

Throughout the paper $\left(\boldsymbol{X}_{t}\right)_{t \in \mathbb{Z}}$ denotes a $d$-variate, strictly stationary process, with components $X_{t, j}, j=1, \ldots, d$; i. e. $\boldsymbol{X}_{t}=\left(X_{t, 1}, \ldots, X_{t, d}\right)$. The marginal distribution function of $X_{t, j}$ will be denoted by $F_{j}$, and by $q_{j}(\tau):=F_{j}^{-1}(\tau):=\inf \left\{q \in \mathbb{R}: \tau \leq F_{j}(q)\right\}, \tau \in[0,1]$, we denote the corresponding quantile function. We use the convention $\inf \emptyset=+\infty$, such that, if $\tau=0$ or $\tau=1$, then $-\infty$ and $+\infty$ are possible values for $q_{j}(\tau)$, respectively. We will write $\bar{z}$ for the complex conjugate, $\Re z$ for the real part and $\Im z$ for the imaginary part of $z \in \mathbb{C}$, respectively. The transpose of a matrix $\boldsymbol{A}$ will be denoted by $\boldsymbol{A}^{\prime}$, the inverse of a regular matrix $\boldsymbol{B}$ will be denoted by $\boldsymbol{B}^{-1}$.

As a measure for the serial and cross-dependency structure of $\left(\boldsymbol{X}_{t}\right)_{t \in \mathbb{Z}}$, we define the matrix of quantile cross-covariance kernels, $\Gamma_{k}\left(\tau_{1}, \tau_{2}\right):=\left(\gamma_{k}^{j_{1}, j_{2}}\left(\tau_{1}, \tau_{2}\right)\right)_{j_{1}, j_{2}=1, \ldots, d}$, where

$$
\gamma_{k}^{j_{1}, j_{2}}\left(\tau_{1}, \tau_{2}\right):=\operatorname{Cov}\left(I\left\{X_{t+k, j_{1}} \leq q_{j_{1}}\left(\tau_{1}\right)\right\}, I\left\{X_{t, j_{2}} \leq q_{j_{2}}\left(\tau_{2}\right)\right\}\right),
$$

$j_{1}, j_{2} \in\{1, \ldots, d\}, k \in \mathbb{Z}, \tau_{1}, \tau_{2} \in[0,1]$, and $I\{A\}$ denotes the indicator function of the event $A$. This quantile-based quantities and the ones to be defined in the sequel are functions of the two variables $\tau_{1}$ and $\tau_{2}$. They are thus richer in information than the traditional counterparts. We have added the term kernel to the name for the quantities to stress this fact, but will frequently omit it in the rest of the paper, for the sake of brevity.

For continuous $F_{j_{1}}$ and $F_{j_{2}}$, these quantities coincide with the difference of the copula of $\left(X_{t+k, j_{1}}, X_{t, j_{2}}\right)$ and the independence copula. Thus, they provide important information about both the serial dependence (by letting $k$ vary) and the cross-section-dependence (by choosing $j_{1} \neq j_{2}$ ). In the frequency domain this yields (under appropriate mixing conditions) the matrix of quantile cross-spectral density kernels

$$
\mathfrak{f}\left(\omega ; \tau_{1}, \tau_{2}\right):=\left(\mathfrak{f}^{j_{1}, j_{2}}\left(\omega ; \tau_{1}, \tau_{2}\right)\right)_{j_{1}, j_{2}=1, \ldots, d}
$$

where

$$
\mathfrak{f}^{j_{1}, j_{2}}\left(\omega ; \tau_{1}, \tau_{2}\right):=(2 \pi)^{-1} \sum_{k=-\infty}^{\infty} \gamma_{k}^{j_{1}, j_{2}}\left(\tau_{1}, \tau_{2}\right) \mathrm{e}^{-\mathrm{i} k \omega},
$$

$j_{1}, j_{2} \in\{1, \ldots, d\}, \omega \in \mathbb{R}, \tau_{1}, \tau_{2} \in[0,1]$. Observe that $\mathfrak{f}$ takes values in $\mathbb{C}^{d \times d}$ (the set of all complex-valued $d \times d$ matrices). Further, note that, as a function of $\omega$, but for fixed $\tau_{1}, \tau_{2}$, it coincides with the traditional cross-spectral density of the bivariate, binary process

$$
\left(I\left\{X_{t, j_{1}} \leq q_{j_{1}}\left(\tau_{1}\right)\right\}, I\left\{X_{t, j_{2}} \leq q_{j_{2}}\left(\tau_{2}\right)\right\}\right)_{t \in \mathbb{Z}}
$$


The time series in (3) has the bivariate time series $\left(X_{t, j_{1}}, X_{t, j_{2}}\right)_{t \in \mathbb{Z}}$ as a "latent driver" and indicates whether the values of the components $j_{1}$ and $j_{2}$ are below the respective marginal distribution's $\tau_{1}$ and $\tau_{2}$ quantile. Note that $\left(X_{t, j_{1}}, X_{t, j_{2}}\right)_{t \in \mathbb{Z}}$ are the $j_{1}$ th and $j_{2}$ th component of the time series $\left(\boldsymbol{X}_{t}\right)_{t \in \mathbb{Z}}$ under consideration.

In the special case of a univariate time series, i.e. pick one of the $d$ components $\left(X_{t, j}\right)_{t \in \mathbb{Z}}$ of $\left(\boldsymbol{X}_{t}\right)_{t \in \mathbb{Z}}$, quantities as in $(2)$, with $j_{1}=j_{2}=: j$, were previously considered in different versions. An integrated version of the spectrum defined in (2) was considered by Hong (2000) in the context of testing for independence. Li (2008) considered the special case of $\tau_{1}=\tau_{2}=0.5$ and later the more general case where $\tau_{1}=\tau_{2} \in(0,1)(\mathrm{Li}$, 2012). Under the name of $\tau$-th quantile spectral densities Hagemann (2013) also studied the special case where $\tau_{1}=\tau_{2}$ is required. The general case of the spectral density kernel as defined in (2), still for $j_{1}=j_{2}$, but allowing $\left(\tau_{1}, \tau_{2}\right) \in[0,1]^{2}$, was discussed in Dette et al. (2015), where consistent estimation based on quantile regression in an harmonic linear model is proposed. Dette et al. (2015) refer to the univariate version as the copula spectral density kernel to distinguish it from a weighted version that they call the Laplace spectral density kernel. An estimator based on the Fourier transform of indicator functions based on the ranks was recently discussed in Kley et al. (2015). Note the substantial difference between the two cases $\tau_{1}=\tau_{2} \in[0,1]$ and $\left(\tau_{1}, \tau_{2}\right) \in[0,1]^{2}$. The first case corresponds to analyzing the two components of the binary, bivariate time series (3) separately, while in the second case the analysis is performed jointly.

In this paper, by allowing $j_{1} \neq j_{2}$ in (3), the quantile cross-spectral density kernel further generalizes the copula spectral density defined in Dette et al. (2015), allowing for a detailed analysis of joint dynamics in the multivariate process $\left(\boldsymbol{X}_{t}\right)_{t \in \mathbb{Z}}$. More precisely, note the relation

$$
\int_{-\pi}^{\pi} \mathfrak{f}^{j_{1}, j_{2}}\left(\omega ; \tau_{1}, \tau_{2}\right) \mathrm{e}^{\mathrm{i} k \omega} \mathrm{d} \omega+\tau_{1} \tau_{2}=\mathbb{P}\left(X_{t+k, j_{1}} \leq q_{j_{1}}\left(\tau_{1}\right), X_{t, j_{2}} \leq q_{j_{2}}\left(\tau_{2}\right)\right) .
$$

The quantity on the right hand side of (4), as a function of $\left(\tau_{1}, \tau_{2}\right)$, is the copula of the pair $\left(X_{t+k, j_{1}}, X_{t, j_{2}}\right)$. The equality (4) thus shows how any of the pair copulas can be derived from the quantile cross-spectral density kernel defined in (2). Thus, the quantile crossspectral density kernel provides a full description of all copulas of pairs in the process. Comparing these new quantities with their traditional counterparts, it can be observed that covariances and means are essentially replaced by copulas and quantiles. Similar to the regression setting, where this approach provides valuable extra information (Koenker, 2005), the quantile-based approach to spectral analysis supplements the traditional $L^{2}-$ spectral analysis.

\subsection{Quantile coherency and coherence}

In the situation described in this paper, there exists a right continuous orthogonal increment process $\left\{Z_{j}^{\tau}(\omega):-\pi \leq \omega \leq \pi\right\}$, for every $j \in\{1, \ldots, d\}$ and $\tau \in[0,1]$, such that the Cramér representation

$$
I\left\{X_{t, j} \leq q_{j}(\tau)\right\}=\int_{-\pi}^{\pi} \mathrm{e}^{\mathrm{i} t \omega} \mathrm{d} Z_{j}^{\tau}(\omega)
$$


holds [cf., e.g., Theorem 1.2.15 in Taniguchi and Kakizawa (2000)]. Note the fact that $\left(X_{t, j}\right)_{t \in \mathbb{Z}}$ is strictly stationary and therefore $\left(I\left\{X_{t, j} \leq q_{j}(\tau)\right\}\right)_{t \in \mathbb{Z}}$ is second-order stationary, as the boundedness of the indicator functions implies existence of their second moments.

The quantile cross-spectral density kernels are closely related to these orthogonal increment processes [cf. (Brillinger, 1975, p. 101) and (Brockwell and Davis, 1987, p. 436)]. More specifically the following relation holds:

$$
\mathfrak{f}^{j_{1}, j_{2}}\left(\omega ; \tau_{1}, \tau_{2}\right) \mathrm{d} \omega=\operatorname{Cov}\left(\mathrm{d} Z_{j_{1}}^{\tau_{1}}(\omega), \mathrm{d} Z_{j_{2}}^{\tau_{2}}(\omega)\right)
$$

which is short for

$$
\int_{\omega_{1}}^{\omega_{2}} \mathfrak{f}^{j_{1}, j_{2}}\left(\omega ; \tau_{1}, \tau_{2}\right) \mathrm{d} \omega=\operatorname{Cov}\left(Z_{j_{1}}^{\tau_{1}}\left(\omega_{2}\right)-Z_{j_{1}}^{\tau_{1}}\left(\omega_{1}\right), Z_{j_{2}}^{\tau_{2}}\left(\omega_{2}\right)-Z_{j_{2}}^{\tau_{2}}(\omega)\right), \quad-\pi \leq \omega_{1} \leq \omega_{2} \leq \pi .
$$

It is important to observe that $\mathfrak{f}^{j_{1}, j_{2}}\left(\omega ; \tau_{1}, \tau_{2}\right)$ is complex-valued. One way to represent $\mathfrak{f}^{j_{1}, j_{2}}\left(\omega ; \tau_{1}, \tau_{2}\right)$ is to decompose it into its real and imaginary part. The real part is known as the cospectrum (of the processes $\left(I\left\{X_{t, j_{1}} \leq q_{j_{1}}\left(\tau_{1}\right)\right\}\right)_{t \in \mathbb{Z}}$ and $\left(I\left\{X_{t, j_{2}} \leq q_{j_{2}}\left(\tau_{2}\right)\right\}\right)_{t \in \mathbb{Z}}$ ). The negative of the imaginary part is commonly referred to as the quadrature spectrum. We will refer to these quantities as the quantile cospectrum and quantile quadrature

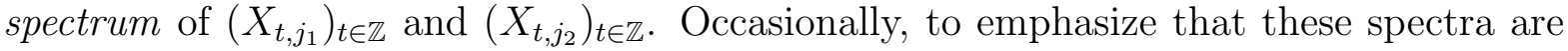
functions of $\left(\tau_{1}, \tau_{2}\right)$, we will refer to them as the quantile cospectrum kernel and quantile quadrature spectrum kernel, respectively. The quantile quadrature spectrum vanishes if $j_{1}=j_{2}$ and $\tau_{1}=\tau_{2}$. More generally, as described in Kley et al. (2015), for any fixed $j_{1}, j_{2}$, the quadrature spectrum will vanish, for all $\tau_{1}, \tau_{2}$, if and only if $\left(X_{t-k, j_{1}}, X_{t, j_{2}}\right)$ and $\left(X_{t+k, j_{1}}, X_{t, j_{2}}\right)$ possess the same copula, for all $k$.

An alternative way to look at $\mathfrak{f}^{j_{1}, j_{2}}\left(\omega ; \tau_{1}, \tau_{2}\right)$ is by representing it in polar coordinates. The radius $\left|\mathfrak{f}^{j_{1}, j_{2}}\left(\omega ; \tau_{1}, \tau_{2}\right)\right|$ is then referred to as the amplitude spectrum (of the two processes $\left(I\left\{X_{t, j_{1}} \leq q_{j_{1}}\left(\tau_{1}\right)\right\}\right)_{t \in \mathbb{Z}}$ and $\left.\left(I\left\{X_{t, j_{2}} \leq q_{j_{2}}\left(\tau_{2}\right)\right\}\right)_{t \in \mathbb{Z}}\right)$, while the angle $\arg \left(f^{j_{1}, j_{2}}\left(\omega ; \tau_{1}, \tau_{2}\right)\right)$ is the so called phase spectrum, respectively. We refer to these quantities as the quantile amplitude spectrum and the quantile phase spectrum of $\left(X_{t, j_{1}}\right)_{t \in \mathbb{Z}}$ and $\left(X_{t, j_{2}}\right)_{t \in \mathbb{Z}}$.

A closely related quantity that can be used as a measure for the dynamic dependence of the two processes $\left(X_{t, j_{1}}\right)_{t \in \mathbb{Z}}$ and $\left(X_{t, j_{2}}\right)_{t \in \mathbb{Z}}$ is the correlation between $\mathrm{d} Z_{j_{1}}^{\tau_{1}}(\omega)$ and $\mathrm{d} Z_{j_{2}}^{\tau_{2}}(\omega)$. We will call this quantity the quantile coherency kernel of $\left(X_{t, j_{1}}\right)_{t \in \mathbb{Z}}$ and $\left(X_{t, j_{2}}\right)_{t \in \mathbb{Z}}$ and denote it by

$$
\mathfrak{R}^{j_{1}, j_{2}}\left(\omega ; \tau_{1}, \tau_{2}\right):=\operatorname{Corr}\left(\mathrm{d} Z_{j_{1}}^{\tau_{1}}(\omega), \mathrm{d} Z_{j_{2}}^{\tau_{2}}(\omega)\right)=\frac{\mathfrak{f}^{j_{1}, j_{2}}\left(\omega ; \tau_{1}, \tau_{2}\right)}{\left(\mathfrak{f}^{j_{1}, j_{1}}\left(\omega ; \tau_{1}, \tau_{1}\right) \mathfrak{f}^{j_{2}, j_{2}}\left(\omega ; \tau_{2}, \tau_{2}\right)\right)^{1 / 2}},
$$

$\left(\tau_{1}, \tau_{2}\right) \in(0,1)^{2}$. Its modulus squared $\left|\mathfrak{R}^{j_{1}, j_{2}}\left(\omega ; \tau_{1}, \tau_{2}\right)\right|^{2}$ is referred to as the quantile coherence kernel of $\left(X_{t, j_{1}}\right)_{t \in \mathbb{Z}}$ and $\left(X_{t, j_{2}}\right)_{t \in \mathbb{Z}}$. Note the important fact that $\mathfrak{R}^{j_{1}, j_{2}}\left(\omega ; \tau_{1}, \tau_{2}\right)$ is undefined when $\left(\tau_{1}, \tau_{2}\right)$ is on the boundary of $[0,1]^{2}$, which is due to the fact that $\operatorname{Var} \mathrm{d} Z_{j}^{\tau}(\omega)=0$ if $\tau \in\{0,1\}$. By Cauchy-Schwarz inequality, we further observe that the range of possible values is limited to $\mathfrak{R}^{j_{1}, j_{2}}\left(\omega ; \tau_{1}, \tau_{2}\right) \in\{z \in \mathbb{C}:|z| \leq 1\}$ and 


\begin{tabular}{|c|c|}
\hline Name & Symbol \\
\hline $\begin{array}{l}\text { quantile cospectrum of }\left(X_{t, j_{1}}\right)_{t \in \mathbb{Z}} \text { and }\left(X_{t, j_{2}}\right)_{t \in \mathbb{Z}} \\
\text { quantile quadrature spectrum of }\left(X_{t, j_{1}}\right)_{t \in \mathbb{Z}} \text { and }\left(X_{t, j_{2}}\right)_{t \in \mathbb{Z}} \\
\text { quantile amplitude spectrum of }\left(X_{t, j_{1}}\right)_{t \in \mathbb{Z}} \text { and }\left(X_{t, j_{2}}\right)_{t \in \mathbb{Z}} \\
\text { quantile phase spectrum of }\left(X_{t, j_{1}}\right)_{t \in \mathbb{Z}} \text { and }\left(X_{t, j_{2}}\right)_{t \in \mathbb{Z}} \\
\text { quantile coherency of of }\left(X_{t, j_{1}}\right)_{t \in \mathbb{Z}} \text { and }\left(X_{t, j_{2}}\right)_{t \in \mathbb{Z}} \\
\text { quantile coherence of of }\left(X_{t, j_{1}}\right)_{t \in \mathbb{Z}} \text { and }\left(X_{t, j_{2}}\right)_{t \in \mathbb{Z}}\end{array}$ & $\begin{array}{l}\Re \mathfrak{f}^{j_{1}, j_{2}}\left(\omega ; \tau_{1}, \tau_{2}\right) \\
-\Im^{\mathfrak{f}_{1}, j_{2}}\left(\omega ; \tau_{1}, \tau_{2}\right) \\
\left|\mathfrak{f}^{j_{1}, j_{2}}\left(\omega ; \tau_{1}, \tau_{2}\right)\right| \\
\arg \left(\mathfrak{f}_{j_{1}, j_{2}}\left(\omega ; \tau_{1}, \tau_{2}\right)\right) \\
\mathfrak{R}^{j_{1}, j_{2}}\left(\omega ; \tau_{1}, \tau_{2}\right) \\
\left|\mathfrak{R}^{j_{1}, j_{2}}\left(\omega ; \tau_{1}, \tau_{2}\right)\right|^{2}\end{array}$ \\
\hline
\end{tabular}

Table 1: Spectral quantities related to the quantile cross-spectral density kernel $\mathfrak{f}^{j_{1}, j_{2}}\left(\omega ; \tau_{1}, \tau_{2}\right)$ of $\left(X_{t, j_{1}}\right)_{t \in \mathbb{Z}}$ and $\left(X_{t, j_{2}}\right)_{t \in \mathbb{Z}}$, as defined in Section 2.2 .

$\left|\mathfrak{R}^{j_{1}, j_{2}}\left(\omega ; \tau_{1}, \tau_{2}\right)\right|^{2} \in[0,1]$, respectively. A value of $\left|\mathfrak{R}^{j_{1}, j_{2}}\left(\omega ; \tau_{1}, \tau_{2}\right)\right|^{2}$ close to 1 indicates a strong (linear) relationship between $\mathrm{d} Z_{j_{1}}^{\tau_{1}}(\omega)$ and $\mathrm{d} Z_{j_{2}}^{\tau_{2}}(\omega)$. Note that, as $\left(\tau_{1}, \tau_{2}\right)$ approaches the border of the unit square, the quantile cross-spectral density vanishes. Therefore, the quantile cross-spectral density (without the standardization) is not well suited to measure dependence of extremes. Implicitly, we take advantage of the fact that the quantile cross-spectral density and quantile spectral densities vanish at the same rate and therefore the quotient yields a meaningful quantity when the quantile levels $\left(\tau_{1}, \tau_{2}\right)$ approaches the border of the unit square.

The quantile coherency kernel and quantile coherence kernel contain very valuable information about the joint dynamics of the time series $\left(X_{t, j_{1}}\right)_{t \in \mathbb{Z}}$ and $\left(X_{t, j_{2}}\right)_{t \in \mathbb{Z}}$. In contrast to the traditional case, where coherency and coherence will always equal one if $j_{1}=j_{2}=: j$, the quantile-based versions of these quantities are capable of delivering valuable information about one single component of $\left(\boldsymbol{X}_{t}\right)_{t \in \mathbb{Z}}$ as well. More precisely, quantile coherency and quantile coherence then quantify the joint dynamics of ( $I\left\{X_{t, j} \leq\right.$ $\left.\left.q_{j}\left(\tau_{1}\right)\right\}\right)_{t \in \mathbb{Z}}$ and $\left(I\left\{X_{t, j} \leq q_{j}\left(\tau_{2}\right)\right\}\right)_{t \in \mathbb{Z}}$.

Note that all the quantities defined above are complex-valued, $2 \pi$-periodic as a function of the variable $\omega$, and Hermitian in the sense that we have

$$
\overline{\mathfrak{f}^{j_{1}, j_{2}}\left(\omega ; \tau_{1}, \tau_{2}\right)}=\mathfrak{f}^{j_{1}, j_{2}}\left(-\omega ; \tau_{1}, \tau_{2}\right)=\mathfrak{f}^{j_{2}, j_{1}}\left(\omega ; \tau_{2}, \tau_{1}\right)=\mathfrak{f}^{j_{2}, j_{1}}\left(2 \pi+\omega ; \tau_{2}, \tau_{1}\right) .
$$

Similar relations hold for quantile coherency and quantile coherence.

For the readers convenience, a list of the quantities and symbols introduced in this section is provided in Table 1 .

\subsection{Relation between quantile and traditional spectral quanti- ties}

When applying the proposed quantities, it is important to proceed with care when relating them to the traditional correlation and coherency measures. In this section we examine the case of a weakly stationary, multivariate process, where the proposed, quantile-based quantities and their traditional counterparts are directly related. The aim of the discussion is twofold. On one hand it provides assistance in how to interpret the quantile spectral quantities when the model is known to be Gaussian. On the other hand, and 
more importantly, it provides additional insight in how the traditional quantities break down when the serial dependency structure is not completely specified by the second moments.

We start by the discussion of the general case, where the process under consideration is assumed to be stationary, but needs not to be Gaussian. We will state conditions under which the traditional spectra (i.e., the matrix of spectral densities and cross-spectral densities) uniquely determines the quantile spectra (i.e., the matrix of quantile spectral densities and cross-spectral densities). In the end of this section we will discuss three examples of bivariate, stationary Gaussian processes and explain how the traditional coherency and the quantile coherency are related.

Denote by $c:=\left\{c_{k}^{j_{1}, j_{2}}: j_{1}, j_{2} \in\{1, \ldots, d\}, k \in \mathbb{Z}\right\} . c_{k}^{j_{1} j_{2}}:=\operatorname{Cov}\left(X_{t+k, j_{1}}, X_{t, j_{2}}\right)$, the family of auto- and cross-covariances. We will also refer to them as the second moment features of the process. We assume that $\left(\left|c_{k}^{j_{1}, j_{2}}\right|\right)_{k \in \mathbb{Z}}$ is summable, such that the traditional spectra $f^{j_{1}, j_{2}}(\omega):=(2 \pi)^{-1} \sum_{k \in \mathbb{Z}} c_{k}^{j_{1}, j_{2}} \mathrm{e}^{-\mathrm{i} k \omega}$ exist. Because of the relation $c_{k}^{j_{1}, j_{2}}=\int_{-\pi}^{\pi} f^{j_{1}, j_{2}}(\omega) \mathrm{e}^{\mathrm{i} k \omega} \mathrm{d} \omega$ we will equivalently refer to $\boldsymbol{f}(\omega):=\left(f^{j_{1}, j_{2}}(\omega)\right)_{j_{1}, j_{2}=1, \ldots, d}$ as the second moment features of the process.

We now state conditions under which the traditional spectra uniquely determine the quantile spectra. Assume that the marginal distribution of $X_{t, j}(j \in\{1, \ldots, d\})$, which we denote by $F_{j}$, does not depend on $t$ and is continuous. Further, the joint distribution of $\left(F_{j_{1}}\left(X_{t+k, j_{1}}\right), F_{j_{2}}\left(X_{t, j_{2}}\right)\right), j_{1}, j_{2} \in\{1, \ldots, d\}$, i. e. the copula of the pair $\left(X_{t+k, j_{1}}, X_{t, j_{2}}\right)$, shall depend only on $k$, but not on $t$, and be uniquely specified by the second moment features of the process. More precisely, we assume the existence of functions $C_{k}^{j_{1}, j_{2}}$, such that

$$
C_{k}^{j_{1}, j_{2}}\left(\tau_{1}, \tau_{2} ; \boldsymbol{c}\right)=\mathbb{P}\left(F_{j_{1}}\left(X_{t+k, j_{1}}\right) \leq \tau_{1}, F_{j_{2}}\left(X_{t, j_{2}}\right) \leq \tau_{2}\right) .
$$

Obviously, $\mathfrak{f}^{j_{1}, j_{2}}\left(\omega ; \tau_{1}, \tau_{2}\right)$ is then, if it exists, uniquely determined by $\boldsymbol{c}$ [note (2) and the fact that $\left.\gamma_{k}^{j_{1}, j_{2}}\left(\tau_{1}, \tau_{2}\right)=C_{k}^{j_{1}, j_{2}}\left(\tau_{1}, \tau_{2} ; \boldsymbol{c}\right)-\tau_{1} \tau_{2}\right]$.

In the case of stationary Gaussian processes the assumptions sufficient for the quantile spectra to be uniquely identified by the traditional spectra hold with

$$
C_{k}^{j_{1}, j_{2}}\left(\tau_{1}, \tau_{2} ; \boldsymbol{c}\right):=C^{\text {Gauss }}\left(\tau_{1}, \tau_{2} ; c_{k}^{j_{1}, j_{2}}\left(c_{0}^{j_{1}, j_{1}} c_{0}^{j_{2}, j_{2}}\right)^{-1 / 2}\right),
$$

where we have denoted the Gaussian copula by $C^{\text {Gauss }}\left(\tau_{1}, \tau_{2} ; \rho\right)$.

The converse can be stated under less restrictive conditions. If the marginal distributions are both known and both possess second moments, then the quantile spectra uniquely determine the traditional spectra.

Assume now the previously described situation in which the second moment features $\boldsymbol{f}$ uniquely determine the quantile spectra, which we denote by $\boldsymbol{f}_{\boldsymbol{f}}^{j_{1}, j_{2}}\left(\omega ; \tau_{1}, \tau_{2}\right)$ to stress the fact that it is determined by $\boldsymbol{f}$. Thus, the relation between the traditional spectra and the quantile spectra is 1-to- 1 . Denote the traditional coherency by $R^{j_{1}, j_{2}}(\omega):=$ $f^{j_{1}, j_{2}}(\omega) /\left(f^{j_{1}, j_{1}}(\omega) f^{j_{2}, j_{2}}(\omega)\right)^{1 / 2}$ and observe that it is also uniquely determined by the second moment features $\boldsymbol{f}$. Because the quantile coherency is determined by the quantile spectra which is related to the second moment features $\boldsymbol{f}$, as previously explained, we have established the relation of the traditional coherency and the quantile coherency. Obviously, this relation is not necessarily 1-to-1 anymore. 
If the stationary process is from a parametric family of time series models the second moment features can be determined for each parameter. We now discuss three examples of Gaussian processes. Each example will have more complex serial dependence than the previous one. Without loss of generality we consider only bivariate examples. The first example is the one of non-degenerate Gaussian white noise. More precisely, we consider a Gaussian process $\left(X_{t, 1}, X_{t, 2}\right)_{t \in \mathbb{Z}}$, where $\operatorname{Cov}\left(X_{t, i}, X_{s, j}\right)=0$ and $\operatorname{Var}\left(X_{t, i}\right)>0$, for all $t \neq s$ and $i, j \in\{1,2\}$.

Observe that, due to the independence of $\left(X_{t, 1}, X_{t, 2}\right)$ and $\left(X_{s, 1}, X_{s, 2}\right), t \neq s$, we have $\gamma_{k}^{1,2}\left(\tau_{1}, \tau_{2}\right)=0$ for all $k \neq 0$ and $\tau_{1}, \tau_{2} \in[0,1]$. It is easy to see that

$$
\mathfrak{R}^{1,2}\left(\omega ; \tau_{1}, \tau_{2}\right)=\frac{C^{\text {Gauss }}\left(\tau_{1}, \tau_{2} ; R^{1,2}(\omega)\right)-\tau_{1} \tau_{2}}{\sqrt{\tau_{1}\left(1-\tau_{1}\right)} \sqrt{\left.\tau_{2}\left(1-\tau_{2}\right)\right)}}
$$

where $R^{1,2}(\omega)$ denotes the traditional coherency, which in this case (a bivariate i.i.d. sequence) equals $c_{0}^{1,2}\left(c_{0}^{1,1} c_{0}^{2,2}\right)^{-1 / 2}$ (for all $\omega$ ).

By employing (6), we can thus determine the quantile coherency for any given traditional coherency and fixed combination of $\tau_{1}, \tau_{2} \in(0,1)$. In the top-center part of Figure 2 this conversion is visualized for four pairs of quantile levels and any possible traditional coherency. It is important to observe the limited range of the quantile coherency. For example, there never is strong positive dependence between the $\tau_{1}$-quantile in the first component and the $\tau_{2}$-quantile in the second component when both $\tau_{1}$ and $\tau_{2}$ are close to 0 . Similarly, there never is strong negative dependence when one of the quantile levels is chosen close to 0 while the other one is chosen close to 1 . This observation is not special for the Gaussian case, but holds for any sequence of pairwise independent bivariate random variables. Bounds that correspond to the case of perfect positive or perfect negative dependence (at the level of quantiles), can be derived from the Fréchet/Hoeffding bounds for copulas: in the case of serial independence quantile coherency is bounded by

$$
\frac{\max \left\{\tau_{1}+\tau_{2}-1,0\right\}-\tau_{1} \tau_{2}}{\sqrt{\tau_{1}\left(1-\tau_{1}\right)} \sqrt{\tau_{2}\left(1-\tau_{2}\right)}} \leq \mathfrak{R}^{1,2}\left(\omega ; \tau_{1}, \tau_{2}\right) \leq \frac{\min \left\{\tau_{1}, \tau_{2}\right\}-\tau_{1} \tau_{2}}{\sqrt{\tau_{1}\left(1-\tau_{1}\right)} \sqrt{\left.\tau_{2}\left(1-\tau_{2}\right)\right)}}
$$

Note that these bounds hold for any joint distribution of $\left(X_{t, i}, X_{t, j}\right)$. In particular, the bound holds independent of the correlation.

In the top-left part of Figure 2 traditional coherencies are shown for this example. Because no serial dependence is present, all coherencies are flat lines. Their level is equal to the correlation between the two components. In the top-right part of Figure 2 the quantile coherency for the example is shown when the correlation is 0.6 (the corresponding coherency is marked with a bold line in the top-left figure). Note that for fixed $\tau_{1}$ and $\tau_{2}$ the value of the quantile coherency corresponds to the value in the top-center figure where the vertical gray line and the corresponding graph intersect. The quantile coherency in the right part does not depend on the frequency, because in this example there is no serial dependence.

In the top-center part of Figure 2 it is important to observe that for correlation 0 (i. e., when the components are independent, due to $\left(X_{t, 1}, X_{t, 2}\right)$ being uncorrelated jointly Gaussian) quantile coherency is zero at all quantile levels. 

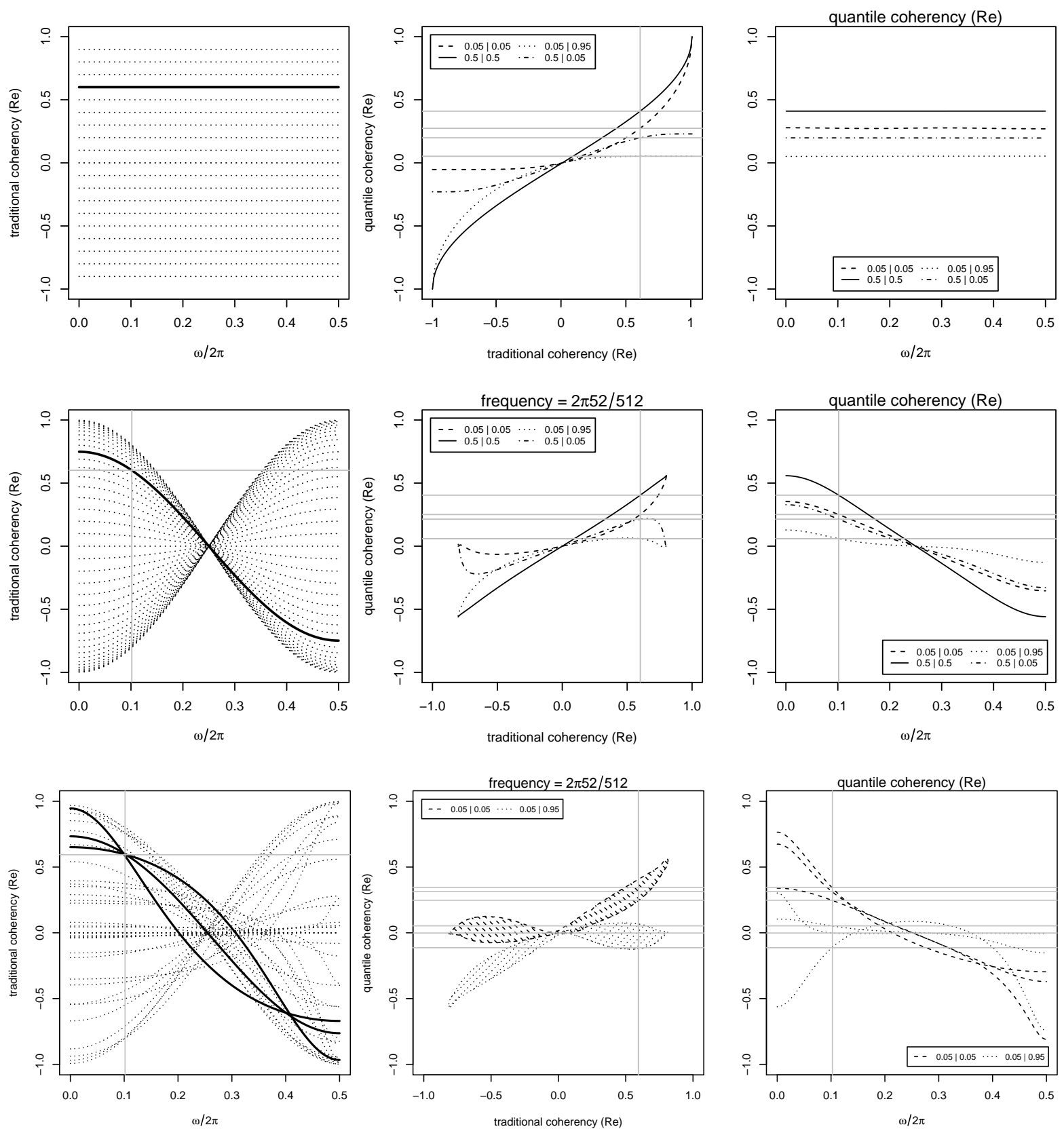

Figure 2: Each row corresponds to one of the three examples in the text. Top: Gaussian white noise. Middle: $\operatorname{VAR}(1)$ with $\boldsymbol{X}_{t}=\left(\begin{array}{cc}0 & a \\ a & 0\end{array}\right) \boldsymbol{X}_{t-1}+\varepsilon_{t},|a|<1$, where $\left(\varepsilon_{t}\right)$ is Gaussian white noise, with $\mathbb{E}\left(\varepsilon_{t} \varepsilon_{t}^{\prime}\right)=\boldsymbol{I}_{2}$. Bottom: $\operatorname{VAR}(1)$ with $\boldsymbol{X}_{t}=\left(\begin{array}{cc}b & a \\ a & b\end{array}\right) \boldsymbol{X}_{t-1}+\varepsilon_{t},|a+b|<1$, where $\left(\varepsilon_{t}\right)$ is as before. Left: traditional coherencies. For examples 1 and 2 the coherency that has the value of 0.6 at $2 \pi 51 / 512$ is shown in bold; in example 3 three such coherencies are shown. Center: Relationship between traditional coherency and quantile coherency; gray grid lines indicate how the traditional coherency of 0.6 translate to quantile coherency. In examples 2 and 3, where serial dependence is present, the cases where $\omega=2 \pi 52 / 512$ is shown. Right: quantile coherency for the example(s) where the traditional coherency is 0.6 (for $\omega=2 \pi 52 / 512$ in examples 2 and 3 ). 
In the next two examples we stay in the Gaussian framework, but introduce serial dependence. Consider a bivariate, stable $\operatorname{VAR}(1)$ process $\boldsymbol{X}_{t}=\left(X_{t, 1}, X_{t, 2}\right)^{\prime}, t \in \mathbb{Z}$, fulfilling the difference equation

$$
\boldsymbol{X}_{t}=\boldsymbol{A} \boldsymbol{X}_{t-1}+\varepsilon_{t}
$$

with parameter $\boldsymbol{A} \in \mathbb{R}^{2 \times 2}$ and i.i.d., centered, bivariate, jointly normally distributed innovations $\varepsilon_{t}$ with unit variance $\mathbb{E}\left(\varepsilon_{t} \varepsilon_{t}^{\prime}\right)=\boldsymbol{I}_{2}$.

In our second example serial dependence is introduced, by relating each component to the lagged other component in the regression equation. In other words, we consider model (7) where the matrix $\boldsymbol{A}$ has diagonal elements equal to 0 and some value $a$ on the off-diagonal. Assuming $|a|<1$ yields a stable process. As described earlier, the traditional spectral density matrix, which in this example is of the form

$$
\boldsymbol{f}(\omega):=(2 \pi)^{-1}\left(\boldsymbol{I}_{2}-\left(\begin{array}{cc}
0 & a \\
a & 0
\end{array}\right) \mathrm{e}^{-i \omega}\right)^{-1}\left(\boldsymbol{I}_{2}-\left(\begin{array}{cc}
0 & a \\
a & 0
\end{array}\right) \mathrm{e}^{i \omega}\right)^{-1},|a|<1
$$

uniquely determines the traditional coherency and, because of the Gaussian innovations, also the quantile coherency.

In the middle-left plot of Figure 2 the traditional coherencies for this model are shown when $a$ takes different values. If we now fix a frequency $[\neq \pi / 4]$, then the value of the traditional coherency for this frequency uniquely determines the value of $a$. In Figure 2 we have marked the frequency of $\omega=2 \pi 52 / 512$ and coherency value of 0.6 by gray lines and printed the corresponding coherency (as a function of $\omega$ ) in bold. Note that of the many pictured coherencies [one for each $a \in(-1,1)$ ] only one has the value of 0.6 at this frequency. In the center plot of the middle row we show the relation between the traditional coherency and quantile coherency for the considered model. For four combinations of quantile levels and all values of $a \in(-1,1)$ the corresponding traditional coherencies and quantile coherencies are shown. It is important to observe that the relation is shown only for one frequency $[\omega=2 \pi 52 / 512]$. We observe that the range of values for the quantile coherency is limited and that the range depends on the combination of quantile levels and on the frequency. While this is quite similar to the first example where quantile coherency had to be bounded due to the Fréchet/Hoeffding bounds, we here also observe (for this particular model and frequency) that the range of values for the traditional coherency is limited. This fact is also apparent in the middleleft plot. To relate the traditional and quantile coherency at this particular frequency, one can, using the center-middle plot, proceed as in the first example. For a given frequency choose a valid traditional coherency (x-axis of the middle-center plot) and combination of quantile levels (one of the lines in the plot) and then determine the value for the quantile coherency (depicted in the right plot). Note that (in this example), for a given frequency and combination of quantile levels the relation is still a function of the traditional coherency, but fails to be injective.

In our final example we consider the Gaussian VAR(1) model (7) where we now allow for an additional degree of freedom, by letting the matrix $\boldsymbol{A}$ be of the form where the diagonal elements both are equal to $b$ and keep the value $a$ on the off-diagonal as before. Thus, compared to the previous example, where $b=0$ was required, each component 
now may also depend on its own lagged value. It is easy to see that $|a+b|<1$ yields a stable process. In this case the tradtional spectral density matrix is of the form

$$
\boldsymbol{f}(\omega):=(2 \pi)^{-1}\left(\boldsymbol{I}_{2}-\left(\begin{array}{ll}
b & a \\
a & b
\end{array}\right) \mathrm{e}^{-i \omega}\right)^{-1}\left(\boldsymbol{I}_{2}-\left(\begin{array}{ll}
b & a \\
a & b
\end{array}\right) \mathrm{e}^{i \omega}\right)^{-1},|a+b|<1 .
$$

In the bottom-left part of Figure 2 a collection of traditional coherencies (as functions of $\omega$ ) is shown. Due to the extra degree of freedom in the model the variety of shapes increased dramatically. In particular, for a given frequency, the value of the traditional coherency does not uniquely specify the model parameter any more. We have marked three coherencies (as functions of $\omega$ ) that have value 0.6 at $\omega=2 \pi 52 / 512$ in bold to stress this fact. The corresponding processes have (for a fixed combination of quantile levels) different values of quantile coherency at this frequency. This fact can be seen from the bottom-center part of Figure 2, where the relation between traditional and quantile coherency is depicted for the frequency fixed and two combinations of quantile levels are shown in black and gray. Note the important fact that the relation (for fixed frequency) is not a function of the traditional coherency any more. The bottom-right part of the figure shows the quantile coherency curves (as a function of $\omega$ ) for the three model parameters (shown in bold in the bottom-left part of the figure) and the two combination of quantile levels. It is clearly visible that even though, for the particular fixed frequency, the traditional coherency coincide, the value and shape of the quantile coherency can be very different depending on the underlying process. This third example illustrated how a frequency-by-frequency comparison of the traditional coherency with its quantile-based counterpart may fail, even when the process is quite simple.

We have seen, from the theoretical discussion in the beginning of this section, that for Gaussian processes, when the marginal distributions are fixed, a relation between the traditional spectra and the quantile spectra exists. This relation is a 1-to-1 relation between the quantities as functions of frequency (and quantile levels). The three examples have illustrated that a comparison on a frequency-by-frequency basis may be possible in special cases but does not hold in general.

In conclusion we therefore advise to see the quantile cross-spectral density as a measure for dependence on its own, as the quantile-based quantities focus on more general types of dependence. We further point out that quantile coherency may be used in examples where the conditions that make a relation possible are fulfilled, but also, for example, to analyze the dependence in the quantile vector autoregressive (QVAR) processes, described in Section 4. The QVAR processes possess more complicated dynamics, which cannot be described only by the second order moment features.

\subsection{Estimation}

For the univariate case $\left(j_{1}=j_{2}\right)$, different approaches to consistent estimation were considered. $\mathrm{Li}(2008)$ proposed an estimator, based on least absolute deviation regression, for the special case where $\tau_{1}=\tau_{2}=0.5$. He later $(\mathrm{Li}, 2012)$ generalized the estimator, using quantile regression, to the case where $\tau_{1}=\tau_{2} \in(0,1)$. The general case, in which

the quantities can be related to the copulas of pairs, was first considered by Dette et al. 
(2015). These authors also introduce a rank-based version of the quantile regressiontype estimator for this case. A different approach to estimation was taken by Hagemann (2013). Again for the special cases where $j_{1}=j_{2}$ and $\tau_{1}=\tau_{2} \in(0,1)$, Hagemann (2013) proposed a version of the traditional $L^{2}$-periodogram where the observations are replaced with $I\left\{\hat{F}_{n, j}\left(X_{t, j}\right) \leq \tau\right\}=I\left\{R_{n ; t, j} \leq n \tau\right\}$, where $\hat{F}_{n, j}(x):=n^{-1} \sum_{t=0}^{n-1} I\left\{X_{t, j} \leq x\right\}$ denotes the empirical distribution function of $X_{t, j}$ and $R_{n ; t, j}$ denotes the (maximum) rank of $X_{t, j}$ among $X_{0, j}, \ldots, X_{n-1, j}$. Kley et al. (2015) generalized this estimator, in the spirit of Dette et al. (2015), by considering cross-periodograms for arbitrary couples $\left(\tau_{1}, \tau_{2}\right) \in[0,1]^{2}$, and proved that it converges, as a stochastic process, to a complex-valued Gaussian limit. An estimator defined in analogy to the traditional lag-window estimator was analyzed by Birr et al. (2015) in the context of non-stationary time series.

In this paper we define, in the spirit of Hagemann (2013) and Kley et al. (2015), the estimator for the quantile cross-spectral density as follows. The collection

$$
I_{n, R}^{j_{1}, j_{2}}\left(\omega ; \tau_{1}, \tau_{2}\right):=\frac{1}{2 \pi n} d_{n, R}^{j_{1}}\left(\omega ; \tau_{1}\right) d_{n, R}^{j_{2}}\left(-\omega ; \tau_{2}\right)
$$

$j_{1}, j_{2}=1, \ldots, d, \omega \in \mathbb{R},\left(\tau_{1}, \tau_{2}\right) \in[0,1]^{2}$, will be called the rank-based copula crossperiodograms, shortly, the CCR-periodograms, where

$$
d_{n, R}^{j}(\omega ; \tau):=\sum_{t=0}^{n-1} I\left\{\hat{F}_{n, j}\left(X_{t, j}\right) \leq \tau\right\} \mathrm{e}^{-\mathrm{i} \omega t}=\sum_{t=0}^{n-1} I\left\{R_{n ; t, j} \leq n \tau\right\} \mathrm{e}^{-\mathrm{i} \omega t},
$$

$j=1, \ldots, d, \omega \in \mathbb{R}, \tau \in[0,1]$. We will denote the matrix of CCR-periodograms by

$$
\boldsymbol{I}_{n, R}\left(\omega ; \tau_{1}, \tau_{2}\right):=\left(I_{n, R}^{j_{1}, j_{2}}\left(\omega ; \tau_{1}, \tau_{2}\right)\right)_{j_{1}, j_{2}=1, \ldots, d}
$$

From the univariate case it is already known (cf. Proposition 3.4 in Kley et al. (2015)) that the CCR-periodograms fail to estimate $\mathfrak{f}^{j_{1}, j_{2}}\left(\omega ; \tau_{1}, \tau_{2}\right)$ consistently. Consistency can be achieved by smoothing $I_{n, R}^{j_{1}, j_{2}}\left(\omega ; \tau_{1}, \tau_{2}\right)$ across frequencies. More precisely, in this paper, we will consider

$$
\hat{G}_{n, R}^{j_{1}, j_{2}}\left(\omega ; \tau_{1}, \tau_{2}\right):=\frac{2 \pi}{n} \sum_{s=1}^{n-1} W_{n}(\omega-2 \pi s / n) I_{n, R}^{j_{1}, j_{2}}\left(2 \pi s / n, \tau_{1}, \tau_{2}\right),
$$

where $W_{n}$ denotes a sequence of weight functions, precisely to be defined in Section 3 .

We will denote the matrix of smoothed CCR-periodograms by

$$
\hat{\boldsymbol{G}}_{n, R}\left(\omega ; \tau_{1}, \tau_{2}\right):=\left(\hat{G}_{n, R}^{j_{1}, j_{2}}\left(\omega ; \tau_{1}, \tau_{2}\right)\right)_{j_{1}, j_{2}=1, \ldots, d} .
$$

Estimators for the quantile cospectrum, quantile quadrature spectrum, quantile amplitude spectrum, quantile phase spectrum, quantile coherency and quantile coherence are then given by $\Re \hat{G}_{n, R}^{j_{1}, j_{2}}\left(\omega ; \tau_{1}, \tau_{2}\right),-\Im \hat{G}_{n, R}^{j_{1}, j_{2}}\left(\omega ; \tau_{1}, \tau_{2}\right),\left|\hat{G}_{n, R}^{j_{1}, j_{2}}\left(\omega ; \tau_{1}, \tau_{2}\right)\right|, \arg \left(\hat{G}_{n, R}^{j_{1}, j_{2}}\left(\omega ; \tau_{1}, \tau_{2}\right)\right)$,

$$
\hat{\mathfrak{R}}_{n, R}^{j_{1}, j_{2}}\left(\omega ; \tau_{1}, \tau_{2}\right):=\frac{\hat{G}_{n, R}^{j_{1}, j_{2}}\left(\omega ; \tau_{1}, \tau_{2}\right)}{\left(\hat{G}_{n, R}^{j_{1}, j_{1}}\left(\omega ; \tau_{1}, \tau_{1}\right) \hat{G}_{n, R}^{j_{2}, j_{2}}\left(\omega ; \tau_{2}, \tau_{2}\right)\right)^{1 / 2}}
$$

and $\left|\hat{\mathfrak{R}}_{n, R}^{j_{1}, j_{2}}\left(\omega ; \tau_{1}, \tau_{2}\right)\right|^{2}$, respectively. 


\section{Asymptotic properties of the proposed estimators}

To derive the asymptotic properties of the estimators defined in Section 2.4 some assumptions on the underlying process $\left(\boldsymbol{X}_{t}\right)_{t \in \mathbb{Z}}$ and the weighting functions $W_{n}$ need to be made.

Recall (cf. Brillinger (1975), p. 19) that the $r$ th order joint cumulant $\operatorname{cum}\left(Z_{1}, \ldots, Z_{r}\right)$ of the random vector $\left(Z_{1}, \ldots, Z_{r}\right)$ is defined as

$$
\operatorname{cum}\left(Z_{1}, \ldots, Z_{r}\right):=\sum_{\left\{\nu_{1}, \ldots, \nu_{p}\right\}}(-1)^{p-1}(p-1) !\left(\mathbb{E} \prod_{j \in \nu_{1}} Z_{j}\right) \cdots\left(\mathbb{E} \prod_{j \in \nu_{p}} Z_{j}\right)
$$

with summation extending over all partitions $\left\{\nu_{1}, \ldots, \nu_{p}\right\}, p=1, \ldots, r$, of $\{1, \ldots, r\}$.

Regarding the range of dependence of $\left(\boldsymbol{X}_{t}\right)_{t \in \mathbb{Z}}$ we make the following assumption,

(C) There exist constants $\rho \in(0,1)$ and $K<\infty$ such that, for arbitrary intervals $A_{1}, \ldots, A_{p} \subset \mathbb{R}$, arbitrary indices $j_{1}, \ldots, j_{p} \in\{1, \ldots, d\}$ and times $t_{1}, \ldots, t_{p} \in \mathbb{Z}$,

$$
\left|\operatorname{cum}\left(I\left\{X_{t_{1}, j_{1}} \in A_{1}\right\}, \ldots, I\left\{X_{t_{p}, j_{p}} \in A_{p}\right\}\right)\right| \leq K \rho^{\max _{i, j}\left|t_{i}-t_{j}\right|} .
$$

Note that this assumption is a generalization of the assumption made in Kley et al. (2015) to multivariate processes. It is important to observe that Assumption (C) does not require the existence of any moments, which is in sharp contrast to the classical assumptions, where moments up to the order of the respective cumulants have to exist. Furthermore, note that the sets $A_{j}$ in $(12)$ are not required to be general Borel sets as in classical mixing assumptions.

The relation of assumption (C) to the classical $\alpha$-mixing assumption is summarized in form of the following proposition.

Proposition 3.1. Assume that the process $\left(\boldsymbol{X}_{t}\right)_{t \in \mathbb{Z}}$ is strictly stationary and exponentially $\alpha$-mixing, i.e.,

$$
\alpha(n):=\sup _{\substack{A \in \sigma\left(\boldsymbol{X}_{0}, \boldsymbol{X}_{-1}, \ldots\right) \\ B \in \sigma\left(\boldsymbol{X}_{n}, \boldsymbol{X}_{n+1}, \ldots\right)}}|\mathbb{P}(A \cap B)-\mathbb{P}(A) \mathbb{P}(B)| \leq K \kappa^{n}, n \in \mathbb{N}
$$

for some $K<\infty$ and $\kappa \in(0,1)$. Then Assumption $(C)$ holds.

Proof of Proposition 3.1. The proof is almost identical to the proof of Proposition 3.1 in Kley et al. (2015) and is therefore omitted.

Proposition 3.1 implies that Assumption (C) will hold for a wide range of popular, linear and nonlinear, multivariate and univariate processes that are known to be $\alpha$ - or $\beta$-mixing at an exponential rate. Examples of such processes (possibly, under mild additional assumptions) include the traditional (V)ARMA, general nonlinear scalar ARCH, vector-ARCH(1), threshold ARCH, and exponential ARCH processes [cf. Liebscher (2005)], GARCH $(p, q)$ processes with moments [cf. Boussama (1998)] and GARCH $(1,1)$ processes with no assumptions regarding the moments [cf. Francq and Zakoïan (2006)], generalized polynomial random coefficient vector autoregressive processes, and a family of generalized hidden Markov processes [cf. Carrasco and Chen (2002)] which include stochastic volatility models. 
Denote by $\rightsquigarrow$ the weak convergence in the sense of Hoffman-Jørgensen (cf. Chapter 1 of van der Vaart and Wellner (1996)). The estimators under consideration take values in the space of (element-wise) bounded functions $[0,1]^{2} \rightarrow \mathbb{C}^{d \times d}$, which we denote by $\ell_{\mathbb{C}^{d \times d}}^{\infty}\left([0,1]^{2}\right)$. While results in empirical process theory are typically stated for spaces of real-valued, bounded functions, these results transfer immediately by identifying $\ell_{\mathbb{C}^{d \times d}}^{\infty}\left([0,1]^{2}\right)$ with the product space $\ell^{\infty}\left([0,1]^{2}\right)^{2 d^{2}}$. Note that the space $\ell_{\mathbb{C}^{d \times d}}^{\infty}\left([0,1]^{2}\right)$ is constructed along the same lines as the space $\ell_{\mathbb{C}}^{\infty}\left([0,1]^{2}\right)$ in Kley et al. (2015).

We are now ready to state the first result on the asymptotic properties of the CCRperiodogram $\boldsymbol{I}_{n, R}\left(\omega ; \tau_{1}, \tau_{2}\right)$ defined in (8) and (9)

Proposition 3.2. Assume that $\left(\boldsymbol{X}_{t}\right)_{t \in \mathbb{Z}}$ is strictly stationary and satisfies Assumption $(C)$. Further assume that the marginal distributions $F_{j}, j=1, \ldots, d$ are continuous. Then, for every fixed $\omega \neq 0 \bmod 2 \pi$,

$$
\left(\boldsymbol{I}_{n, R}\left(\omega ; \tau_{1}, \tau_{2}\right)\right)_{\left(\tau_{1}, \tau_{2}\right) \in[0,1]^{2}} \rightsquigarrow\left(\mathbb{I}\left(\omega ; \tau_{1}, \tau_{2}\right)\right)_{\left(\tau_{1}, \tau_{2}\right) \in[0,1]^{2}} \text { in } \ell_{\mathbb{C}^{d \times d}}^{\infty}\left([0,1]^{2}\right) .
$$

The $\mathbb{C}^{d \times d}$-valued limiting processes $\mathbb{I}$, indexed by $\left(\tau_{1}, \tau_{2}\right) \in[0,1]^{2}$, is of the form

$$
\mathbb{I}\left(\omega ; \tau_{1}, \tau_{2}\right)=\frac{1}{2 \pi} \mathbb{D}\left(\omega ; \tau_{1}\right) \overline{\mathbb{D}\left(\omega ; \tau_{2}\right)^{\prime}}
$$

where $\mathbb{D}(\omega ; \tau)=\left(\mathbb{D}^{j}(\omega ; \tau)\right)_{j=1, \ldots, d}, \tau \in[0,1], \omega \in \mathbb{R}$ is a centered, $\mathbb{C}^{d}$-valued Gaussian processes with covariance structure of the following form

$$
\operatorname{Cov}\left(\mathbb{D}^{j_{1}}\left(\omega ; \tau_{1}\right), \mathbb{D}^{j_{2}}\left(\omega ; \tau_{2}\right)\right)=2 \pi \mathfrak{f}^{j_{1}, j_{2}}\left(\omega ; \tau_{1}, \tau_{2}\right)
$$

Moreover, $\mathbb{D}(\omega ; \tau)=\overline{\mathbb{D}(-\omega ; \tau)}=\mathbb{D}(\omega+2 \pi ; \tau)$, and the family $\{\mathbb{D}(\omega ; \cdot): \omega \in[0, \pi]\}$ is a collection of independent processes. In particular, the weak convergence (14) holds jointly for any finite fixed collection of frequencies $\omega$.

Proof. Deferred to the Appendix (Section 7.2).

For $\omega=0 \bmod 2 \pi$ the asymptotic behavior of the CCR-periodogram is as follows: we have $d_{n, R}^{j}(0 ; \tau)=n \tau+o_{P}\left(n^{1 / 2}\right)$, where the exact form of the remainder term depends on the number of ties in $X_{j, 0}, \ldots, X_{j, n-1}$. Therefore, under the assumptions of Proposition 3.2, we have $\boldsymbol{I}_{n, R}\left(0 ; \tau_{1}, \tau_{2}\right)=n(2 \pi)^{-1} \tau_{1} \tau_{2} 1_{d} 1_{d}^{\prime}+o_{P}(1)$, where $1_{d}:=(1, \ldots, 1)^{\prime} \in \mathbb{R}^{d}$.

In order to establish the convergence of the smoothed CCR-periodogram process, defined in (10) and (11), an assumption regarding the weights $W_{n}$ in $(10)$ is in order. For a sequence of scaling parameters $b_{n}>0, n=1,2, \ldots$, that satisfy $b_{n} \rightarrow 0$ and $n b_{n} \rightarrow \infty$, as $n \rightarrow \infty$, we define

$$
W_{n}(u):=\sum_{j=-\infty}^{\infty} b_{n}^{-1} W\left(b_{n}^{-1}[u+2 \pi j]\right)
$$

and assume that the function $W$ satisfies

(W) The weight function $W$ is real-valued, even, has support $[-\pi, \pi]$, bounded variation, and satisfies $\int_{-\pi}^{\pi} W(u) \mathrm{d} u=1$. 
Assumption (W) is quite standard in classical time series analysis [see, for example, p. 147 of Brillinger (1975)].

We now are ready to state the first main result of this paper, where the uncertainty in estimating $\mathfrak{f}\left(\omega ; \tau_{1}, \tau_{2}\right)$ by $\boldsymbol{G}_{n, R}\left(\omega ; \tau_{1}, \tau_{2}\right)$ is asymptotically described.

Theorem 3.3. Let Assumptions $(C)$ and $(W)$ hold. Assume that the distribution functions $F_{j}, j=1, \ldots, d$ are continuous and that constants $\kappa>0$ and $k \in \mathbb{N}$ exist, such that $b_{n}=o\left(n^{-1 /(2 k+1)}\right)$ and $b_{n} n^{1-\kappa} \rightarrow \infty$. Then, for any fixed $\omega \in \mathbb{R}$, the process

$$
\mathbb{G}_{n}(\omega ; \cdot, \cdot):=\sqrt{n b_{n}}\left(\hat{\boldsymbol{G}}_{n, R}\left(\omega ; \tau_{1}, \tau_{2}\right)-\mathfrak{f}\left(\omega ; \tau_{1}, \tau_{2}\right)-\boldsymbol{B}_{n}^{(k)}\left(\omega ; \tau_{1}, \tau_{2}\right)\right)_{\tau_{1}, \tau_{2} \in[0,1]}
$$

satisfies

$$
\mathbb{G}_{n}(\omega ; \cdot, \cdot) \rightsquigarrow \mathbb{H}(\omega ; \cdot, \cdot) \text { in } \ell_{\mathbb{C}^{d \times d}}^{\infty}\left([0,1]^{2}\right),
$$

where the elements of the bias matrix $\boldsymbol{B}_{n}^{(k)}$ are given by

$$
\left\{\boldsymbol{B}_{n}^{(k)}\left(\omega ; \tau_{1}, \tau_{2}\right)\right\}_{j_{1}, j_{2}}:=\sum_{\ell=2}^{k} \frac{b_{n}^{\ell}}{\ell !} \int_{-\pi}^{\pi} v^{\ell} W(v) d v \frac{\mathrm{d}^{\ell}}{\mathrm{d} \omega^{\ell}} \mathfrak{f}^{j_{1}, j_{2}}\left(\omega ; \tau_{1}, \tau_{2}\right)
$$

and $\mathfrak{f}^{j_{1}, j_{2}}\left(\omega ; \tau_{1}, \tau_{2}\right)$ is defined in (2). The process $\mathbb{H}(\omega ; \cdot, \cdot):=\left(\mathbb{H}^{j_{1}, j_{2}}(\omega ; \cdot, \cdot)\right)_{j_{1}, j_{2}=1, \ldots, d}$ in (15) is a centered, $\mathbb{C}^{d \times d}$-valued Gaussian process characterized by

$$
\begin{aligned}
& \operatorname{Cov}\left(\mathbb{H}^{j_{1}, j_{2}}\left(\omega ; u_{1}, v_{1}\right), \mathbb{H}^{k_{1}, k_{2}}\left(\lambda ; u_{2}, v_{2}\right)\right) \\
&=2 \pi\left(\int_{-\pi}^{\pi} W^{2}(\alpha) \mathrm{d} \alpha\right)\left(\mathfrak{f}^{j_{1}, k_{1}}\left(\omega ; u_{1}, u_{2}\right) \mathfrak{f}^{j_{2}, k_{2}}\left(-\omega ; v_{1}, v_{2}\right) \eta(\omega-\lambda)\right. \\
&\left.\quad+\mathfrak{f}^{j_{1}, k_{2}}\left(\omega ; u_{1}, v_{2}\right) \mathfrak{f}^{j_{2}, k_{1}}\left(-\omega ; v_{1}, u_{2}\right) \eta(\omega+\lambda)\right),
\end{aligned}
$$

where $\eta(x):=I\{x=0(\bmod 2 \pi)\}$ [cf. [Brillinger, 1975, p. 148)] is the $2 \pi$-periodic extension of Kronecker's delta function. The family $\{\mathbb{H}(\omega ; \cdot, \cdot), \omega \in[0, \pi]\}$ is a collection of independent processes and $\mathbb{H}\left(\omega ; \tau_{1}, \tau_{2}\right)=\overline{\mathbb{H}}\left(-\omega ; \tau_{1}, \tau_{2}\right)=\mathbb{H}\left(\omega+2 \pi ; \tau_{1}, \tau_{2}\right)$.

Proof. Deferred to the Appendix (Section 7.3).

A few remarks on the result are in order. In sharp contrast to classical spectral analysis, where higher-order moments are required to obtain smoothness of the spectral density [cf. Brillinger (1975), p. 27], Assumption (C) guarantees that the quantile crossspectral density is an analytical function of $\omega$ [cf. Lemma 7.6]. Hence, the $k$ th derivative of $\omega \mapsto \mathfrak{f}^{j_{1}, j_{2}}\left(\omega ; \tau_{1}, \tau_{2}\right)$ in $(16)$ exists without further assumptions.

Assume that $W$, for some $p$, satisfies $\int_{-\pi}^{\pi} v^{j} W(v) \mathrm{d} v=0$, for $j<p$, and $0<$ $\int_{-\pi}^{\pi} v^{p} W(v) \mathrm{d} v<\infty$. Such kernels are typically referred to as kernels of order $p$; the Epanechnikov kernel, for example, is of order $p=2$. Then, the bias is of order $b_{n}^{p}$. As the variance is of order $\left(n b_{n}\right)^{-1}$, the mean squared error is minimal, if $b_{n} \asymp n^{-1 /(2 p+1)}$. This optimal bandwidth fulfills the assumptions of Theorem 3.3 .

We can use Theorem 3.3 to construct asymptotically valid confidence intervals. A detailed discussion of the construction of confidence intervals is deferred to Section 7.1.1, 
The independence of the limit $\{\mathbb{H}(\omega ; \cdot, \cdot), \omega \in[0, \pi]\}$ has two important implications. On one hand, the weak convergence (15) holds jointly for any finite fixed collection of frequencies $\omega$. On the other hand, if one were to consider the smoothed CCR-periodogram as a function of the three arguments $\left(\omega, \tau_{1}, \tau_{2}\right)$, weak convergence cannot hold any more. This limitation of convergence is due to the fact that there exists no tight element in $\ell_{\mathbb{C}^{d \times d}}^{\infty}\left([0, \pi] \times[0,1]^{2}\right)$ that has the right finite-dimensional distributions, which would be required for process convergence in $\ell_{\mathbb{C}^{d \times d}}^{\infty}\left([0, \pi] \times[0,1]^{2}\right)$.

Fixing $j_{1}, j_{2}$ and $\tau_{1}, \tau_{2}$ the CCR-periodogram $\hat{G}_{n, R}^{j_{1}, j_{2}}\left(\omega ; \tau_{1}, \tau_{2}\right)$ and traditional smoothed cross-periodogram determined from the unobservable, bivariate time series

$$
\left(I\left\{F_{j_{1}}\left(X_{t, j_{1}}\right) \leq \tau_{1}\right\}, I\left\{F_{j_{1}}\left(X_{t, j_{2}}\right) \leq \tau_{2}\right\}\right), \quad t=0, \ldots, n-1,
$$

are asymptotically equivalent. Theorem 3.3 thus reveals that in the context of the estimation of the quantile cross-spectral density the estimation of the marginal distribution has no impact on the limit distribution.

We now turn our attention to the estimation of quantile coherency. A consistent estimator for the matrix of quantile coherencies

$$
\mathfrak{R}\left(\omega ; \tau_{1}, \tau_{2}\right):=\left(\mathfrak{R}^{j_{1}, j_{2}}\left(\omega ; \tau_{1}, \tau_{2}\right)\right)_{j_{1}, j_{2}=1, \ldots, d}
$$

is given by the $\mathbb{C}^{d \times d}$-valued function

$$
\hat{\mathfrak{R}}_{n, R}\left(\omega ; \tau_{1}, \tau_{2}\right):=\left(\hat{\mathfrak{R}}_{n, R}^{j_{1}, j_{2}}\left(\omega ; \tau_{1}, \tau_{2}\right)\right)_{j_{1}, j_{2}=1, \ldots, d}
$$

The second main result of this paper is about the asymptotic behavior of $\hat{\mathfrak{R}}_{n, R}\left(\omega ; \tau_{1}, \tau_{2}\right)$ as an estimator for $\mathfrak{R}\left(\omega ; \tau_{1}, \tau_{2}\right)$.

Theorem 3.4. Let the Assumptions for Theorem 3.3 hold. Furthermore, assume that for some $\varepsilon \in(0,1 / 2)$ we have

$$
\inf _{\tau \in[\varepsilon, 1-\varepsilon]} \mathfrak{f}^{j, j}(\omega ; \tau, \tau)>0, \text { for all } j=1, \ldots, d,
$$

and that $b_{n}$ satisfies

$$
\sup _{\tau_{1}, \tau_{2} \in[\varepsilon, 1-\varepsilon]}\left|\left\{\boldsymbol{B}_{n}^{(k)}\left(\omega ; \tau_{1}, \tau_{2}\right)\right\}_{j_{1}, j_{2}}\right|=o\left(\left(n b_{n}\right)^{-1 / 4}\right) \text {, for all } j_{1}, j_{2}=1, \ldots, d \text {. }
$$

Then, for any fixed $\omega \in \mathbb{R}$,

$$
\sqrt{n b_{n}}\left(\hat{\mathfrak{\Re}}_{n, R}\left(\omega ; \tau_{1}, \tau_{2}\right)-\mathfrak{R}\left(\omega ; \tau_{1}, \tau_{2}\right)-\mathfrak{B}_{n}^{(k)}\left(\omega ; \tau_{1}, \tau_{2}\right)\right)_{\left(\tau_{1}, \tau_{2}\right) \in[0,1]^{2}} \rightsquigarrow \mathbb{L}(\omega ; \cdot, \cdot),
$$

in $\ell_{\mathbb{C}^{d \times d}}^{\infty}\left([\varepsilon, 1-\varepsilon]^{2}\right)$, where

$$
\left\{\mathbb{L}\left(\omega ; \tau_{1}, \tau_{2}\right)\right\}_{j_{1}, j_{2}}:=\frac{1}{\sqrt{\mathfrak{f}_{1,1} \mathfrak{f}_{2,2}}}\left(\mathbb{H}_{1,2}-\frac{1}{2} \frac{\mathfrak{f}_{1,2}}{\mathfrak{f}_{1,1}} \mathbb{H}_{1,1}-\frac{1}{2} \frac{\mathfrak{f}_{1,2}}{\mathfrak{f}_{2,2}} \mathbb{H}_{2,2}\right),
$$




$$
\left\{\boldsymbol{B}_{n}^{(k)}\left(\omega ; \tau_{1}, \tau_{2}\right)\right\}_{j_{1}, j_{2}}:=\frac{1}{\sqrt{\mathfrak{f}_{1,1} \mathfrak{f}_{2,2}}}\left(\boldsymbol{B}_{1,2}-\frac{1}{2} \frac{\mathfrak{f}_{1,2}}{\mathfrak{f}_{1,1}} \boldsymbol{B}_{1,1}-\frac{1}{2} \frac{\mathfrak{f}_{1,2}}{\mathfrak{f}_{2,2}} \boldsymbol{B}_{2,2}\right)
$$

and we have written $\mathfrak{f}_{a, b}$ for the quantile cross-spectral density $\mathfrak{f}^{j_{a}, j_{b}}\left(\omega ; \tau_{a}, \tau_{b}\right), \mathbb{H}_{a, b}$ for the limit distribution $\mathbb{H}^{j_{a}, j_{b}}\left(\omega ; \tau_{a}, \tau_{b}\right)$, and $\boldsymbol{B}_{a, b}$ for the bias $\left\{\boldsymbol{B}_{n}^{(k)}\left(\omega ; \tau_{a}, \tau_{b}\right)\right\}_{j_{a}, j_{b}}$ defined in Theorem $3.3(a, b=1,2)$.

Proof. Deferred to the Appendix (Section 7.4).

Comparing Theorem 3.4 with what is known for the traditional coherency (see, for example, Theorem 7.6.2 in Brillinger (1975)) we observe that the distribution of $\hat{\mathfrak{R}}_{n, R}\left(\omega ; \tau_{1}, \tau_{2}\right)$ is asymptotically equivalent to that of the traditional estimator [for a definition see (7.6.14) in Brillinger (1975)] computed from the unobserved time series (18).

The convergence to a Gaussian process in 20 can be employed to obtain asymptotically valid pointwise confidence bands. To this end, the covariance kernel of $\mathbb{L}$ can easily be determined from (21) and (17), yielding an expression similar to (7.6.16) in Brillinger (1975). A more detailed account on how to conduct inference is given in Section 7.1.2. Note that the bound to the order of the bias given in (7.6.15) in Brillinger (1975) applies to the expansion given in 22 .

If $W$ is a kernel of order $p \geq 1$ we have that the bias is of order $b_{n}^{p}$. Thus, if we choose the mean square error minimizing bandwidth $b_{n} \asymp n^{-1 /(2 p+1)}$ the bias will be of order $n^{-p /(2 p+1)}$. With this particular choice of $b_{n}$ we obtain $o\left(\left(n b_{n}\right)^{-1 / 4}\right)=o\left(n^{-1 /(4 p+2)}\right)$ and see that (19) holds.

Regarding the restriction $\varepsilon>0$, note that the convergence (20) can not hold if $\left(\tau_{1}, \tau_{2}\right)$ is on the border of the unit square, as the quantile coherency $\mathfrak{R}\left(\omega ; \tau_{1}, \tau_{2}\right)$ is not defined if $\tau_{j} \in\{0,1\}$, as this implies that $\operatorname{Var}\left(I\left\{F_{j}\left(X_{t, j}\right) \leq \tau_{j}\right\}\right)=0$.

\section{An example of a process generating quantile de- pendence across frequencies: $\operatorname{QVAR}(p)$}

For a better understanding of the dependence structures that we study in this paper, it is illustrative to introduce a process capable of generating them. We focus on generating dependence at different points of the joint distribution, which will vary across frequencies, but stay hidden from classical measures. In other words, we illustrate the intuition of spuriously independent variables, a situation when two variables seem to be independent when traditional cross-spectral analysis is used, while they are indeed clearly dependent at different parts of their joint distribution.

We base our example on a multivariate generalization of the popular quantile autoregression process (QAR) introduced by Koenker and Xiao (2006). Inspired by vector autoregression processes (VAR), we link multiple QAR processes through their lag structure and refer to the resulting process as a quantile vector autoregression process (QVAR). This provides a natural way of generating rich dependence structure between two random variables in points of their joint distribution and over different frequencies. The autocovariance function of a stationary QVAR(p) process is that of a fixed parameter VAR(p) process. This follows from the argument by Knight (2006), who concludes 
that the exclusive use of autocorrelations may thus "fail to identify structure in the data that is potentially very informative". We will show how quantile spectral analysis reveals what otherwise may remain invisible.

Let $\boldsymbol{X}_{t}=\left(X_{t, 1}, \ldots, X_{t, d}\right)^{\prime}, t \in \mathbb{Z}$, be a sequence of random vectors that fulfills

$$
\boldsymbol{X}_{t}=\sum_{j=1}^{p} \boldsymbol{\Theta}^{(j)}\left(\boldsymbol{U}_{t}\right) \boldsymbol{X}_{t-j}+\boldsymbol{\theta}^{(0)}\left(\boldsymbol{U}_{t}\right)
$$

where $\boldsymbol{\Theta}^{(1)}, \ldots, \boldsymbol{\Theta}^{(p)}$ are $d \times d$ matrices of functions, $\boldsymbol{\theta}^{(0)}$ is a $d \times 1$ column vector of functions, and $\boldsymbol{U}_{t}=\left(U_{t, 1}, \ldots, U_{t, d}\right)^{\prime}, t \in \mathbb{Z}$, is a sequence of independent vectors, with components $U_{t, k}$ that are $\mathcal{U}[0,1]$-distributed. We will assume that the elements of the th $\operatorname{row} \boldsymbol{\theta}_{\ell}^{(j)}\left(u_{\ell}\right)=\left(\theta_{\ell, 1}^{(j)}\left(u_{\ell}\right), \ldots, \theta_{\ell, d}^{(j)}\left(u_{\ell}\right)\right)$ of $\boldsymbol{\Theta}^{(j)}\left(u_{1}, \ldots, u_{d}\right)=\left(\boldsymbol{\theta}_{1}^{(j)}\left(u_{1}\right)^{\prime}, \ldots, \boldsymbol{\theta}_{d}^{(j)}\left(u_{d}\right)^{\prime}\right)^{\prime}$ and that the $\ell$ th element $\theta_{\ell}^{(0)}\left(u_{\ell}\right)$ of $\boldsymbol{\theta}^{(0)}=\left(\theta_{1}^{(0)}\left(u_{1}\right), \ldots, \theta_{d}^{(0)}\left(u_{d}\right)\right)^{\prime}$ only depend on the $\ell$ th variable, respectively. Note that in this design the $\ell$ th component of $\boldsymbol{U}_{t}$ determines the coefficients for the autoregression equation of the $\ell$ th component of $\boldsymbol{X}_{t}$. We refer to the process as a quantile vector autoregression process of order $p$, hence $\operatorname{QVAR}(p)$. In the bivariate case $(d=2)$ of order $p=1$, i.e. $\operatorname{QVAR}(1), 23$ takes the following form

$$
\left(\begin{array}{l}
X_{t, 1} \\
X_{t, 2}
\end{array}\right)=\left(\begin{array}{ll}
\theta_{11}^{(1)}\left(U_{t, 1}\right) & \theta_{12}^{(1)}\left(U_{t, 1}\right) \\
\theta_{21}^{(1)}\left(U_{t, 2}\right) & \theta_{22}^{(1)}\left(U_{t, 2}\right)
\end{array}\right)\left(\begin{array}{l}
X_{t-1,1} \\
X_{t-1,2}
\end{array}\right)+\left(\begin{array}{c}
\theta_{1}^{(0)}\left(U_{t, 1}\right) \\
\theta_{2}^{(0)}\left(U_{t, 2}\right)
\end{array}\right) .
$$

For the examples we assume that the components $U_{t, 1}$ and $U_{t, 2}$ are independent and set the components of $\boldsymbol{\theta}^{(0)}$ to $\theta_{1}^{(0)}(u)=\theta_{2}^{(0)}(u)=\Phi^{-1}(u), u \in[0,1]$, where $\Phi^{-1}(u)$ denotes the $u$-quantile of the standard normal distribution. Further, we set the diagonal elements of of $\boldsymbol{\Theta}^{(1)}$ to zero (i.e., $\left.\theta_{11}^{(1)}(u)=\theta_{22}^{(1)}(u)=0, u \in[0,1]\right)$ and the off-diagonal elements to $\theta_{12}^{(1)}(u)=\theta_{21}^{(1)}(u)=1.2(u-0.5), u \in[0,1]$. We thus create cross-dependence by linking the two processes with each other through the other ones lagged contributions. Note that this particular choice of parameter functions leads to the existence of a unique, strictly stationary solution (Bougerol and Picard, 1992). $\left(X_{t, 1}\right)_{t \in \mathbb{Z}}$ and $\left(X_{t, 2}\right)_{t \in \mathbb{Z}}$ are uncorrelated.

In Figure 3 the dynamics of the described QVAR(1) process are depicted. In terms of traditional coherency there appears to be no dependence across all frequencies. In terms of quantile coherency, on the other hand, rich dynamics are revealed in the different parts of the joint distribution. While, in the center of the distribution (at the $0.5 \mid 0.5$ level) the dependence is zero across frequencies, we see that the dependence increases if at least one of the quantile levels $\left(\tau_{1}, \tau_{2}\right)$ is chosen closer to 0 or 1 . More precisely, we see that the quantile coherency of this QVAR process resembles the shape of an VAR(1) process with coefficient matrix $\boldsymbol{\Theta}^{(1)}\left(\tau_{1}, \tau_{2}\right)$. The two processes are, for example when $\tau_{1}=0.05$ and $\tau_{2}=0.95$, clearly positively connected at lower frequencies with exactly the opposite value of quantile coherency at high frequencies, where the processes are in opposition. This also resembles the dynamics of the simple motivating examples from the introductory section of this paper, and highlights the importance of the quantile cross-spectral analysis as the dependence structure stays hidden if only the traditional measures are used.

In a second and third example, we consider a similar structure of parameters at the second and third lag. For the $\operatorname{QVAR}(2)$ process we let $\theta_{11}^{(j)}(u)=\theta_{22}^{(j)}(u)=0$, for $j=1,2$, 

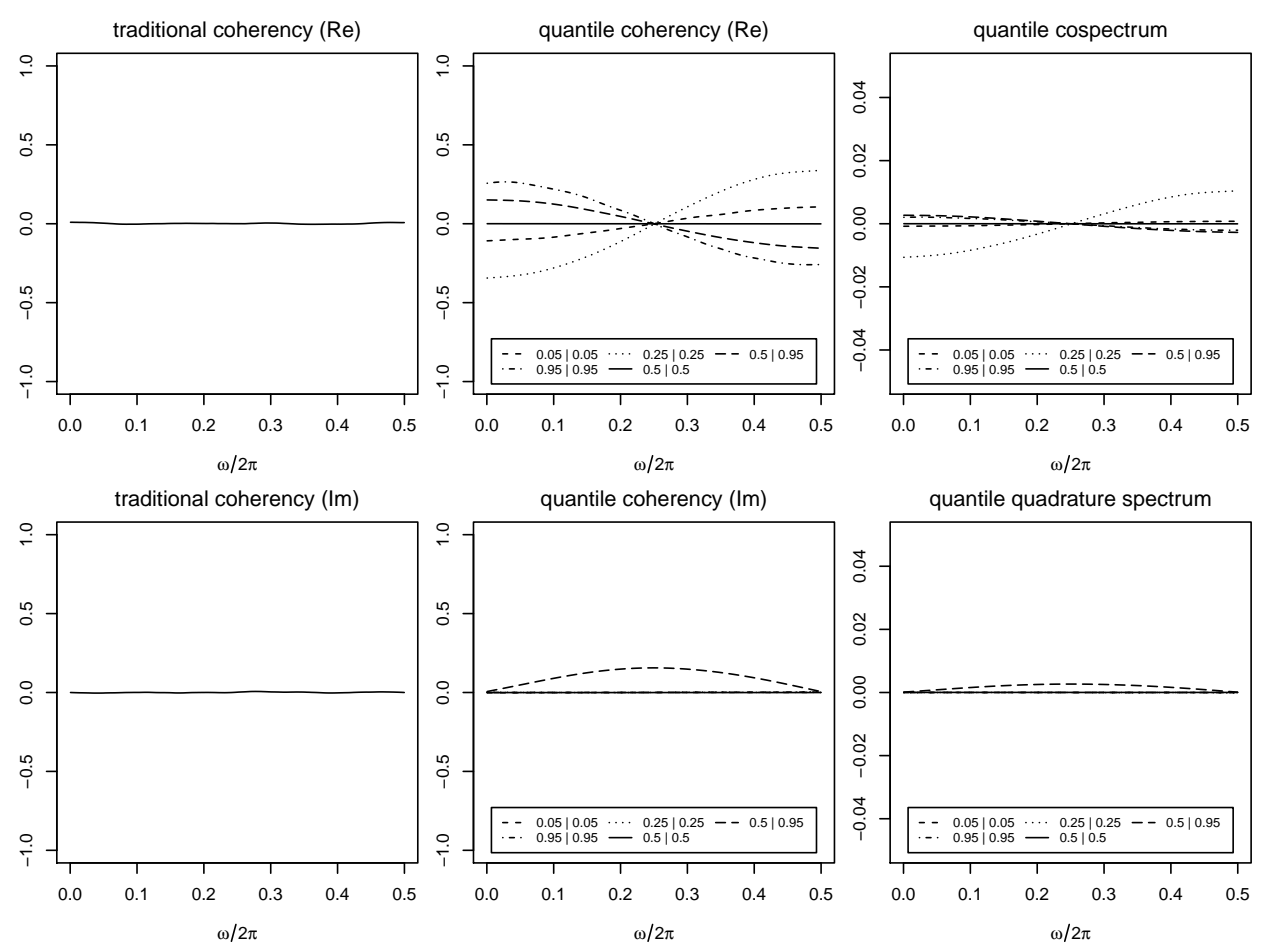

Figure 3: Example of dependence structures generated by $\operatorname{QVAR}(1)$ described by (23) with $\theta_{1}^{(0)}(u)=\theta_{2}^{(0)}(u)=\Phi^{-1}(u), \theta_{11}^{(1)}(u)=\theta_{22}^{(1)}(u)=0$, and $\theta_{12}^{(1)}(u)=\theta_{21}^{(1)}(u)=1.2(u-0.5), u \in[0,1]$. Left column: traditional coherency. Middle column: quantile-coherency for some combinations of $\tau_{1}, \tau_{2} \in\{0.05,0.25,0.5,0.95\}$. Right column: quantile cospectrum and quantile quadrature spectrum. Figure shows real and imaginary parts of the complex valued quantities.

$\theta_{12}^{(1)}(u)=\theta_{21}^{(1)}(u)=0$ and $\theta_{12}^{(2)}(u)=\theta_{21}^{(2)}(u)=1.2(u-0.5)$. In other words, here, the processes are connected through the second lag of the other one and, again, not directly through their own lagged contributions. In the QVAR $(3)$ process, all coefficients are again set to zero, except for $\theta_{12}^{(3)}(u)=\theta_{21}^{(3)}(u)=1.2(u-0.5)$, such that the processes are connected only through the third lag of the other component and not through their own contributions.

In Figures 4 and 5 the dynamics of the described QVAR $(2)$ and QVAR(3) processes are shown. Connecting the quantiles of the two processes through the second and third lag gives us richer dependence structures across frequencies. They, again, resemble the shape of the traditional coherencies of $\operatorname{VAR}(2)$ and $\operatorname{VAR}(3)$ processes. When traditional coherency is used for the $\operatorname{QVAR}(2)$ and $\operatorname{QVAR}(3)$ processes, the dependence structure stays completely hidden.

These examples of the general $\operatorname{QVAR}(p)$ specified in 23 served to show how rich dependence structures can be created across points of the joint distribution and different frequencies. It is obvious, how more complicated structures for the coefficient functions would lead to even richer dynamics than in the examples shown. 

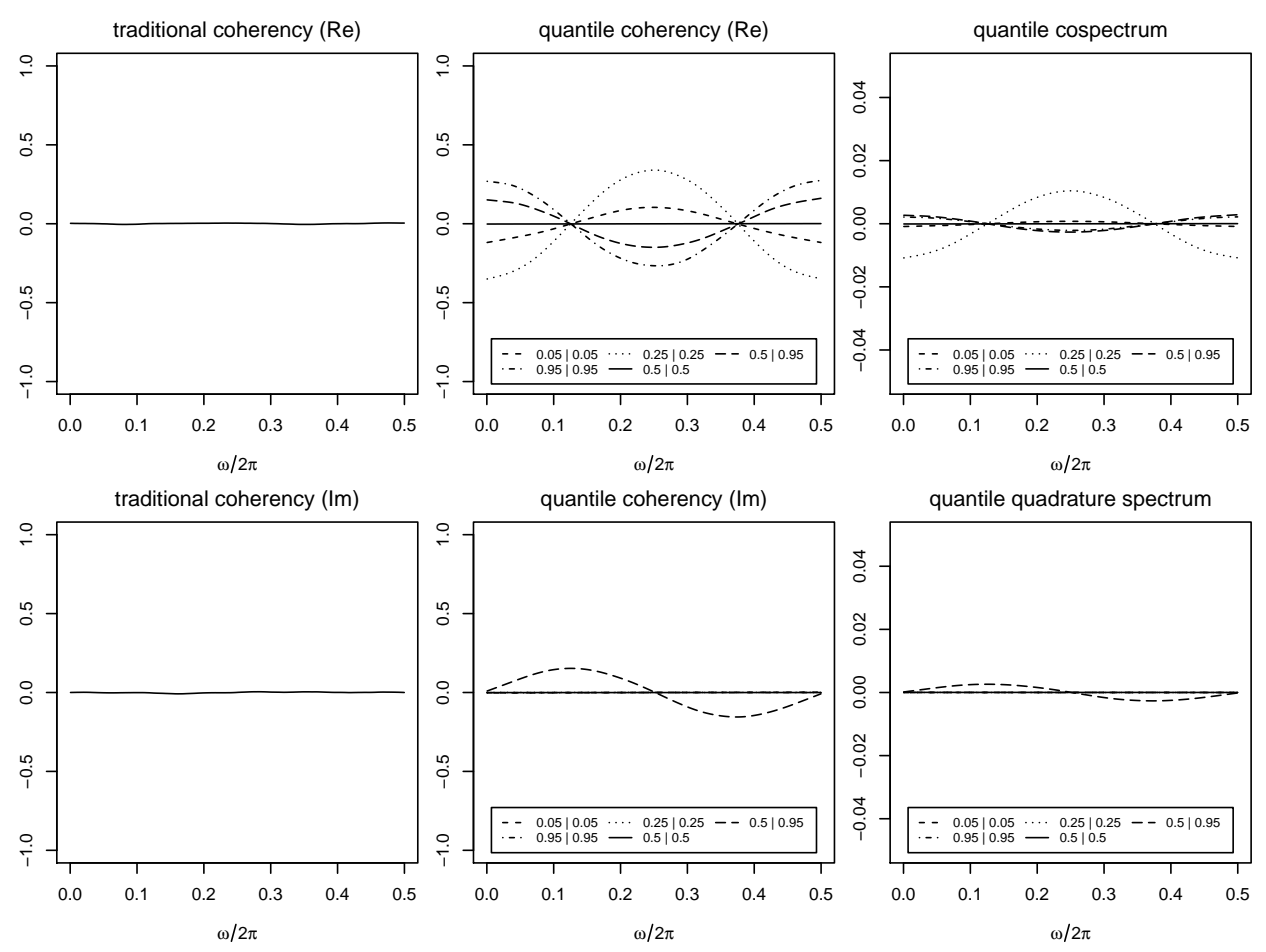

Figure 4: Example of dependence structures generated by $\operatorname{QVAR}(2)$ described by (23) with $\theta_{1}^{(0)}(u)=\theta_{2}^{(0)}(u)=\Phi^{-1}(u), \theta_{11}^{(1)}(u)=\theta_{22}^{(1)}(u)=\theta_{11}^{(2)}(u)=\theta_{22}^{(2)}(u)=\theta_{12}^{(1)}(u)=\theta_{21}^{(1)}(u)=0$ and $\theta_{12}^{(2)}(u)=\theta_{21}^{(2)}(u)=1.2(u-0.5), u \in[0,1]$. Left column: traditional coherency. Middle column: quantile-coherency for some combinations of $\tau_{1}, \tau_{2} \in\{0.05,0.25,0.5,0.95\}$. Right column: quantile cospectrum and quantile quadrature spectrum. Figure shows real and imaginary parts of the complex valued quantities.

\section{Quantile cross-spectral analysis of stock market re- turns: A route to more accurate risk measures?}

Stock market returns belong to the most prominent datasets in economics and finance. Although many important stylized facts about their behavior have been established in the past decades, it remains a very active area of research. Despite the efforts, an important direction, which has not been fully addressed is stylized facts about the joint distribution of returns. Especially during the last turbulent decade, understanding the behavior of joint quantiles in return distributions became particularly important, as it is essential for understanding systemic risk; "the risk that the intermediation capacity of the entire system can be impaired" (Adrian and Brunnermeier, 2011). Several authors focus on explaining tails of the bivariate market distributions in different ways. Adrian and Brunnermeier (2011) proposed to classify institutions according to the sensitivity of their quantiles to shocks to the market. Han et al. (2014) proposed cross-quantilograms and used them to measure co-dependence in the tails of equity returns of an individual institution and of the whole system. Most closely related to the notion of how we view the dependence structures is the recently proposed multivariate regression quantile model 

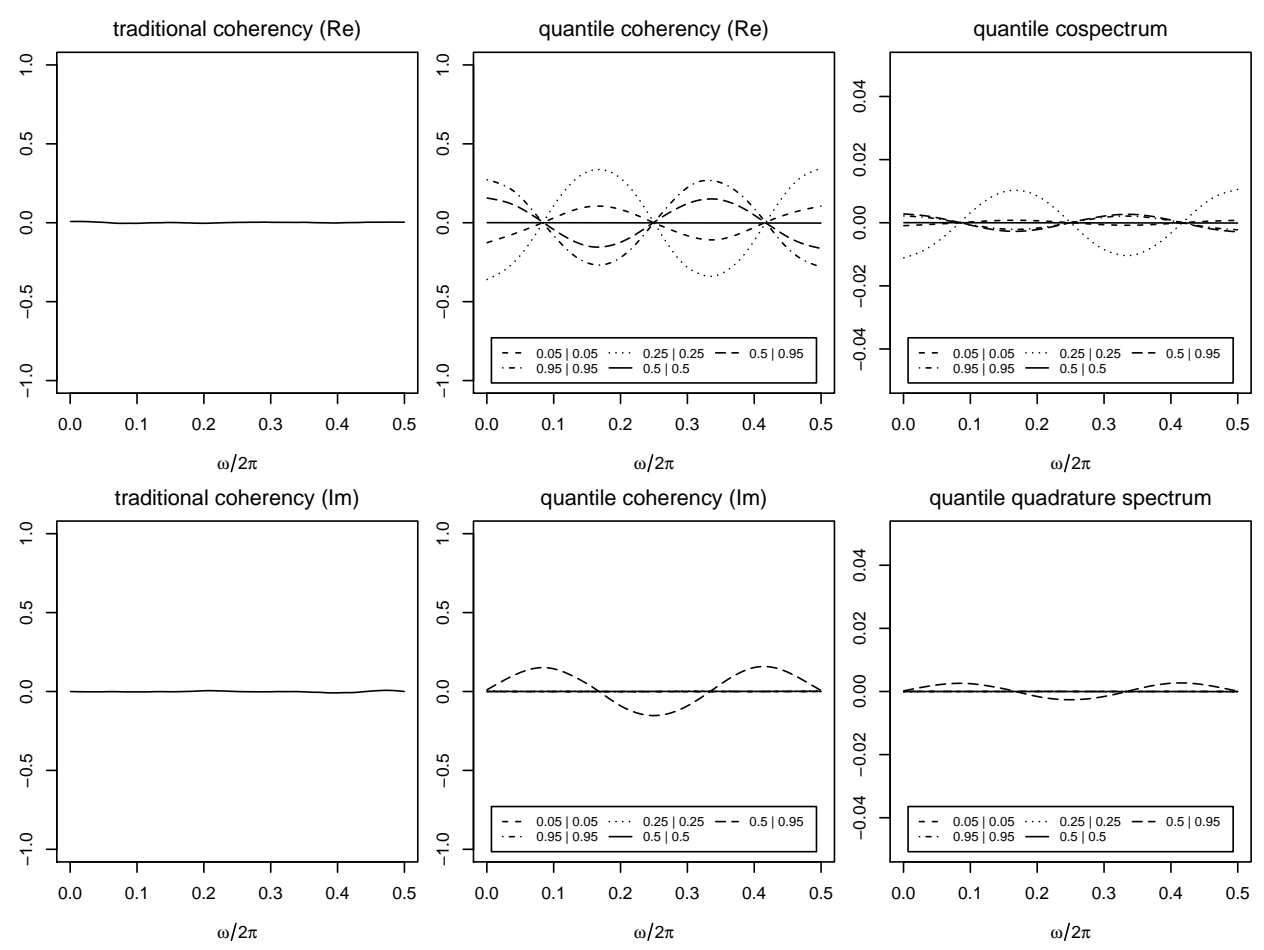

Figure 5: Example of dependence structures generated by $\operatorname{QVAR}(3)$ described by (23) with $\theta_{1}^{(0)}(u)=\theta_{2}^{(0)}(u)=\Phi^{-1}(u), \theta_{11}^{(j)}(u)=\theta_{22}^{(j)}(u)=\theta_{12}^{(k)}(u)=\theta_{21}^{(k)}(u)=0$, for $j=1,2,3$ and $k=1,2$, and $\theta_{12}^{(3)}(u)=\theta_{21}^{(3)}(u)=1.2(u-0.5), u \in[0,1]$. Left column: traditional coherency. Middle column: quantile-coherency for some combinations of $\tau_{1}, \tau_{2} \in\{0.05,0.25,0.5,0.95\}$. Right column: quantile cospectrum and quantile quadrature spectrum. Figure shows real and imaginary parts of the complex valued quantities.

of White et al. (2015), which studies the degree of tail interdependence among different random variables directly.

Quantile cross-spectral analysis, as designed in this paper, allows to estimate the fundamental dependence quantities in the tails (but also in any other part) of the joint distribution and across frequencies. An application to stock market returns may therefore provide deeper insight about dependence in stock markets, and lead to a more powerful analysis securing us against financial collapses.

One of the most prominent features of stock market returns is time variation in its volatility. Time-varying volatility processes can cross almost every quantile of their distribution (Hagemann, 2013), and create peaks in quantile spectral densities as shown by Li (2014). These notions have recently been documented by Engle and Manganelli (2004) and Żikeš and Baruník (2014) who propose models for the conditional quantiles of the return distribution based on the past volatility. In the multivariate setting, strong common factors in volatility are found by Barigozzi et al. (2014) who conclude that common volatility is an important risk factor. Hence, common volatility should be viewed as a possible source of dependence. Because we aim to find the common structures in the joint distribution of returns, we study returns standardized by its volatility that we estimate by a $\operatorname{GARCH}(1,1)$ model (Bollerslev, 1986). This first step is commonly taken in the 
literature of modeling the joint market distribution using copulas (Granger et al., 2006 , Patton, 2012). In these approaches the volatility in the marginal distributions is modeled first, and the common factors are then considered in the second step. Consequently, this will allow us to discover other possible common factors in the joint distribution of market returns across frequencies, that result in spurious dependence, but which will not be overshadowed by the strong volatility process.
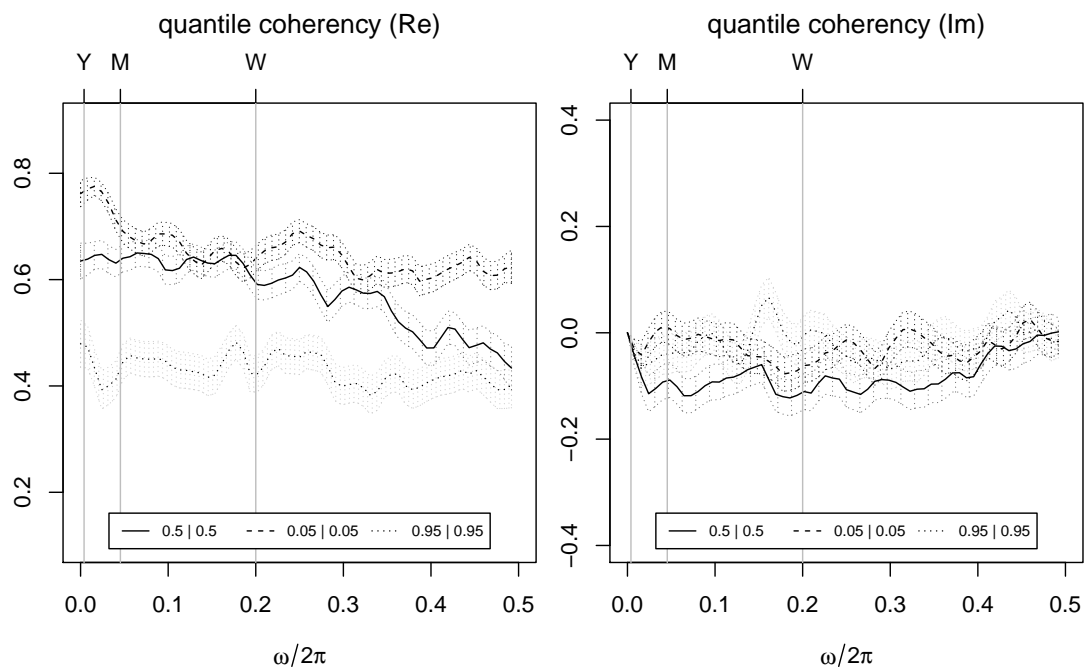

Figure 6: Real (left) and imaginary (right) parts of the quantile coherency estimates for the portfolio formed from consumer non-durables and excess market returns for 0.05, 0.5, and 0.95 quantiles together with $95 \%$ confidence intervals. W, M, and Y denotes weekly, monthly, and yearly periods. Data span years $1926-2015$.

We choose to study the joint distribution of portfolio returns and excess returns on the broad market, hence looking at one of the most commonly studied factor structures in the literature as dictated by asset pricing theories (Sharpe, 1964; Lintner, 1965). As an excess return on the market, we use value-weighted returns of all firms listed on the NYSE, AMEX, or NASDAQ from the Center for Research in Security Price (CRSP) database. For the benchmark portfolio, we use an industry portfolio formed from consumer nondurables ${ }^{3}$ The daily data we have used runs from July 1, 1926 through to June 30, 2015.

Although very attractive due to powerful and intuitive predictions about risk measurement, the capital asset pricing theory (CAPM) is largely invalidated empirically by researchers (Fama and French, 2004). In the model it is assumed that the variance of the returns is an adequate risk measure, as implied by the assumption of the returns under study being normally distributed. Intuitively, departures from normality are better capable of capturing the investor's preferences. Risk can be viewed as a probability of loss,

\footnotetext{
${ }^{3}$ Note to choice of the data: we use the publicly available data available and maintained by Fama and French at http://mba.tuck.dartmouth.edu/pages/faculty/ken.french/data_library.html. This data set is popular among researchers, and while many types of portfolios can be chosen, we chose consumer non-durables randomly for this application. Although very interesting and attractive, it is far beyond the scope of this work to present and discuss results for wider portfolios formed on distinct criteria.
} 
hence is asymmetric, implying that we should move our focus from variability of returns to their quantiles directly.

In Figure 6, quantile coherency estimates for the $0.05|0.05,0.5| 0.5$, and $0.95 \mid 0.95$ combinations of quantile levels of the joint distribution are shown for the industry portfolio and excess market returns over frequencies. For clarity, we plot the $x$-axis in daily cycles and also indicate the frequencies that correspond to yearly, monthly, and weekly periods. While we use daily data the highest possible frequency of 0.5 indicates 0.5 cycles per day (i. e., a 2-day period). While precise frequencies do not have an economic meaning, one needs to understand the interpretation with respect to the time domain. For example, a sampling frequency of 0.2 corresponds to 0.2 cycles per day translating to a 5 days period (equivalent to one week), but the frequency of 0.3 translates to a hardly interpretable $3 . \overline{3}$ period. Hence, the upper label of the $x$-axis is of particular interest to an economist, as one can study how weekly, monthly, or yearly cycles are connected across quantiles of the joint distribution.

The real parts of the quantile coherency estimates reveal frequency dynamics in quantiles of the joint distribution of the returns under study. Generally, lower quantiles are more strongly dependent than upper quantiles, which is a well documented stylized fact about stock market returns. It points us to the fact that returns are more dependent during business cycle downturns, than upturns (Erb et al., 1994; Longin and Solnik, 2001; Ang and Chen, 2002; Patton, 2012). More importantly, lower quantiles as well as the median are strongly related in periods longer than one week in average in comparison to shorter than weekly periods, and are even more connected at longer than monthly cycles. This suggests that infrequent clusters of large negative portfolio returns are better explained by excess market returns than small daily fluctuations. The same result holds also for the median, while returns in upper quantiles of the joint distribution seem to be connected similarly across all frequencies.

While asymmetry is commonly found by researchers, we document frequency dependent asymmetry in the joint distribution of stock market returns. In case this behavior would be common across larger classes of assets, our results may have large implications for one of the cornerstones of asset pricing theory assuming normal distribution of returns. It leads us to the call for more general models, and more importantly to the need of restating the asset pricing theory in a way that allows to distinguish between short run and long run behavior of investors.

Our results are also crucial for systemic risk measurement, as an investor wishing to optimize a portfolio should focus on stocks which will not be connected at lower quantiles, in a situation of distress, but will be connected at upper quantiles, in a situation of market upturns in a given investment period. We document behavior which is not favorable to such an investor using traditional pricing theories, as we show that broad stock market returns contain a common factor more frequently during downturns than during upturns. This suggests that the portfolio at hand might be much riskier than it were implied by common measures. Further, our results suggest that this effect becomes even worse for long-run investors.

An important feature of our quantile cross-spectral measures is that they enable us to measure dependence also between $\tau_{1} \neq \tau_{2}$ quantiles of the joint distribution. In Figure 7 we document that the dependence between the $0.05 \mid 0.95$ quantiles of the return 

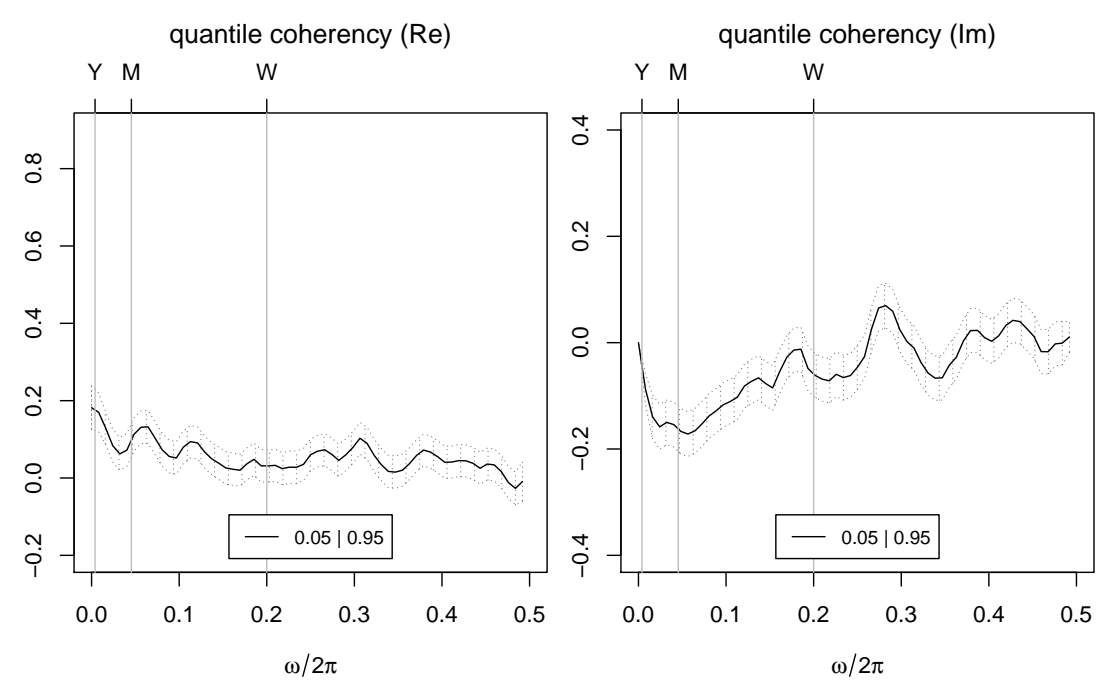

Figure 7: Real (left) and imaginary (right) parts of the quantile coherency estimates for the 0.05 quantile of portfolio formed from consumer non-durables and 0.95 quantile of excess market returns together with $95 \%$ confidence intervals. W, M, and Y denotes weekly, monthly, and yearly periods, respectively. The data spans the years 1926-2015.

distribution is not very strong. Generally speaking, no dependence can be seen between large negative returns of the stock market, and large positive returns of the portfolio under study. This analysis may be much more interesting in case of dependence between individual assets, where negative news may have strong opposite impact on the assets under study.

Here, we remind the reader about the interpretation of the quantities which we have estimated. In Section 2.3 we provided a link between quantile coherency and traditional measures of dependence under the assumption of normally distributed data. The quantile-based measures are designed to capture general dependence types without restrictive assumptions on the underlying distribution of the process. Hence, we do intentionally not relate it to traditional correlation which, ideally, should only be interpreted when the process is known to be Gaussian. The financial returns under study in this section are known to depart from normality. Therefore, quantile coherency is not directly comparable to traditional correlation measures. What we can see is generally very strong dependence between the portfolio returns and excess market returns at all quantiles confirming the fact that excess returns are a strong common factor for the studied portfolio returns. The details that the quantile-based analysis in this section revealed would have remained hidden in an analysis based on the traditional coherency.

\section{Conclusion}

In this paper we introduced quantile cross-spectral analysis of economic time series providing an entirely model-free, nonparametric theory for the estimation of general crossdependence structures emerging from quantiles of the joint distribution in the frequency 
domain. We argue that complex dynamics in time series often arise naturally in many macroeconomic and financial time series, as infrequent periods of large negative values (lower quantiles of the joint distribution) may be more dependent than infrequent periods of large positive values (upper quantiles of the joint distribution). Moreover, the dependence may differ in the long-, medium, or short-run. Quantile cross-spectral analysis hence fundamentally changes the way how we view the dependence between economic time series, and may be viewed as a precursor to the subsequent developments in economic research underlying many new modeling strategies.

Bridging two literatures which focus on the dependence between variables in quantiles of their joint distribution and across frequencies separately, the proposed methods may be viewed as an important step in robustifying the traditional cross-spectral analysis as well. Quantile-based spectral quantities are very attractive as they do not require the existence of moments, an important relaxation to the classical assumptions, where moments up to the order of the cumulants involved are typically assumed to exist. The proposed quantities are robust to many common violations of traditional assumptions found in data, including outliers, heavy tails, and changes in higher moments of the distribution. By considering quantiles instead of moments the proposed methods are able to reveal the dependence even in uncorrelated data. As an essential ingredient for a successful applications we have provided a rigorous analysis of the asymptotic properties of the introduced estimators and showed that for a general class of nonlinear processes, properly centered and smoothed versions of the quantile-based estimators converge in distribution to centered Gaussian processes.

In an empirical application, we have shown that asset pricing theories may not suit the data well, as commonly documented by researchers, because rich dependence structures exists varying across quantiles and frequencies in the joint distribution of returns. We document strong dependence of the bivariate returns series in periods of large negative returns, while positive returns display less dependence over all frequencies. This result is not favorable for an investor, as exactly the opposite would be desired: choosing to invest to a stocks with independent negative returns, but dependent positive returns. Our tool reveals that systematic risk originates more strongly from lower quantiles of the joint distribution in the long-, and medium-run investment horizons in comparison to the upper quantiles.

We believe that our work opens many exciting routes for future theoretical as well as empirical research. From the perspective of applications, exploratory analysis based on the quantile cross-spectral estimators can reveal new implications for improvement or even restating of many economic problems. Dependence in many economic time series

is of a non-Gaussian nature, calling for an escape from covariance-based methods and allowing for a detailed analysis of the dependence in the quantiles of the joint distribution.

\section{References}

Adrian, T. and M. K. Brunnermeier (2011). CoVaR. http://www.nber.org/papers/ w17454. Technical report, National Bureau of Economic Research. 
Ang, A. and J. Chen (2002). Asymmetric correlations of equity portfolios. Journal of Financial Economics 63(3), 443-494.

Bae, K.-H., G. A. Karolyi, and R. M. Stulz (2003). A new approach to measuring financial contagion. Review of Financial studies 16(3), 717-763.

Barigozzi, M., C. Brownlees, G. M. Gallo, and D. Veredas (2014). Disentangling systematic and idiosyncratic dynamics in panels of volatility measures. Journal of Econometrics 182(2), 364-384.

Beaudry, P. and G. Koop (1993). Do recessions permanently change output? Journal of Monetary economics 31(2), 149-163.

Birr, S., S. Volgushev, T. Kley, H. Dette, and M. Hallin (2015). Quantile spectral analysis for locally stationary time series (arxiv:1404.4605v2). ArXiv e-prints.

Bollerslev, T. (1986). Generalized autoregressive conditional heteroskedasticity. Journal of Econometrics $31(3), 307-327$.

Bougerol, P. and N. Picard (1992). Strict stationarity of generalized autoregressive processes. The Annals of Probability 20(4), 1714-1730.

Boussama, F. (1998). Ergodicité, mélange et estimation dans le modelés GARCH. Ph. D. thesis, Université 7 Paris.

Brillinger, D. R. (1975). Time Series: Data Analysis and Theory. New York: Holt, Rinehart and Winston, Inc.

Brockwell, P. J. and R. A. Davis (1987). Time Series: Theory and Methods. Springer Series in Statistics. New York: Springer.

Carrasco, M. and X. Chen (2002). Mixing and moment properties of various GARCH and stochastic volatility models. Econometric Theory 18(1), 17-39.

Croux, C., M. Forni, and L. Reichlin (2001). A measure of comovement for economic variables: Theory and empirics. Review of Economics and Statistics 83(2), 232-241.

Davis, R. A., T. Mikosch, et al. (2009). The extremogram: A correlogram for extreme events. Bernoulli 15(4), 977-1009.

Dette, H., M. Hallin, T. Kley, and S. Volgushev (2015). Of copulas, quantiles, ranks and spectra: An $L_{1}$-approach to spectral analysis. Bernoulli 21(2), 781-831.

Enders, W. and C. W. J. Granger (1998). Unit-root tests and asymmetric adjustment with an example using the term structure of interest rates. Journal of Business Eamp; Economic Statistics 16(3), 304-311.

Engle, R. F. and S. Manganelli (2004). CAViaR: Conditional autoregressive value at risk by regression quantiles. Journal of Business 85 Economic Statistics 22(4), 367-381. 
Erb, C. B., C. R. Harvey, and T. E. Viskanta (1994). Forecasting international equity correlations. Financial Analysts Journal 50(6), 32-45.

Fama, E. F. and K. R. French (2004). The capital asset pricing model: Theory and evidence. Journal of Economic Perspectives 18, 25-46.

Fan, Y. and A. J. Patton (2014). Copulas in econometrics. Annual Review of Economics 6(1), 179-200.

Francq, C. and J.-M. Zakoïan (2006). Mixing properties of a general class of GARCH $(1,1)$ models without moment assumptions on the observed process. Econometric Theory 22(05), 815-834.

Granger, C. W. (1966). The typical spectral shape of an economic variable. Econometrica, $150-161$.

Granger, C. W. (1969). Investigating causal relations by econometric models and crossspectral methods. Econometrica, 424-438.

Granger, C. W. (2010). Some thoughts on the development of cointegration. Journal of Econometrics 158(1), 3-6.

Granger, C. W. and P. Newbold (1974). Spurious regressions in econometrics. Journal of Econometrics 2(2), 111-120.

Granger, C. W., T. Teräsvirta, and A. J. Patton (2006). Common factors in conditional distributions for bivariate time series. Journal of Econometrics 132(1), 43-57.

Hagemann, A. (2013). Robust spectral analysis (arxiv:1111.1965v2). ArXiv e-prints.

Han, H., O. Linton, T. Oka, and Y.-J. Whang (2014). The cross-quantilogram: measuring quantile dependence and testing directional predictability between time series (arxiv:1402.1937v1). ArXiv e-prints.

Hill, J. B. and A. McCloskey (2014). Heavy tail robust frequency domain estimation. http://www.econ.brown.edu/fac/adam_mccloskey/Research_ files/FDTTQML.pdf.

Hong, Y. (1999). Hypothesis testing in time series via the empirical characteristic function: a generalized spectral density approach. Journal of the American Statistical Association 94(448), 1201-1220.

Hong, Y. (2000). Generalized spectral tests for serial dependence. Journal of the Royal Statistical Society Series B 62(3), 557-574.

Katkovnik, V. (1998). Robust M-periodogram. IEEE Transactions on Signal Processing 46(11), 3104-3109.

Kleiner, R., R. D. Martin, and D. J. Thomson (1979). Robust estimation of power spectra. Journal of the Royal Statistical Society Series B 41(3), 313-351. 
Kley, T. (2014). Quantile-based spectral analysis in an object-oriented framework and a reference implementation in R: The quantspec package (arxiv:1408.6755). ArXiv e-prints.

Kley, T. (2015). quantspec: Quantile-based spectral analysis functions. R package version $1.2-0$.

Kley, T., S. Volgushev, H. Dette, and M. Hallin (2015+). Quantile spectral processes: Asymptotic analysis and inference. Bernoulli forthcoming.

Klüppelberg, C. and T. Mikosch (1994). Some limit theory for the self-normalised periodogram of stable processes. Scandinavian Journal of Statistics 21, 485-491.

Knight, K. (2006). Comment on "Quantile autoregression". Journal of the American Statistical Association 101(475), 994-996.

Koenker, R. (2005). Quantile Regression. Econometric Society Monographs. Cambridge University Press.

Koenker, R. and Z. Xiao (2006). Quantile autoregression. Journal of the American Statistical Association 101(475), 980-990.

Lee, J. and S. S. Rao (2012). The quantile spectral density and comparison based tests for nonlinear time series (arxiv:1112.2759v2). ArXiv e-prints.

Li, G., Y. Li, and C.-L. Tsai (2015). Quantile correlations and quantile autoregressive modeling. Journal of the American Statistical Association 110(509), 246-261.

Li, T.-H. (2008). Laplace periodogram for time series analysis. Journal of the American Statistical Association 103(482), 757-768.

Li, T.-H. (2012). Quantile periodograms. Journal of the American Statistical Association 107(498), 765-776.

Li, T.-H. (2014). Quantile periodogram and time-dependent variance. Journal of Time Series Analysis 35(4), 322-340.

Liebscher, E. (2005). Towards a unified approach for proving geometric ergodicity and mixing properties of nonlinear autoregressive processes. Journal of Time Series Analysis $26(5), 669-689$.

Lintner, J. (1965). The valuation of risk assets and the selection of risky investments in stock portfolios and capital budgets. The Review of Economics 85 Statistics, 13-37.

Linton, O. and Y.-J. Whang (2007). The quantilogram: With an application to evaluating directional predictability. Journal of Econometrics 141(1), 250-282.

Longin, F. and B. Solnik (2001). Extreme correlation of international equity markets. Journal of Finance, 649-676. 
Maronna, R., D. Martin, and V. Yohai (2006). Robust Statistics: Theory and Methods. Wiley Series in Probability and Statistics. Wiley.

Mikosch, T. and Y. Zhao (2014). A fourier analysis of extreme events. Bernoulli 20(2), 803-845.

Mikosch, T. and Y. Zhao (2015). The integrated periodogram of a dependent extremal event sequence. Stochastic Processes and their Applications 125(8), 3126-3169.

Neftci, S. N. (1984). Are economic time series asymmetric over the business cycle? The Journal of Political Economy, 307-328.

Ning, C. Q. and L. Chollete (2009). The dependence structure of macroeconomic variables in the us. http://www1.uis.no/ansatt/odegaard/uis_wps_econ_fin/uis_ wps_2009_31_chollete_ning.pdf. Technical report.

Patton, A. J. (2012). A review of copula models for economic time series. Journal of Multivariate Analysis 110, 4-18.

Schmitt, T. A., R. Schäfer, H. Dette, and T. Guhr (2015). Quantile correlations: Uncovering temporal dependencies in financial time series. Journal of Theoretical and Applied Finance forthcoming.

Shao, X. and J. Zhang (2014). Martingale difference correlation and its use in high-dimensional variable screening. Journal of the American Statistical Association 109 (507), 1302-1318.

Sharpe, W. F. (1964). Capital asset prices: A theory of market equilibrium under conditions of risk. The Journal of Finance 19(3), 425-442.

Székely, G. J., M. L. Rizzo, and N. K. Bakirov (2007, 12). Measuring and testing dependence by correlation of distances. Ann. Statist. 35(6), 2769-2794.

Taniguchi, M. and Y. Kakizawa (2000). Asymptotic theory of statistical inference for time series. Springer.

van der Vaart, A. and J. Wellner (1996). Weak Convergence and Empirical Processes: With Applications to Statistics. New York: Springer.

White, H., T.-H. Kim, and S. Manganelli (2015). VAR for VaR: Measuring tail dependence using multivariate regression quantiles. Journal of Econometrics 187(1), 169-188.

Xiao, Z. (2009). Quantile cointegrating regression. Journal of Econometrics 150(2), $248-260$.

Zhou, Z. (2012). Measuring nonlinear dependence in time-series, a distance correlation approach. Journal of Time Series Analysis 33(3), 438-457.

Žikeš, F. and J. Baruník (2014). Semi-parametric conditional quantile models for financial returns and realized volatility. Journal of Financial Econometrics, forthcoming. 


\section{Technical Appendix}

In the first section of the appendix we collect details on how to construct pointwise confidence bands. The proofs to the theorems in the paper are given in the remaining sections of the appendix.

\subsection{Interval Estimators}

Section 3 contained asymptotic results on the uncertainty of point estimation of the newly introduced quantile cross-spectral quantities. In this section we describe strategies to estimate the variances (of the real and imaginary parts) that appear in those limit results and describe how asymptotically valid pointwise confidence bands can be constructed.

In all three subsections the following comment is relevant. Assuming that we have determined the weights $W_{n}$ form a kernel $W$ that is of order $d$. We will choose a bandwidth $b_{n}=o\left(n^{-1 /(2 d+1)}\right)$. This choice implies that compared to the variance the bias (that in some form appears in both limit results) is asymptotically negligible: $\sqrt{n b_{n}} \boldsymbol{B}_{n}^{(k)}\left(\omega ; \tau_{1}, \tau_{2}\right)=o(1)$.

\subsubsection{Pointwise Confidence Bands for $\mathfrak{f}$}

Utilizing Theorem 3.3 we now construct pointwise asymptotic $(1-\alpha)$-level confidence bands for the real and imaginary parts of $\mathfrak{f}^{j_{1}, j_{2}}\left(\omega_{k n} ; \tau_{1}, \tau_{2}\right), \omega_{k n}:=2 \pi k / n$, as follows:

$$
C_{\mathrm{r}, n}^{(1)}\left(\omega_{k n} ; \tau_{1}, \tau_{2}\right):=\Re \tilde{G}_{n, R}^{j_{1}, j_{2}}\left(\omega_{k n} ; \tau_{1}, \tau_{2}\right) \pm \Re \sigma_{(1)}^{j_{1}, j_{2}}\left(\omega_{k n} ; \tau_{1}, \tau_{2}\right) \Phi^{-1}(1-\alpha / 2),
$$

for the real part, and

$$
C_{\mathrm{i}, n}^{(1)}\left(\omega_{k n} ; \tau_{1}, \tau_{2}\right):=\Im \tilde{G}_{n, R}^{j_{1}, j_{2}}\left(\omega_{k n} ; \tau_{1}, \tau_{2}\right) \pm \Im \sigma_{(1)}^{j_{1}, j_{2}}\left(\omega_{k n} ; \tau_{1}, \tau_{2}\right) \Phi^{-1}(1-\alpha / 2),
$$

for the imaginary part of the quantile cross-spectrum. Here,

$$
\tilde{G}_{n, R}^{j_{1}, j_{2}}\left(\omega_{k n} ; \tau_{1}, \tau_{2}\right):=\hat{G}_{n, R}^{j_{1}, j_{2}}\left(\omega_{k n} ; \tau_{1}, \tau_{2}\right) / W_{n}^{k}, \quad W_{n}^{k}:=\frac{2 \pi}{n} \sum_{s=1}^{n-1} W_{n}\left(\omega_{k n}-\omega_{s n}\right),
$$

and $\Phi$ denotes the cumulative distribution function of the standard normal distribution ${ }^{4}$

$$
\left(\Re \sigma^{j_{1}, j_{2}}\left(\omega_{k n} ; \tau_{1}, \tau_{2}\right)\right)^{2}:=0 \vee \begin{cases}\operatorname{Cov}\left(\mathbb{H}_{1,2}, \mathbb{H}_{1,2}\right) & \text { if } j_{1}=j_{2} \text { and } \tau_{1}=\tau_{2}, \\ \frac{1}{2}\left(\operatorname{Cov}\left(\mathbb{H}_{1,2}, \mathbb{H}_{1,2}\right)+\Re \operatorname{Cov}\left(\mathbb{H}_{1,2}, \mathbb{H}_{2,1}\right)\right) & \text { otherwise, }\end{cases}
$$

and

$$
\left(\Im \sigma^{j_{1}, j_{2}}\left(\omega_{k n} ; \tau_{1}, \tau_{2}\right)\right)^{2}:=0 \vee \begin{cases}0 & \text { if } j_{1}=j_{2} \text { and } \tau_{1}=\tau_{2}, \\ \frac{1}{2}\left(\operatorname{Cov}\left(\mathbb{H}_{1,2}, \mathbb{H}_{1,2}\right)-\Re \operatorname{Cov}\left(\mathbb{H}_{1,2}, \mathbb{H}_{2,1}\right)\right) & \text { otherwise, }\end{cases}
$$

\footnotetext{
${ }^{4}$ Note that for $k=0, \ldots, n-1$ we have $W_{n}^{k}:=2 \pi / n \sum_{0=s \neq k} W_{n}(2 \pi s / n)$. For $k \in \mathbb{Z}$ with $k<0$ or $k \geq n$ we can define it as the $n$ periodic extension.
} 
where $\operatorname{Cov}\left(\mathbb{H}_{a, b}, \mathbb{H}_{c, d}\right)$ denotes an estimator of $\operatorname{Cov}\left(\mathbb{H}^{j_{a}, j_{b}}\left(\omega_{k n} ; \tau_{a}, \tau_{b}\right), \mathbb{H}^{j_{c}, j_{d}}\left(\omega_{k n} ; \tau_{c}, \tau_{d}\right)\right)$. Here, motivated by Theorem 7.4.3 in Brillinger (1975), we use

$$
\begin{aligned}
& \left(\frac{2 \pi}{n \cdot W_{n}^{k}}\right) \times\left[\sum_{s=1}^{n-1} W_{n}(2 \pi(k-s) / n) W_{n}(2 \pi(k-s) / n) \tilde{G}_{n, R}^{j_{a}, j_{c}}\left(\tau_{a}, \tau_{c} ; 2 \pi s / n\right) \tilde{G}_{n, R}^{j_{b}, j_{d}}\left(\tau_{b}, \tau_{d} ;-2 \pi s / n\right)\right. \\
& \left.\quad+\sum_{s=1}^{n-1} W_{n}(2 \pi(k-s) / n) W_{n}(2 \pi(k+s) / n) \tilde{G}_{n, R}^{j_{a}, j_{d}}\left(\tau_{a}, \tau_{d} ; 2 \pi s / n\right) \tilde{G}_{n, R}^{j_{b}, j_{c}}\left(\tau_{b}, \tau_{c} ;-2 \pi s / n\right)\right]
\end{aligned}
$$

The definition of $\sigma_{(1)}^{j_{1}, j_{2}}\left(\omega_{k n} ; \tau_{1}, \tau_{2}\right)$ is motivated by the fact that $\Im \hat{G}_{n, R}^{j_{1}, j_{2}}\left(\omega_{k n} ; \tau_{1}, \tau_{2}\right)=0$, if $j_{1}=j_{2}$ and $\tau_{1}=\tau_{2}$. Furthermore, note that, for any complex-valued random variable $Z$, with complex conjugate $\bar{Z}$,

$$
\operatorname{Var}(\Re Z)=\frac{1}{2}(\operatorname{Var}(Z)+\Re \operatorname{Cov}(Z, \bar{Z})) ; \quad \operatorname{Var}(\Im Z)=\frac{1}{2}(\operatorname{Var}(Z)-\Re \operatorname{Cov}(Z, \bar{Z})),
$$

and we have $\overline{\mathbb{H}_{1,2}}=\mathbb{H}_{2,1}$.

\subsubsection{Pointwise Confidence Bands for $\mathfrak{R}$}

We utilize Theorem 3.4 to construct pointwise asymptotic $(1-\alpha)$-level confidence bands for the real and imaginary parts of $\mathfrak{R}^{j_{1}, j_{2}}\left(\omega ; \tau_{1}, \tau_{2}\right)$ as follows:

$$
C_{\mathrm{r}, n}^{(2)}\left(\omega_{k n} ; \tau_{1}, \tau_{2}\right):=\Re \hat{\mathfrak{R}}_{n, R}^{j_{1}, j_{2}}\left(\omega_{k n} ; \tau_{1}, \tau_{2}\right) \pm \Re \sigma_{(2)}^{j_{1}, j_{2}}\left(\omega_{k n} ; \tau_{1}, \tau_{2}\right) \Phi^{-1}(1-\alpha / 2),
$$

for the real part, and

$$
C_{\mathrm{i}, n}^{(2)}\left(\omega_{k n} ; \tau_{1}, \tau_{2}\right):=\Im \hat{\mathfrak{R}}_{n, R}^{j_{1}, j_{2}}\left(\omega_{k n} ; \tau_{1}, \tau_{2}\right) \pm \Im \sigma_{(2)}^{j_{1}, j_{2}}\left(\omega_{k n} ; \tau_{1}, \tau_{2}\right) \Phi^{-1}(1-\alpha / 2),
$$

for the imaginary part of the quantile coherency. Here, $\Phi$ stands for the cdf of the standard normal distribution,

$$
\left(\Re \sigma_{(2)}^{j_{1}, j_{2}}\left(\omega_{k n} ; \tau_{1}, \tau_{2}\right)\right)^{2}:=0 \vee \begin{cases}0 & \text { if } j_{1}=j_{2} \text { and } \tau_{1}=\tau_{2}, \\ \frac{1}{2}\left(\operatorname{Cov}\left(\mathbb{L}_{1,2}, \mathbb{L}_{1,2}\right)+\Re \operatorname{Cov}\left(\mathbb{L}_{1,2}, \mathbb{L}_{2,1}\right)\right) & \text { otherwise, }\end{cases}
$$

and

$$
\left(\Im \sigma_{(2)}^{j_{1}, j_{2}}\left(\omega_{k n} ; \tau_{1}, \tau_{2}\right)\right)^{2}:=0 \vee \begin{cases}0 & \text { if } j_{1}=j_{2} \text { and } \tau_{1}=\tau_{2}, \\ \frac{1}{2}\left(\operatorname{Cov}\left(\mathbb{L}_{1,2}, \mathbb{L}_{1,2}\right)-\Re \operatorname{Cov}\left(\mathbb{L}_{1,2}, \mathbb{L}_{2,1}\right)\right) & \text { otherwise. }\end{cases}
$$

The definition of $\sigma_{(2)}^{j_{1}, j_{2}}\left(\omega_{k n} ; \tau_{1}, \tau_{2}\right)$ is motivated by 25$)$ and the fact that we have $\overline{\mathbb{L}_{1,2}}=\mathbb{L}_{2,1}$. Furthermore, note that $\hat{\mathfrak{R}}_{n, R}^{j_{1}, j_{2}}\left(\omega_{k n} ; \tau_{1}, \tau_{2}\right)=1$, if $j_{1}=j_{2}$ and $\tau_{1}=\tau_{2}$..

In the definition of $\sigma_{(2)}^{j_{1}, j_{2}}\left(\omega_{k n} ; \tau_{1}, \tau_{2}\right)$ we have used $\operatorname{Cov}\left(\mathbb{L}_{a, b}, \mathbb{L}_{c, d}\right)$ to denote an estimator for

$$
\operatorname{Cov}\left(\mathbb{L}^{j_{1}, j_{2}}\left(\omega_{k n} ; \tau_{1}, \tau_{2}\right), \mathbb{L}^{j_{3}, j_{4}}\left(\omega_{k n} ; \tau_{3}, \tau_{4}\right)\right) .
$$


Recalling the definition of he limit process in Theorem 3.4 we derive the following expression:

$$
\begin{aligned}
& \frac{1}{\sqrt{\mathfrak{f}_{1,1} \mathfrak{f}_{2,2} \mathfrak{f}_{3,3} \mathfrak{f}_{4,4}}} \operatorname{Cov}\left(\mathbb{H}_{1,2}-\frac{1}{2} \frac{\mathfrak{f}_{1,2}}{\mathfrak{f}_{1,1}} \mathbb{H}_{1,1}-\frac{1}{2} \frac{\mathfrak{f}_{1,2}}{\mathfrak{f}_{2,2}} \mathbb{H}_{2,2}, \mathbb{H}_{3,4}-\frac{1}{2} \frac{\mathfrak{f}_{3,4}}{\mathfrak{f}_{3,3}} \mathbb{H}_{3,3}-\frac{1}{2} \frac{\mathfrak{f}_{3,4}}{\mathfrak{f}_{4,4}} \mathbb{H}_{4,4}\right) \\
& =\frac{\operatorname{Cov}\left(\mathbb{H}_{1,2}, \mathbb{H}_{3,4}\right)}{\sqrt{\mathfrak{f}_{1,1} \mathfrak{f}_{2,2} \mathfrak{f}_{3,3} \mathfrak{f}_{4,4}}}-\frac{1}{2} \frac{\overline{\mathfrak{f}_{3,4}} \operatorname{Cov}\left(\mathbb{H}_{1,2}, \mathbb{H}_{3,3}\right)}{\sqrt{\mathfrak{f}_{1,1} \mathfrak{f}_{2,2} \mathfrak{f}_{3,3}^{3} \mathfrak{f}_{4,4}}}-\frac{1}{2} \frac{\overline{\mathfrak{f}_{3,4}} \operatorname{Cov}\left(\mathbb{H}_{1,2}, \mathbb{H}_{4,4}\right)}{\sqrt{\mathfrak{f}_{1,1} \mathfrak{f}_{2,2} \mathfrak{f}_{3,3} \mathfrak{f}_{4,4}^{3}}} \\
& -\frac{1}{2} \frac{\mathfrak{f}_{1,2} \operatorname{Cov}\left(\mathbb{H}_{1,1}, \mathbb{H}_{3,4}\right)}{\sqrt{\mathfrak{f}_{1,1}^{3} \mathfrak{f}_{2,2} \mathfrak{f}_{3,3} \mathfrak{f}_{4,4}}}+\frac{1}{4} \frac{\mathfrak{f}_{1,2} \overline{\mathfrak{f}_{3,4}} \operatorname{Cov}\left(\mathbb{H}_{1,1}, \mathbb{H}_{3,3}\right)}{\sqrt{\mathfrak{f}_{1,1}^{3} \mathfrak{f}_{2,2} \mathfrak{f}_{3,3}^{3} \mathfrak{f}_{4,4}}}+\frac{1}{4} \frac{\mathfrak{f}_{1,2} \overline{\mathfrak{f}_{3,4}} \operatorname{Cov}\left(\mathbb{H}_{1,1}, \mathbb{H}_{4,4}\right)}{\sqrt{\mathfrak{f}_{1,1}^{3} \mathfrak{f}_{2,2} \mathfrak{f}_{3,3} \mathfrak{f}_{4,4}^{3}}} \\
& -\frac{1}{2} \frac{\mathfrak{f}_{1,2} \operatorname{Cov}\left(\mathbb{H}_{2,2}, \mathbb{H}_{3,4}\right)}{\sqrt{\mathfrak{f}_{1,1} \mathfrak{f}_{2,2}^{3} \mathfrak{f}_{3,3} \mathfrak{f}_{4,4}}}+\frac{1}{4} \frac{\mathfrak{f}_{1,2} \overline{\mathfrak{f}_{3,4}} \operatorname{Cov}\left(\mathbb{H}_{2,2}, \mathbb{H}_{3,3}\right)}{\sqrt{\mathfrak{f}_{1,1} \mathfrak{f}_{2,2}^{3} \mathfrak{f}_{3,3}^{3} \mathfrak{f}_{4,4}}}+\frac{1}{4} \frac{\mathfrak{f}_{1,2} \overline{\mathfrak{f}_{3,4}} \operatorname{Cov}\left(\mathbb{H}_{2,2}, \mathbb{H}_{4,4}\right)}{\sqrt{\mathfrak{f}_{1,1} \mathfrak{f}_{2,2}^{3} \mathfrak{f}_{3,3} \mathfrak{f}_{4,4}^{3}}},
\end{aligned}
$$

where we have written $\mathfrak{f}_{a, b}$ for the quantile spectral density $\mathfrak{f}^{j_{a}, j_{b}}\left(\omega_{k n} ; \tau_{a}, \tau_{b}\right)$, and $\mathbb{H}_{a, b}$ for the limit distribution $\mathbb{H}^{j_{a}, j_{b}}\left(\omega_{k n} ; \tau_{a}, \tau_{b}\right)$ for any $\left.a, b=1,2,3,4\right)$.

Thus, considering the special case where $\tau_{3}=\tau_{1}$ and $\tau_{4}=\tau_{2}$, we have

$$
\begin{aligned}
& \operatorname{Cov}\left(\mathbb{L}_{1,2}, \mathbb{L}_{1,2}\right) \\
& =\frac{1}{\mathfrak{f}_{1,1} \mathfrak{f}_{2,2}}\left(\operatorname{Cov}\left(\mathbb{H}_{1,2}, \mathbb{H}_{1,2}\right)-\Re \frac{\mathfrak{f}_{1,2} \operatorname{Cov}\left(\mathbb{H}_{1,1}, \mathbb{H}_{1,2}\right)}{\mathfrak{f}_{1,1}}-\Re \frac{\mathfrak{f}_{1,2} \operatorname{Cov}\left(\mathbb{H}_{2,2}, \mathbb{H}_{1,2}\right)}{\mathfrak{f}_{2,2}}\right. \\
& \left.\quad+\frac{1}{4}\left|\mathfrak{f}_{1,2}\right|^{2}\left(\frac{\operatorname{Cov}\left(\mathbb{H}_{1,1}, \mathbb{H}_{1,1}\right)}{\mathfrak{f}_{1,1}^{2}}+2 \Re \frac{\operatorname{Cov}\left(\mathbb{H}_{1,1}, \mathbb{H}_{2,2}\right)}{\mathfrak{f}_{1,1} \mathfrak{f}_{2,2}}+\frac{\operatorname{Cov}\left(\mathbb{H}_{2,2}, \mathbb{H}_{2,2}\right)}{\mathfrak{f}_{2,2}^{2}}\right)\right)
\end{aligned}
$$

and for the special case where $\tau_{3}=\tau_{1}$ and $\tau_{4}=\tau_{2}$ we have

$$
\begin{aligned}
& \operatorname{Cov}\left(\mathbb{L}_{1,2}, \mathbb{L}_{2,1}\right) \\
& =\frac{1}{\mathfrak{f}_{1,1} \mathfrak{f}_{2,2}}\left(\operatorname{Cov}\left(\mathbb{H}_{1,2}, \mathbb{H}_{2,1}\right)-\frac{\mathfrak{f}_{1,2} \operatorname{Cov}\left(\mathbb{H}_{1,2}, \mathbb{H}_{2,2}\right)}{\mathfrak{f}_{2,2}}-\frac{\mathfrak{f}_{1,2} \operatorname{Cov}\left(\mathbb{H}_{1,2}, \mathbb{H}_{1,1}\right)}{\mathfrak{f}_{1,1}}\right. \\
& \left.\quad+\frac{1}{4} \mathfrak{f}_{1,2}^{2}\left(\frac{\operatorname{Cov}\left(\mathbb{H}_{1,1}, \mathbb{H}_{1,1}\right)}{\mathfrak{f}_{1,1}^{2}}+2 \Re \frac{\operatorname{Cov}\left(\mathbb{H}_{1,1}, \mathbb{H}_{2,2}\right)}{\mathfrak{f}_{1,1} \mathfrak{f}_{2,2}}+\frac{\operatorname{Cov}\left(\mathbb{H}_{2,2}, \mathbb{H}_{2,2}\right)}{\mathfrak{f}_{2,2}^{2}}\right)\right) .
\end{aligned}
$$

We substitute consistent estimators for the unknown quantities. To do so we abuse notation using $\mathfrak{f}_{a, b}$ to denote $\tilde{G}_{n, R}^{j_{a}, j_{b}}\left(\omega_{k n} ; \tau_{a}, \tau_{b}\right)$ and write $\operatorname{Cov}\left(\mathbb{H}_{a, b}, \mathbb{H}_{c, d}\right)$ for the quantity defined in (24).

\subsection{Proof of Proposition 3.2}

The proof resembles the proof of Proposition 3.4 in Kley et al. (2015), where the univariate case was handled. For $j=1, \ldots, d$ we have, from the continuity of $F_{j}$ that the ranks of the random variables $X_{0, j}, \ldots, X_{n-1, j}$ and $F_{j}\left(X_{0, j}\right), \ldots, F_{j}\left(X_{n-1, j}\right)$ coincide almost surely. Thus, without loss of generality, we can assume that the CCR-periodogram is computed from the unobservable data $\left(F_{j}\left(X_{0, j}\right)\right)_{j=1, \ldots, d}, \ldots,\left(F_{j}\left(X_{n-1, j}\right)\right)_{j=1, \ldots, d}$. In particular, we can assume the marginals to be uniform. 
Applying the Continuous Mapping Theorem afterward, it suffices to prove

$$
\left(n^{-1 / 2} d_{n, R}^{j}(\omega ; \tau)\right)_{\tau \in[0,1], j=1, \ldots, d} \rightsquigarrow\left(\mathbb{D}^{j}(\omega ; \tau)\right)_{\tau \in[0,1], j=1, \ldots, d} \quad \text { in } \ell_{\mathbb{C}^{d}}^{\infty}([0,1]),
$$

where $\ell_{\mathbb{C}^{d}}^{\infty}([0,1])$ is the space of bounded functions $[0,1] \rightarrow \mathbb{C}^{d}$ that we identify with the product space $\ell^{\infty}([0,1])^{2 d}$. Let

$$
d_{n, U}^{j}(\omega ; \tau):=\sum_{t=0}^{n-1} I\left\{F_{j}\left(X_{t, j}\right) \leq \tau\right\} \mathrm{e}^{-\mathrm{i} \omega t}
$$

$j=1, \ldots, d, \omega \in \mathbb{R}, \tau \in[0,1]$, and note that for $(27)$ to hold, it is sufficient that

$$
\left(n^{-1 / 2} d_{n, U}^{j}(\omega ; \tau)\right)_{\tau \in[0,1], j=1, \ldots, d}
$$

satisfies the following two conditions:

(i1) convergence of the finite-dimensional distributions, i. e.,

$$
\left(n^{-1 / 2} d_{n, U}^{j_{\ell}}\left(\omega_{\ell} ; \tau_{\ell}\right)\right)_{\ell=1, \ldots, k} \stackrel{d}{\rightarrow}\left(\mathbb{D}^{j_{\ell}}\left(\omega_{\ell} ; \tau_{\ell}\right)\right)_{\ell=1, \ldots, k}
$$

for any $\left(j_{\ell}, \tau_{\ell}\right) \in\{1, \ldots, d\} \times[0,1], \omega_{\ell} \neq 0 \bmod 2 \pi, \ell=1, \ldots, k$ and $k \in \mathbb{N}$;

(i2) stochastic equicontinuity: for any $x>0$ and any $\omega \neq 0 \bmod 2 \pi$,

$$
\lim _{\delta \downarrow 0} \limsup _{n \rightarrow \infty} \mathbb{P}\left(\sup _{\substack{\tau_{1}, \tau_{2} \in[0,1] \\\left|\tau_{1}-\tau_{2}\right| \leq \delta}}\left|n^{-1 / 2}\left(d_{n, U}^{j}\left(\omega ; \tau_{1}\right)-d_{n, U}^{j}\left(\omega ; \tau_{2}\right)\right)\right|>x\right)=0, \quad \forall j=1, \ldots, d .
$$

Under (i1) and (i2), an application of Theorems 1.5.4 and 1.5.7 from van der Vaart and Wellner (1996) then yields

$$
\left(n^{-1 / 2} d_{n, U}^{j}(\omega ; \tau)\right)_{\tau \in[0,1], j=1, \ldots, d} \rightsquigarrow\left(\mathbb{D}^{j}(\omega ; \tau)\right)_{\tau \in[0,1], j=1, \ldots, d} \text { in } \ell_{\mathbb{C}^{d}}^{\infty}([0,1]) .
$$

In combination with

$$
\sup _{\tau \in[0,1]}\left|n^{-1 / 2}\left(d_{n, R}^{j}(\omega ; \tau)-d_{n, U}^{j}(\omega ; \tau)\right)\right|=o_{P}(1), \quad \text { for } \omega \neq 0 \quad \bmod 2 \pi, j=1, \ldots, d,
$$

which we will prove below, (30) yields the desired result: $(27)$. For the proof of $(31)$, we denote by $\hat{F}_{n, j}^{-1}(\tau):=\inf \left\{x: F_{n, j}(x) \geq \tau\right\}$ the generalized inverse of $\hat{F}_{n, j}$ and let inf $\emptyset:=0$. Then, we have, as in (7.25) of Kley et al. (2015), that

$$
\sup _{\omega \in \mathbb{R}} \sup _{\tau \in[0,1]}\left|d_{n, R}^{j}(\omega ; \tau)-d_{n, U}^{j}\left(\omega ; \hat{F}_{n, j}^{-1}(\tau)\right)\right| \leq n \sup _{\tau \in[0,1]}\left|\hat{F}_{n, j}(\tau)-\hat{F}_{n, j}(\tau-)\right|=O_{P}\left(n^{1 / 2 k}\right)
$$

where $\hat{F}_{n, j}(\tau-):=\lim _{\xi \uparrow 0} \hat{F}_{n, j}(\tau-\xi)$. The $O_{P}$-bound in 32 follows from Lemma 7.8 , Therefore, it suffices to bound the terms

$$
\left.\sup _{\tau \in[0,1]} n^{-1 / 2} \mid d_{n, U}^{j}\left(\omega ; \hat{F}_{n, j}^{-1}(\tau)\right)-d_{n, U}^{j}(\omega, \tau)\right) \mid, \text { for all } j=1, \ldots, d \text {. }
$$


To do so, note that, for any $x>0$ and $\delta_{n}=o(1)$ satisfying $n^{1 / 2} \delta_{n} \rightarrow \infty$, we have

$$
\begin{aligned}
& \left.\mathbb{P}\left(\sup _{\tau \in[0,1]} n^{-1 / 2} \mid d_{n, U}^{j}\left(\omega ; \hat{F}_{n, j}^{-1}(\tau)\right)-d_{n, U}^{j}(\omega ; \tau)\right) \mid>x\right) \\
& \leq \mathbb{P}\left(\sup _{\tau \in[0,1]} \sup _{|u-\tau| \leq \delta_{n}}\left|d_{n, U}^{j}(\omega ; u)-d_{n, U}^{j}(\omega ; \tau)\right|>x n^{1 / 2}, \sup _{\tau \in[0,1]}\left|\hat{F}_{n, j}^{-1}(\tau)-\tau\right| \leq \delta_{n}\right) \\
& \quad+\mathbb{P}\left(\sup _{\tau \in[0,1]}\left|\hat{F}_{n, j}^{-1}(\tau)-\tau\right|>\delta_{n}\right)=o(1)+o(1) .
\end{aligned}
$$

The first $o(1)$ follows from 29 . The second one is a consequence of Lemma 7.9.

It thus remains to prove (28) and (29). For any fixed $j=1, \ldots, d$ the process $\left(d_{n, U}^{j}(\omega, \tau)\right)_{\tau \in[0,1]}$ is determined by the univariate time series $X_{0, j}, \ldots, X_{n-1, j}$. Under the assumptions made here, (29) therefore follows from (8.7) in Kley et al. (2015).

Finally, we establish (28), by employing Lemma 7.7 in combination with Lemma P4.5 and Theorem 4.3.2 from Brillinger (1975). More precisely, to apply Lemma P4.5 (Brillinger, 1975), we have to verify that, for any $j_{1}, \ldots, j_{\ell} \in\{1, \ldots, d\}, \tau_{1}, \ldots, \tau_{\ell} \in[0,1], \ell \in \mathbb{N}$, and $\omega_{1}, \ldots, \omega_{\ell} \neq 0 \bmod 2 \pi$, all cumulants of the vector

$$
n^{-1 / 2}\left(d_{n, U}^{j_{1}}\left(\omega_{1} ; \tau_{1}\right), d_{n, U}^{j_{1}}\left(-\omega_{1} ; \tau_{1}\right), \ldots, d_{n, U}^{j_{\ell}}\left(\omega_{\ell} ; \tau_{\ell}\right), d_{n, U}^{j_{\ell}}\left(-\omega_{\ell} ; \tau_{\ell}\right)\right)
$$

converge to the corresponding cumulants of the vector

$$
\left(\mathbb{D}^{j_{1}}\left(\omega_{1} ; \tau_{1}\right), \mathbb{D}^{j_{1}}\left(-\omega_{1} ; \tau_{1}\right), \ldots, \mathbb{D}^{j_{\ell}}\left(\omega_{\ell} ; \tau_{\ell}\right), \mathbb{D}^{j_{\ell}}\left(-\omega_{\ell} ; \tau_{\ell}\right)\right) .
$$

For the cumulants of order one the arguments from the univariate case (cf. the proof of Proposition 3.4 in Kley et al. (2015)) apply: we have $\left|\mathbb{E}\left(n^{-1 / 2} d_{n, U}^{j}(\omega ; \tau)\right)\right|=o(1)$, for any $j=1, \ldots, d, \tau \in[0,1]$ and fixed $\omega \neq 0 \bmod 2 \pi$. Furthermore, for the cumulants of order two, applying Theorem 4.3.1 in Brillinger (1975) to the bivariate process $\left(I\left\{X_{t, j_{1}} \leq\right.\right.$ $\left.\left.q_{j_{1}}\left(\mu_{1}\right)\right\}, I\left\{X_{t, j_{2}} \leq q_{j_{2}}\left(\mu_{2}\right)\right\}\right)$, we obtain

$$
\operatorname{cum}\left(n^{-1 / 2} d_{n, U}^{i_{1}}\left(\lambda_{1} ; \mu_{1}\right), n^{-1 / 2} d_{n, U}^{i_{2}}\left(\lambda_{2} ; \mu_{2}\right)\right)=2 \pi n^{-1} \Delta_{n}\left(\lambda_{1}+\lambda_{2}\right) \mathfrak{f}^{i_{1}, i_{2}}\left(\lambda_{1} ; \mu_{1}, \mu_{2}\right)+o(1)
$$

for any $\left(i_{1}, \lambda_{1}, \mu_{1}\right),\left(i_{2}, \lambda_{2}, \mu_{2}\right) \in \bigcup_{\ell=1}^{k}\left\{\left(i_{\ell}, \omega_{\ell}, \tau_{\ell}\right),\left(j_{\ell},-\omega_{\ell}, \tau_{\ell}\right)\right\}$, which yields the correct second moment structure. The function $\Delta_{n}$ is defined in Lemma 7.7. Finally, the cumulants of order $J$, with $J \in \mathbb{N}$ and $J \geq 3$, all tend to zero, as in view of Lemma 7.7

$$
\begin{aligned}
& \operatorname{cum}\left(n^{-1 / 2} d_{n, U}^{i_{1}}\left(\lambda_{1} ; \mu_{1}\right), \ldots, n^{-1 / 2} d_{n, U}^{i_{J}}\left(\lambda_{J} ; \mu_{J}\right)\right) \\
& \quad \leq C n^{-J / 2}\left(\left|\Delta_{n}\left(\sum_{j=1}^{J} \lambda_{j}\right)\right|+1\right) \varepsilon(|\log \varepsilon|+1)^{d}=O\left(n^{-(J-2) / 2}\right)=o(1)
\end{aligned}
$$

for $\left(i_{1}, \lambda_{1}, \mu_{1}\right), \ldots,\left(i_{J}, \lambda_{J}, \mu_{J}\right) \in \bigcup_{\ell=1}^{k}\left\{\left(i_{\ell}, \omega_{\ell}, \tau_{\ell}\right),\left(i_{\ell},-\omega_{\ell}, \tau_{\ell}\right)\right\}$, where $\varepsilon:=\min _{j=1}^{J} \mu_{j}$. This implies that the limit $\mathbb{D}^{j}(\tau ; \omega)$ is Gaussian, and completes the proof of (28). Proposition 3.2 follows. 


\subsection{Proof of Theorem 3.3}

We proceed in a similar fashion as in the proof of the univariate estimator which was analyzed in Kley et al. (2015). First, we state an asymptotic representation result by which the estimator $\boldsymbol{G}_{n, R}$ can be approximated, in a suitable uniform sense, by another process $\hat{\boldsymbol{G}}_{n, U}$ which is not defined as a function of the standardized ranks $\hat{F}_{n, j}\left(X_{t, j}\right)$, but as a function of the unobservable quantities $F_{j}\left(X_{t, j}\right), t=0, \ldots, n-1, j=1, \ldots, d$. More precisely, this process is defined as

$$
\hat{\boldsymbol{G}}_{n, U}\left(\omega ; \tau_{1}, \tau_{2}\right):=\left(\hat{G}_{n, U}^{j_{1}, j_{2}}\left(\omega ; \tau_{1}, \tau_{2}\right)\right)_{j_{1}, j_{2}=1, \ldots, d},
$$

where

$$
\begin{aligned}
\hat{G}_{n, U}^{j_{1}, j_{2}}\left(\omega ; \tau_{1}, \tau_{2}\right) & :=\frac{2 \pi}{n} \sum_{s=1}^{n-1} W_{n}(\omega-2 \pi s / n) I_{n, U}^{j_{1}, j_{2}}\left(2 \pi s / n, \tau_{1}, \tau_{2}\right) \\
I_{n, U}^{j_{1}, j_{2}}\left(\omega ; \tau_{1}, \tau_{2}\right) & :=\frac{1}{2 \pi n} d_{n, U}^{j_{1}}\left(\omega ; \tau_{1}\right) d_{n, U}^{j_{2}}\left(-\omega ; \tau_{2}\right) \\
d_{n, U}^{j}(\omega ; \tau) & :=\sum_{t=0}^{n-1} I\left\{F_{j}\left(X_{t, j}\right) \leq \tau\right\} \mathrm{e}^{-\mathrm{i} \omega t} .
\end{aligned}
$$

Theorem 3.3 then follows from the asymptotic representation of $\hat{\boldsymbol{G}}_{n, R}$ by $\hat{\boldsymbol{G}}_{n, U}$ (i. e., Theorem $7.1($ iii) $)$ and the asymptotic properties of $\hat{\boldsymbol{G}}_{n, U}$ (i. e., Theorem 7.1(i)-(ii)), which we now state:

Theorem 7.1. Let Assumptions (C) and (W) hold, and assume that the distribution functions $F_{j}$ of $X_{0, j}$ are continuous for all $j=1, \ldots, d$. Let $b_{n}$ satisfy the assumptions of Theorem 3.3. Then,

(i) for any fixed $\omega \in \mathbb{R}$, as $n \rightarrow \infty$,

$$
\sqrt{n b_{n}}\left(\hat{\boldsymbol{G}}_{n, U}\left(\omega ; \tau_{1}, \tau_{2}\right)-\mathbb{E} \hat{\boldsymbol{G}}_{n, U}\left(\omega ; \tau_{1}, \tau_{2}\right)\right)_{\tau_{1}, \tau_{2} \in[0,1]} \rightsquigarrow \mathbb{H}(\omega ; \cdot, \cdot)
$$

in $\ell_{\mathbb{C}^{d \times d}}^{\infty}\left([0,1]^{2}\right)$, where the process $\mathbb{H}(\omega ; \cdot, \cdot)$ is defined in Theorem 3.3 ;

(ii) still as $n \rightarrow \infty$,

$$
\sup _{\substack{j_{1}, j_{2} \in\{1, \ldots, d\} \\ \tau_{1}, \tau_{2} \in[0,1] \\ \omega \in \mathbb{R}}}\left|\mathbb{E} \hat{G}_{n, U}^{j_{1}, j_{2}}\left(\tau_{1}, \tau_{2} ; \omega\right)-\mathfrak{f}^{j_{1}, j_{2}}\left(\omega ; \tau_{1}, \tau_{2}\right)-\left\{\boldsymbol{B}_{n}^{(k)}\left(\omega ; \tau_{1}, \tau_{2}\right)\right\}_{j_{1}, j_{2}}\right|=O\left(\left(n b_{n}\right)^{-1}\right)+o\left(b_{n}^{k}\right),
$$

where $\left\{\boldsymbol{B}_{n}^{(k)}\left(\omega ; \tau_{1}, \tau_{2}\right)\right\}_{j_{1}, j_{2}}$ is defined in 16 ;

(iii) for any fixed $\omega \in \mathbb{R}$,

$$
\sup _{\substack{j_{1}, j_{2} \in\{1, \ldots, d\} \\ \tau_{1}, \tau_{2} \in[0,1]}}\left|\hat{G}_{n, R}^{j_{1}, j_{2}}\left(\tau_{1}, \tau_{2} ; \omega\right)-\hat{G}_{n, U}^{j_{1}, j_{2}}\left(\tau_{1}, \tau_{2} ; \omega\right)\right|=o_{P}\left(\left(n b_{n}\right)^{-1 / 2}+b_{n}^{k}\right) ;
$$

if moreover the kernel $W$ is uniformly Lipschitz-continuous, this bound is uniform with respect to $\omega \in \mathbb{R}$. 
The proof of Theorem 7.1 is lengthy, technical and in many places similar to the proof of Theorem 3.6 in Kley et al. (2015). We provide the proof in Sections 7.3.1 7.3.3, with technical details deferred to Section 7.5. For the reader's convenience we first give a brief description of the necessary steps.

Part (ii) of Theorem 7.1 can be proved along the lines of classical results from Brillinger (1975), but uniformly with respect to the arguments $\tau_{1}$ and $\tau_{2}$. Parts (i) and (iii) require additional arguments that are different from the classical theory. These additional arguments are due to the fact that the estimator is a stochastic process and stochastic equicontinuity of

$$
\left(\hat{H}_{n}^{j_{1}, j_{2}}(a ; \omega)\right)_{a \in[0,1]^{2}}:=\sqrt{n b_{n}}\left(\hat{G}_{n, U}^{j_{1}, j_{2}}\left(\omega ; \tau_{1}, \tau_{2}\right)-\mathbb{E} \hat{G}_{n, U}^{j_{1}, j_{2}}\left(\omega ; \tau_{1}, \tau_{2}\right)\right)_{\tau_{1}, \tau_{2} \in[0,1]}
$$

for all $j_{1}, j_{2}=1, \ldots, d$ has to be proven to ensure that the convergence holds not only pointwise, but also uniformly. The key to the proof of (i) and (iii) is a uniform bound on the increments $\hat{H}_{n}^{j_{1}, j_{2}}(a ; \omega)-\hat{H}_{n}^{j_{1}, j_{2}}(b ; \omega)$ of the process $\hat{H}_{n}^{j_{1}, j_{2}}$. This bound is needed to show the stochastic equicontinuity of the process. To employ a restricted chaining technique (cf. Lemma 7.4), we require two different bounds. First, we prove a general bound, uniform in $a$ and $b$, on the moments of the increments $\hat{H}_{n}^{j_{1}, j_{2}}(a ; \omega)-\hat{H}_{n}^{j_{1}, j_{2}}(b ; \omega)$ (cf. Lemma 7.5). Second, we prove a sharper bound on the increments $\hat{H}_{n}^{j_{1}, j_{2}}(a ; \omega)-$ $\hat{H}_{n}^{j_{1}, j_{2}}(b ; \omega)$ when $a$ and $b$ are "sufficiently close" (cf. Lemma 7.11).

Condition (41) which we will required for Lemma 7.5 to hold is rather general. In Lemma 7.7 we prove that Assumption (C) implies (41).

\subsubsection{Proof of Theorem 7.1(i)}

It is sufficient to prove the following two claims:

(i1) convergence of the finite-dimensional distributions of the process (34), that is,

$$
\left(\hat{H}_{n}^{j_{1 \ell}, j_{2 \ell}}\left(\left(a_{1 \ell}, a_{2 \ell}\right) ; \omega_{j}\right)\right)_{j=1, \ldots, k} \stackrel{d}{\rightarrow}\left(\mathbb{H}^{j_{1 \ell}, j_{2 \ell}}\left(\left(a_{1 \ell}, a_{2 \ell}\right) ; \omega_{j}\right)\right)_{j=1, \ldots, k}
$$

for any $\left(j_{1 \ell}, j_{2 \ell}, a_{1 \ell}, a_{2 \ell}, \omega_{\ell}\right) \in\{1, \ldots, d\} \times[0,1]^{2} \times \mathbb{R}, \ell=1, \ldots, k$ and $k \in \mathbb{N}$;

(i2) stochastic equicontinuity: for any $x>0$, any $\omega \in \mathbb{R}$, and any $j_{1}, j_{2}=1, \ldots, d$,

$$
\lim _{\delta \downarrow 0} \limsup _{n \rightarrow \infty} \mathbb{P}\left(\sup _{\substack{a, b \in[0,1]^{2} \\\|a-b\|_{1} \leq \delta}}\left|\hat{H}_{n}^{j_{1}, j_{2}}(a ; \omega)-\hat{H}_{n}^{j_{1}, j_{2}}(b ; \omega)\right|>x\right)=0 .
$$

By (36) we have stochastic equicontinuity of all real parts $\Re \hat{H}_{n}^{j_{1}, j_{2}}(\cdot ; \omega)$ and imaginary parts $\Im H_{n}^{j_{1}, j_{2}}(\cdot ; \omega)$. Therefore, in view of Theorems 1.5.4 and 1.5.7 in van der Vaart and Wellner (1996), we will have proven part (i).

First we prove (i1). For fixed $\tau_{1}, \tau_{2}, \hat{G}_{n, U}^{j_{1}, j_{2}}\left(\omega ; \tau_{1}, \tau_{2}\right)$ is the traditional smoothed periodogram estimator of the cross-spectrum of the clipped processes $\left(I\left\{F_{j_{1}}\left(X_{t, j_{1}}\right) \leq \tau_{1}\right\}\right)_{t \in \mathbb{Z}}$ and $\left(I\left\{F_{j_{2}}\left(X_{t, j_{2}}\right) \leq \tau_{2}\right\}\right)_{t \in \mathbb{Z}}[$ see Chapter 7.1 in Brillinger (1975)]. Thus, (35) follows from Theorem 7.4.4 in Brillinger (1975), by which these estimators are asymptotically jointly Gaussian. The first and second moment structures of the limit are given by Theorem 7.4.1 
and Corollary 7.4.3 in Brillinger (1975). The joint convergence (35) follows. Note that condition (C) implies the summability condition [i. e., Assumption 2.6.2( $\ell$ ) in Brillinger (1975), for every $\ell]$ required for the three theorems in Brillinger $(1975)$ to be applied.

Now to the proof of (i2). The Orlicz norm $\|X\|_{\Psi}=\inf \{C>0: \mathbb{E} \Psi(|X| / C) \leq 1\}$ with $\Psi(x):=x^{6}$ coincides with the $L_{6}$ norm $\|X\|_{6}=\left(\mathbb{E}|X|^{6}\right)^{1 / 6}$. Therefore, for any $\kappa>0$ and sufficiently small $\|a-b\|_{1}$, we have by Lemma 7.5 and Lemma 7.7 that

$$
\left\|\hat{H}_{n}^{j_{1}, j_{2}}(a ; \omega)-\hat{H}_{n}^{j_{1}, j_{2}}(b ; \omega)\right\|_{\Psi} \leq K\left(\frac{\|a-b\|_{1}^{\kappa}}{\left(n b_{n}\right)^{2}}+\frac{\|a-b\|_{1}^{2 \kappa}}{n b_{n}}+\|a-b\|_{1}^{3 \kappa}\right)^{1 / 6} .
$$

Consequently, for all $a, b$ with $\|a-b\|_{1}$ sufficiently small and $\|a-b\|_{1} \geq\left(n b_{n}\right)^{-1 / \gamma}$ and all $\gamma \in(0,1)$ such that $\gamma<\kappa$,

$$
\left\|\hat{H}_{n}^{j_{1}, j_{2}}(a ; \omega)-\hat{H}_{n}^{j_{1}, j_{2}}(b ; \omega)\right\|_{\Psi} \leq \bar{K}\|a-b\|_{1}^{\gamma / 2} .
$$

Note that $\|a-b\|_{1} \geq\left(n b_{n}\right)^{-1 / \gamma}$ if and only if $d(a, b):=\|a-b\|_{1}^{\gamma / 2} \geq\left(n b_{n}\right)^{-1 / 2}=: \bar{\eta}_{n} / 2$. The packing number (van der Vaart and Wellner, 1996, p. 98) $D(\varepsilon, d)$ of $\left([0,1]^{2}, d\right)$ satisfies $D(\varepsilon, d) \asymp \varepsilon^{-4 / \gamma}$. By Lemma 7.4. we therefore have, for all $x, \delta>0$ and $\eta \geq \bar{\eta}_{n}$,

$$
\begin{aligned}
& \mathbb{P}\left(\sup _{\|a-b\|_{1} \leq \delta^{2 / \gamma}}\left|\hat{H}_{n}^{j_{1}, j_{2}}(a ; \omega)-\hat{H}_{n}^{j_{1}, j_{2}}(b ; \omega)\right|>x\right) \\
= & \mathbb{P}\left(\sup _{d(a, b) \leq \delta}\left|\hat{H}_{n}^{j_{1}, j_{2}}(a ; \omega)-\hat{H}_{n}^{j_{1}, j_{2}}(b ; \omega)\right|>x\right) \\
\leq & {\left[\frac{8 \tilde{K}}{x}\left(\int_{\bar{\eta}_{n} / 2}^{\eta} \epsilon^{-2 /(3 \gamma)} \mathrm{d} \epsilon+\left(\delta+2 \bar{\eta}_{n}\right) \eta^{-4 /(3 \gamma)}\right)\right]^{6} } \\
& +\mathbb{P}\left(\sup _{d(a, b) \leq \bar{\eta}_{n}}\left|\hat{H}_{n}^{j_{1}, j_{2}}(a ; \omega)-\hat{H}_{n}^{j_{1}, j_{2}}(b ; \omega)\right|>x / 4\right) .
\end{aligned}
$$

Now, choosing $2 / 3<\gamma<1$ and letting $n$ tend to infinity, the second term tends to zero by Lemma 7.11, because, by construction, $1 / \gamma>1$ and $d(a, b) \leq \bar{\eta}_{n}$ if and only if $\|a-b\|_{1} \leq 2^{2 / \gamma}\left(n b_{n}\right)^{-1 / \gamma}$. All together, this yields

$$
\lim _{\delta \downarrow 0} \limsup _{n \rightarrow \infty} \mathbb{P}\left(\sup _{d(a, b) \leq \delta}\left|\hat{H}_{n}(a ; \omega)-\hat{H}_{n}(b ; \omega)\right|>x\right) \leq\left[\frac{8 \tilde{K}}{x} \int_{0}^{\eta} \epsilon^{-2 /(3 \gamma)} \mathrm{d} \epsilon\right]^{6},
$$

for every $x, \eta>0$. The claim then follows, as the integral on the right-hand side may be arbitrarily small by choosing $\eta$ accordingly.

\subsubsection{Proof of Theorem 7.1(ii)}

Following the arguments which were applied in Section 8.1 of Kley et al. (2015) we can derive asymptotic expansions for $\mathbb{E}\left[I_{n, U}^{j_{1}, j_{2}}\left(\omega ; \tau_{1}, \tau_{2}\right)\right]$ and $\mathbb{E}\left[\hat{G}_{n, U}^{j_{1}, j_{2}}\left(\omega ; \tau_{1}, \tau_{2}\right)\right]$. In fact, it is easy to see that the proofs can still be applied when the Laplace cumulants

$$
\operatorname{cum}\left(I\left\{X_{k_{1}} \leq x_{1}\right\}, I\left\{X_{k_{2}} \leq x_{2}\right\}, \ldots, I\left\{X_{0} \leq x_{p}\right\}\right)
$$


which were considered in Kley et al. (2015) are replaced by their multivariate counterparts

$$
\operatorname{cum}\left(I\left\{X_{k_{1}, j_{1}} \leq x_{1}\right\}, I\left\{X_{k_{2}, j_{2}} \leq x_{2}\right\}, \ldots, I\left\{X_{0, j_{p}} \leq x_{p}\right\}\right)
$$

More precisely, we now state Lemma 7.2 and 7.3 (without proof) that are multivariate counterparts to Lemmas 8.4 and 8.5 in Kley et al. (2015). The lemmas hold under the following assumption:

(CS) Let $p \geq 2, \delta>0$. There exists a non-increasing function $a_{p}: \mathbb{N} \rightarrow \mathbb{R}^{+}$such that

$$
\sum_{k \in \mathbb{N}} k^{\delta} a_{p}(k)<\infty
$$

and

$$
\sup _{x_{1}, \ldots, x_{p}}\left|\operatorname{cum}\left(I\left\{X_{k_{1}, j_{1}} \leq x_{1}\right\}, I\left\{X_{k_{2}, j_{2}} \leq x_{2}\right\}, \ldots, I\left\{X_{0, j_{p}} \leq x_{p}\right\}\right)\right| \leq a_{p}\left(\max _{j}\left|k_{j}\right|\right),
$$

for all $j_{1}, \ldots, j_{p}=1, \ldots, d$.

Note that condition (CS) follows from Assumption (C) but is in fact somewhat weaker. We now state the first of the two lemmas. It is a generalization of Theorem 5.2.2 in Brillinger (1975).

Lemma 7.2. Under (CS) with $K=2, \delta>3$,

$$
\mathbb{E} I_{n, U}^{j_{1}, j_{2}}\left(\omega ; \tau_{1}, \tau_{2}\right)=\left\{\begin{array}{lll}
\mathfrak{f}^{j_{1}, j_{2}}\left(\omega ; \tau_{1}, \tau_{2}\right)+\frac{1}{2 \pi n}\left[\frac{\sin (n \omega / 2)}{\sin (\omega / 2)}\right]^{2} \tau_{1} \tau_{2}+\varepsilon_{n}^{\tau_{1}, \tau_{2}}(\omega) & \omega \neq 0 & \bmod 2 \pi \\
\mathfrak{f}^{j_{1}, j_{2}}\left(\omega ; \tau_{1}, \tau_{2}\right)+\frac{n}{2 \pi} \tau_{1} \tau_{2}+\varepsilon_{n}^{\tau_{1}, \tau_{2}}(\omega) & \omega=0 & \bmod 2 \pi
\end{array}\right.
$$

with $\sup _{\tau_{1}, \tau_{2} \in[0,1], \omega \in \mathbb{R}}\left|\varepsilon_{n}^{\tau_{1}, \tau_{2}}(\omega)\right|=O(1 / n)$.

The second of the two lemmas is a generalization of Theorem 5.6.1 in Brillinger (1975).

Lemma 7.3. Assume that (CS), with $p=2$ and $\delta>k+1$, and (W) hold. Then, with the notation of Theorem 3.3 ,

$\sup _{\tau_{1}, \tau_{2} \in[0,1], \omega \in \mathbb{R}}\left|\mathbb{E} \hat{G}_{n}^{j_{1}, j_{2}}\left(\omega ; \tau_{1}, \tau_{2}\right)-\mathfrak{f}^{j_{1}, j_{2}}\left(\omega ; \tau_{1}, \tau_{2}\right)-\left\{\boldsymbol{B}_{n}^{(k)}\left(\omega ; \tau_{1}, \tau_{2}\right)\right\}_{j_{1}, j_{2}}\right|=O\left(\left(n b_{n}\right)^{-1}\right)+o\left(b_{n}^{k}\right)$.

Because (C) implies (CS), Lemma 7.3 implies Theorem 7.1(ii).

\subsubsection{Proof of Theorem 7.1(iii)}

Using (31) and argument similar to the ones in the proof of Lemma 7.11 it follows that

$$
\sup _{\omega \in \mathbb{R}} \sup _{\tau_{1}, \tau_{2} \in[0,1]}\left|\hat{G}_{n, R}^{j_{1}, j_{2}}\left(\omega ; \tau_{1}, \tau_{2}\right)-\hat{G}_{n, U}^{j_{1}, j_{2}}\left(\omega ; \hat{F}_{n, j_{1}}^{-1}\left(\tau_{1}\right), \hat{F}_{n, j_{2}}^{-1}\left(\tau_{2}\right)\right)\right|=o_{P}(1) .
$$


It therefore suffices to bound the differences

$$
\sup _{\tau_{1}, \tau_{2} \in[0,1]}\left|\hat{G}_{n, U}^{j_{1}, j_{2}}\left(\omega ; \tau_{1}, \tau_{2}\right)-\hat{G}_{n, U}^{j_{1}, j_{2}}\left(\omega ; \hat{F}_{n, j_{1}}^{-1}\left(\tau_{1}\right), \hat{F}_{n, j_{2}}^{-1}\left(\tau_{2}\right)\right)\right|
$$

for $j_{1}, j_{2}=1, \ldots, d$, pointwise and uniformly in $\omega$.

We first prove the statement for fixed $\omega \in \mathbb{R}$ in full details and will later sketch the additional arguments needed for the proof of the uniform result. For any $x>0$ and sequence $\delta_{n}$ we have,

$$
\begin{aligned}
& P^{n}(\omega):=\mathbb{P}\left(\sup _{\tau_{1}, \tau_{2} \in[0,1]}\left|\hat{G}_{n, U}^{j_{1}, j_{2}}\left(\omega ; \hat{F}_{n, j_{1}}^{-1}\left(\tau_{1}\right), \hat{F}_{n, j_{2}}^{-1}\left(\tau_{2}\right)\right)-\hat{G}_{n, U}^{j_{1}, j_{2}}\left(\omega ; \tau_{1}, \tau_{2}\right)\right|>x\left(\left(n b_{n}\right)^{-1 / 2}+b_{n}^{k}\right)\right) \\
& \leq \mathbb{P}\left(\sup _{\tau_{1}, \tau_{2} \in[0,1]} \sup _{\substack{\left\|(u, v)-\left(\tau_{1}, \tau_{2}\right)\right\|_{\infty} \\
\leq \sup _{i=1,2 ; \tau \in[0,1]} \hat{F}_{n, j_{i}}^{-1}(\tau)-\tau \mid}}\left|\hat{G}_{n, U}^{j_{1}, j_{2}}(\omega ; u, v)-\hat{G}_{n, U}^{j_{1}, j_{2}}\left(\omega ; \tau_{1}, \tau_{2}\right)\right|>x\left(\left(n b_{n}\right)^{-1 / 2}+b_{n}^{k}\right)\right) \\
& \leq \mathbb{P}\left(\sup _{\tau_{1}, \tau_{2} \in[0,1]} \sup _{\substack{\left|u-\tau_{1}\right| \leq \delta_{n} \\
\left|v-\tau_{2}\right| \leq \delta_{n}}}\left|\hat{G}_{n, U}^{j_{1}, j_{2}}(\omega ; u, v)-\hat{G}_{n, U}^{j_{1}, j_{2}}\left(\omega ; \tau_{1}, \tau_{2}\right)\right|>x\left(\left(n b_{n}\right)^{-1 / 2}+b_{n}^{k}\right),\right. \\
& \left.\sup _{i=1,2 ; \tau \in[0,1]}\left|\hat{F}_{n, j_{i}}^{-1}(\tau)-\tau\right| \leq \delta_{n}\right)+\sum_{i=1}^{2} \mathbb{P}\left(\sup _{\tau \in[0,1]}\left|\hat{F}_{n, j_{i}}^{-1}(\tau)-\tau\right|>\delta_{n}\right) \\
& =P_{1}^{n}+P_{2}^{n} \text {, say. }
\end{aligned}
$$

We choose $\delta_{n}$ such that $n^{-1 / 2} \ll \delta_{n}=o\left(n^{-1 / 2} b_{n}^{-1 / 2}(\log n)^{-D}\right)$, where $D$ denotes the constant from Lemma 7.6. It then follows from Lemma 7.9 that $P_{2}^{n}$ is $o(1)$. For $P_{1}^{n}$, on the other hand, we have the following bound:

$$
\begin{aligned}
& \mathbb{P}\left(\sup _{\tau_{1}, \tau_{2} \in[0,1]} \sup _{\left|u-\tau_{1}\right| \leq \delta_{n}}\left|\hat{H}_{n, U}^{j_{1}, j_{2}}(\omega ; u, v)-\hat{H}_{n, U}^{j_{1}, j_{2}}\left(\omega ; \tau_{1}, \tau_{2}\right)\right|>\left(1+\left(n b_{n}\right)^{1 / 2} b_{n}^{k}\right) x / 2\right) \\
& \quad+I\left\{\sup _{\tau_{1}, \tau_{2} \in[0,1]} \sup _{\substack{u-\tau_{1}\left|\leq \delta_{n}\\
\right| v-\tau_{2} \mid \leq \delta_{n}}}\left|\mathbb{E} \hat{G}_{n, U}^{j_{1}, j_{2}}(\omega ; u, v)-\mathbb{E} \hat{G}_{n, U}^{j_{1}, j_{2}}\left(\omega ; \tau_{1}, \tau_{2}\right)\right|>\left(\left(n b_{n}\right)^{-1 / 2}+b_{n}^{k}\right) x / 2\right\} .
\end{aligned}
$$

The first term tends to zero because of (36). The indicator vanishes for $n$ large enough, because we have

$$
\begin{aligned}
& \sup _{\tau_{1}, \tau_{2} \in[0,1]} \sup _{\substack{\left|u-\tau_{1}\right| \leq \delta_{n} \\
\left|v-\tau_{2}\right| \leq \delta_{n}}}\left|\mathbb{E} \hat{G}_{n, U}^{j_{1}, j_{2}}(\omega ; u, v)-\mathbb{E} \hat{G}_{n, U}^{j_{1}, j_{2}}\left(\omega ; \tau_{1}, \tau_{2}\right)\right| \\
& \leq \sup _{\tau_{1}, \tau_{2} \in[0,1]} \sup _{\left|u-\tau_{1}\right| \leq \delta_{n}}\left|\mathbb{E} \hat{G}_{n, U}^{j_{1}, j_{2}}(\omega ; u, v)-\mathfrak{f}^{j_{1}, j_{2}}(\omega ; u, v)-\left\{B_{n}^{(k)}(\omega ; u, v)\right\}_{j_{1}, j_{2}}\right| \\
& \left.+\sup _{\tau_{1}, \tau_{2} \in[0,1]} \sup _{\left|u-\tau_{1}\right| \leq \delta_{n}} \mid v B_{n}^{(k)}\left(\omega ; \tau_{1}, \tau_{2}\right)\right\}_{j_{1}, j_{2}}+\mathfrak{f}^{j_{1}, j_{2}}\left(\omega ; \tau_{1}, \tau_{2}\right)-\mathbb{E} \hat{G}_{n, U}^{j_{1}, j_{2}}\left(\omega ; \tau_{1}, \tau_{2}\right) \mid \\
& +\sup _{\tau_{1}, \tau_{2} \in[0,1]} \sup _{\substack{\left|u-\tau_{1}\right| \leq \delta_{n} \\
\left|v-\tau_{2}\right| \leq \delta_{n}}} \mid \mathfrak{f}^{j_{1}, j_{2}}(\omega ; u, v)+\left\{B_{n}^{(k)}(\omega ; u, v)\right\}_{j_{1}, j_{2}} \\
& -\mathfrak{f}^{j_{1}, j_{2}}\left(\omega ; \tau_{1}, \tau_{2}\right)-\left\{B_{n}^{(k)}\left(\omega ; \tau_{1}, \tau_{2}\right)\right\}_{j_{1}, j_{2}} \mid \\
& =o\left(n^{-1 / 2} b_{n}^{-1 / 2}+b_{n}^{k}\right)+O\left(\delta_{n}\left(1+\left|\log \delta_{n}\right|\right)^{D}\right) \text {, }
\end{aligned}
$$


where $D$ is still the constant from Lemma 7.6. To bound the first two terms we have applied part (ii) of Theorem 7.1 and Lemma 7.6 for the third one. Thus, for any fixed $\omega$, we have shown $P^{n}(\omega)=o(1)$, which is the pointwise version of the claim.

Next, we outline the proof of the uniform (with respect to $\omega$ ) convergence. For any $y_{n}>0$, by similar arguments as above, using the same $\delta_{n}$, we have

$$
\begin{aligned}
& \mathbb{P}\left(\sup _{\omega \in \mathbb{R}} \sup _{\tau_{1}, \tau_{2} \in[0,1]}\left|\hat{G}_{n, R}^{j_{1}, j_{2}}\left(\omega ; \tau_{1}, \tau_{2}\right)-\hat{G}_{n, U}^{j_{1}, j_{2}}\left(\omega ; \tau_{1}, \tau_{2}\right)\right|>y_{n}\right) \\
& \leq \mathbb{P}\left(\sup _{\omega \in \mathbb{R}} \sup _{\tau_{1}, \tau_{2} \in[0,1]} \sup _{\left|u-\tau_{1}\right| \leq \delta_{n}}\left|\hat{H}_{n, U}^{j_{1}, j_{2}}(\omega ; u, v)-\hat{H}_{n, U}^{j_{1}, j_{2}}\left(\omega ; \tau_{1}, \tau_{2}\right)\right|>\left(n b_{n}\right)^{1 / 2} y_{n} / 2\right) \\
& +I\left\{\sup _{\omega \in \mathbb{R}} \sup _{\tau_{1}, \tau_{2} \in[0,1]} \sup _{\substack{\left|u-\tau_{1}\right| \leq \delta_{n} \\
\left|v-\tau_{2}\right| \leq \delta_{n}}}\left|\mathbb{E} \hat{G}_{n, U}^{j_{1}, j_{2}}(\omega ; u, v)-\mathbb{E} \hat{G}_{n, U}^{j_{1}, j_{2}}\left(\omega ; \tau_{1}, \tau_{2}\right)\right|>y_{n} / 2\right\}+o(1) .
\end{aligned}
$$

The indicator in the latter expression is $o(1)$ by the same arguments as above [note that Lemma 7.6 and the statement of part (ii) both hold uniformly with respect to $\omega \in \mathbb{R}]$. For the bound of the probability, note that by Lemma 7.10 ,

$$
\sup _{\tau_{1}, \tau_{2}} \sup _{k=1, \ldots, n}\left|I_{n, U}^{j_{1}, j_{2}}\left(2 \pi k / n ; \tau_{1}, \tau_{2}\right)\right|=O_{P}\left(n^{2 / K}\right) \text {, for any } K>0 .
$$

Moreover, by the uniform Lipschitz continuity of $W$ the function $W_{n}$ is also uniformly Lipschitz continuous with constant of order $O\left(b_{n}^{-2}\right)$. Combining those facts with Lemma 7.6 and the assumptions on $b_{n}$, we obtain

$$
\sup _{\substack{\omega_{1}, \omega_{2} \in \mathbb{R} \\\left|\omega_{1}-\omega_{2}\right| \leq n^{-3}}} \sup _{\tau_{1}, \tau_{2} \in[0,1]}\left|\hat{H}_{n, U}^{j_{1}, j_{2}}\left(\omega_{1} ; \tau_{1}, \tau_{2}\right)-\hat{H}_{n, U}^{j_{1}, j_{2}}\left(\omega_{2} ; \tau_{1}, \tau_{2}\right)\right|=o_{P}(1) .
$$

By the periodicity of $\hat{H}_{n, U}^{j_{1}, j_{2}}$ (with respect to $\omega$ ), it suffices to show that

$$
\max _{\omega=0,2 \pi n^{-3}, \ldots, 2 \pi} \sup _{\tau_{1}, \tau_{2} \in[0,1]} \sup _{\substack{\left|u-\tau_{1}\right| \leq \delta_{n} \\\left|v-\tau_{2}\right| \leq \delta_{n}}}\left|\hat{H}_{n, U}^{j_{1}, j_{2}}(\omega ; u, v)-\hat{H}_{n, U}^{j_{1}, j_{2}}\left(\omega ; \tau_{1}, \tau_{2}\right)\right|=o_{P}(1) .
$$

By Lemmas 7.4 and 7.11 there exists a random variable $S(\omega)$ such that

$$
\sup _{\tau_{1}, \tau_{2} \in[0,1]} \sup _{\substack{\left|u-\tau_{1}\right| \leq \delta_{n} \\\left|v-\tau_{2}\right| \leq \delta_{n}}}\left|\hat{H}_{n, U}^{j_{1}, j_{2}}(\omega ; u, v)-\hat{H}_{n, U}^{j_{1}, j_{2}}\left(\omega ; \tau_{1}, \tau_{2}\right)\right| \leq|S(\omega)|+R_{n}(\omega),
$$

for any fixed $\omega \in \mathbb{R}$, with $\sup _{\omega \in \mathbb{R}}\left|R_{n}(\omega)\right|=o_{P}(1)$ and

$$
\max _{\omega=0,2 \pi n^{-3} \ldots, 2 \pi} \mathbb{E}\left[\left|S^{2 L}(\omega)\right|\right] \leq K_{L}^{2 L}\left(\int_{0}^{\eta} \epsilon^{-4 /(2 L \gamma)} \mathrm{d} \epsilon+\left(\delta_{n}^{\gamma / 2}+2\left(n b_{n}\right)^{-1 / 2}\right) \eta^{-8 /(2 L \gamma)}\right)^{2 L}
$$

for any $0<\gamma<1, L \in \mathbb{N}, 0<\eta<\delta_{n}$, and a constant $K_{L}$ depending on $L$ only. For appropriately chosen $L$ and $\gamma$, this latter bound is $o\left(n^{-3}\right)$. Note that the maximum is with respect to a set of cardinality $O\left(n^{3}\right)$, which completes the proof of part (iii). 


\subsection{Proof of Theorem 3.4}

By a Taylor expansion we have, for every $x, x_{0}>0$,

$$
\frac{1}{\sqrt{x}}=\frac{1}{\sqrt{x_{0}}}-\frac{1}{2} \frac{1}{\sqrt{x_{0}^{3}}}\left(x-x_{0}\right)+\frac{3}{8} \xi_{x, x_{0}}^{-5 / 2}\left(x-x_{0}\right)^{2},
$$

where $\xi_{x, x_{0}}$ is between $x$ and $x_{0}$. Let $R_{n}\left(x, x_{0}\right):=\frac{3}{8} \xi_{x, x_{0}}^{-5 / 2}\left(x-x_{0}\right)^{2}$, then

$$
\frac{x}{\sqrt{y z}}-\frac{x_{0}}{\sqrt{y_{0} z_{0}}}=\frac{1}{\sqrt{y_{0} z_{0}}}\left(\left(x-x_{0}\right)-\frac{1}{2} \frac{x_{0}}{y_{0}}\left(y-y_{0}\right)-\frac{1}{2} \frac{x_{0}}{z_{0}}\left(z-z_{0}\right)\right)+r_{n},
$$

where

$$
\begin{aligned}
r_{n}= & \left(x-x_{0}\right)\left(-\frac{1}{2} \frac{1}{y_{0}}\left(y-y_{0}\right)-\frac{1}{2} \frac{1}{z_{0}}\left(z-z_{0}\right)\right) \\
+ & x\left(R_{n}\left(y, y_{0}\right) \sqrt{y_{0}}\left(1-\frac{1}{2} \frac{1}{z_{0}}\left(z-z_{0}\right)\right)+R_{n}\left(z, z_{0}\right) \sqrt{z_{0}}\left(1-\frac{1}{2} \frac{1}{y_{0}}\left(y-y_{0}\right)\right)\right. \\
& \left.+\frac{1}{4} \frac{1}{y_{0}}\left(y-y_{0}\right) \frac{1}{z_{0}}\left(z-z_{0}\right)+\sqrt{y_{0} z_{0}} R_{n}\left(y, y_{0}\right) R_{n}\left(z, z_{0}\right)\right)
\end{aligned}
$$

Write $\mathfrak{f}_{a, b}$ for $\mathfrak{f}^{j_{a}, j_{b}}\left(\omega ; \tau_{a}, \tau_{b}\right), \mathfrak{G}_{a, b}$ for $\hat{G}_{n, R}^{j_{a}, j_{b}}\left(\omega ; \tau_{a}, \tau_{b}\right)$, and $\boldsymbol{B}_{a, b}$ for $\left\{\boldsymbol{B}_{n}^{(k)}\left(\omega ; \tau_{a}, \tau_{b}\right)\right\}_{j_{a}, j_{b}}$ $(a, b=1,2,3,4)$. We want to employ $(38)$ and to this end let

$$
\begin{aligned}
& x:=\mathfrak{G}_{a, b} \quad y:=\mathfrak{G}_{a, a} \quad z:=\mathfrak{G}_{b, b} \\
& x_{0}:=\mathfrak{f}_{a, b}+\boldsymbol{B}_{a, b} \quad y_{0}:=\mathfrak{f}_{a, a}+\boldsymbol{B}_{a, a} \quad z_{0}:=\mathfrak{f}_{b, b}+\boldsymbol{B}_{b, b}
\end{aligned}
$$

By Theorem 3.3 the differences $x-x_{0}, y-y_{0}$, and $z-z_{0}$ are in $O_{P}\left(\left(n b_{n}\right)^{-1 / 2}\right)$, uniformly with respect to $\tau_{1}, \tau_{2}$. Under the assumption that $n b_{n} \rightarrow \infty$, as $n \rightarrow \infty$, this entails $\mathfrak{G}_{a, a}-\boldsymbol{B}_{a, a} \rightarrow \mathfrak{f}_{a, a}$, in probability. For $\varepsilon \leq \tau_{1}, \tau_{2} \leq 1-\varepsilon$, we have $\mathfrak{f}_{a, a}>0$, such that, by the Continuous Mapping Theorem we have $\left(\mathfrak{G}_{a, a}-\boldsymbol{B}_{a, a}\right)^{-5 / 2} \rightarrow \mathfrak{f}_{a, a}^{-5 / 2}$, in probability. As $\boldsymbol{B}_{a, a}=o(1)$, we have $y^{-5 / 2}-y_{0}^{-5 / 2}=o_{P}(1)$. Finally, due to

$$
\xi_{y, y_{0}}^{-5 / 2} \leq y_{n}^{-5 / 2} \vee y_{0}^{-5 / 2} \leq\left(y_{n}^{-5 / 2}-y_{0}^{-5 / 2}\right) \vee 0+y_{0}^{-5 / 2}=o_{P}(1)+O(1)=O_{P}(1),
$$

we have that $R_{n}\left(y, y_{0}\right)=O_{P}\left(\left(n b_{n}\right)^{-1}\right)$. Analogous arguments yields $R_{n}\left(z, z_{0}\right)=O_{P}\left(\left(n b_{n}\right)^{-1}\right)$. Thus we have shown that

$$
\begin{aligned}
& \hat{\mathfrak{R}}_{n, R}^{j_{1}, j_{2}}\left(\omega ; \tau_{1}, \tau_{2}\right)-\frac{\mathfrak{f}_{a, b}+\boldsymbol{B}_{a, b}}{\sqrt{\mathfrak{f}_{a, a}+\boldsymbol{B}_{a, a}} \sqrt{\mathfrak{f}_{b, b}+\boldsymbol{B}_{b, b}}} \\
& \begin{array}{r}
\frac{1}{\sqrt{\mathfrak{f}_{1,1} \mathfrak{f}_{2,2}}}\left(\left[\mathfrak{G}_{1,2}-\mathfrak{f}_{1,2}-\boldsymbol{B}_{1,2}\right]-\frac{1}{2} \frac{\mathfrak{f}_{1,2}}{\mathfrak{f}_{1,1}}\left[\mathfrak{G}_{1,1}-\mathfrak{f}_{1,1}-\boldsymbol{B}_{1,1}\right]-\frac{1}{2} \frac{\mathfrak{f}_{1,2}}{\mathfrak{\mathfrak { f }}_{2,2}}\left[\mathfrak{G}_{2,2}-\mathfrak{f}_{2,2}-\boldsymbol{B}_{2,2}\right]\right) \\
+O_{P}\left(1 /\left(n b_{n}\right)\right),
\end{array}
\end{aligned}
$$

with the $O_{P}$ holding uniformly with respect to $\tau_{1}, \tau_{2}$. Further more, note that

$$
\begin{aligned}
& \frac{\mathfrak{f}_{a, b}+\boldsymbol{B}_{a, b}}{\sqrt{\mathfrak{f}_{a, a}+\boldsymbol{B}_{a, a}} \sqrt{\mathfrak{f}_{b, b}+\boldsymbol{B}_{b, b}}}= \frac{\mathfrak{f}_{a, b}}{\sqrt{\mathfrak{f}_{a, a} \mathfrak{f}_{b, b}}}+\frac{1}{\sqrt{\mathfrak{f}_{a, a} \mathfrak{f}_{b, b}}}\left(\boldsymbol{B}_{a, b}-\frac{1}{2} \frac{\mathfrak{f}_{a, b}}{\mathfrak{f}_{a, a}} \boldsymbol{B}_{a, a}-\frac{1}{2} \frac{\mathfrak{f}_{a, b}}{\mathfrak{f}_{b, b}} \boldsymbol{B}_{b, b}\right) \\
&+O\left(\left|\boldsymbol{B}_{a, b}\right|\left(\boldsymbol{B}_{a, a}+\boldsymbol{B}_{b, b}\right)+\boldsymbol{B}_{a, a}^{2}+\boldsymbol{B}_{b, b}^{2}+\boldsymbol{B}_{a, a} \boldsymbol{B}_{b, b}\right),
\end{aligned}
$$


where we have used (38) again. Condition (19) implies that the remainder is of order $o\left(\left(n b_{n}\right)^{-1 / 2}\right)$. Therefore,

$$
\sqrt{n b_{n}}\left(\hat{\mathfrak{R}}_{n, R}^{j_{1}, j_{2}}\left(\omega ; \tau_{1}, \tau_{2}\right)-\mathfrak{R}^{j_{1}, j_{2}}\left(\omega ; \tau_{1}, \tau_{2}\right)-\frac{1}{\sqrt{\mathfrak{f}_{a, a} \mathfrak{f}_{b, b}}}\left(\boldsymbol{B}_{a, b}-\frac{1}{2} \frac{\mathfrak{f}_{a, b}}{\mathfrak{f}_{a, a}} \boldsymbol{B}_{a, a}-\frac{1}{2} \frac{\mathfrak{f}_{a, b}}{\mathfrak{f}_{b, b}} \boldsymbol{B}_{b, b}\right)\right)_{\tau_{1}, \tau_{2} \in[0,1]}
$$

and

$$
\frac{\sqrt{n b_{n}}}{\sqrt{\mathfrak{f}_{1,1} \mathfrak{f}_{2,2}}}\left(\left[\mathfrak{G}_{1,2}-\mathfrak{f}_{1,2}-\boldsymbol{B}_{1,2}\right]-\frac{1}{2} \frac{\mathfrak{f}_{1,2}}{\mathfrak{f}_{1,1}}\left[\mathfrak{G}_{1,1}-\mathfrak{f}_{1,1}-\boldsymbol{B}_{1,1}\right]-\frac{1}{2} \frac{\mathfrak{f}_{1,2}}{\mathfrak{f}_{2,2}}\left[\mathfrak{G}_{2,2}-\mathfrak{f}_{2,2}-\boldsymbol{B}_{2,2}\right]\right)
$$

are asymptotically equivalent in the sense that if one of the two converges weakly in $\ell_{\mathbb{C}^{d \times d}}^{\infty}\left([0,1]^{2}\right)$, then so does the other. The assertion then follows by Theorem 3.3. Slutzky's lemma and the Continuous Mapping Theorem.

\subsection{Auxiliary Lemmas}

In this section we state multivariate versions of the auxiliary lemmas from Section 7.4 in Kley et al. (2015). Note that Lemma 7.4 is unaltered and therefore stated without proof. The remaining lemmas are adapted to the multivariate quantities and proofs or directions on how to adapt the proofs in Kley et al. (2015) are collected in the end of this section.

For the statement of Lemma 7.4, we define the Orlicz norm [see e.g. van der Vaart and Wellner (1996), Chapter 2.2] of a real-valued random variable $Z$ as

$$
\|Z\|_{\Psi}=\inf \{C>0: \mathbb{E} \Psi(|Z| / C) \leq 1\}
$$

where $\Psi: \mathbb{R}^{+} \rightarrow \mathbb{R}^{+}$may be any non-decreasing, convex function with $\Psi(0)=0$.

For the statement of Lemmas 7.5, 7.7, and 7.10 we define, for any Borel set $A$,

$$
d_{n}^{j}(\omega ; A):=\sum_{t=0}^{n-1} I\left\{X_{t, j} \in A\right\} \mathrm{e}^{-\mathrm{i} t \omega}
$$

Lemma 7.4. Let $\left\{\mathbb{G}_{t}: t \in T\right\}$ be a separable stochastic process with $\left\|\mathbb{G}_{s}-\mathbb{G}_{t}\right\|_{\Psi} \leq$ $C d(s, t)$ for all $s, t$ with $d(s, t) \geq \bar{\eta} / 2 \geq 0$. Denote by $D(\epsilon, d)$ the packing number of the metric space $(T, d)$. Then, for any $\delta>0, \eta \geq \bar{\eta}$, there exists a random variable $S_{1}$ and a constant $K<\infty$ such that

$$
\begin{aligned}
\sup _{d(s, t) \leq \delta}\left|\mathbb{G}_{s}-\mathbb{G}_{t}\right| & \leq S_{1}+2 \sup _{d(s, t) \leq \bar{\eta}, t \in \tilde{T}}\left|\mathbb{G}_{s}-\mathbb{G}_{t}\right| \quad \text { and } \\
\left\|S_{1}\right\|_{\Psi} & \leq K\left[\int_{\bar{\eta} / 2}^{\eta} \Psi^{-1}(D(\epsilon, d)) d \epsilon+(\delta+2 \bar{\eta}) \Psi^{-1}\left(D^{2}(\eta, d)\right)\right],
\end{aligned}
$$

where the set $\tilde{T}$ contains at most $D(\bar{\eta}, d)$ points. In particular, by Markov's inequality [cf. van der Vaart and Wellner (1996), p. 96],

$$
\mathbb{P}\left(\left|S_{1}\right|>x\right) \leq\left(\Psi\left(x\left[8 K\left(\int_{\bar{\eta} / 2}^{\eta} \Psi^{-1}(D(\epsilon, d)) d \epsilon+(\delta+2 \bar{\eta}) \Psi^{-1}\left(D^{2}(\eta, d)\right)\right)\right]^{-1}\right)\right)^{-1} .
$$

for any $x>0$. 
Lemma 7.5. Let $\boldsymbol{X}_{0}, \ldots, \boldsymbol{X}_{n-1}$, where $\boldsymbol{X}_{t}=\left(X_{t, 1}, \ldots, X_{t, d}\right)$, be the finite realization of a strictly stationary process with $X_{0, j} \sim U[0,1], j=1, \ldots, d$. Let (W) hold. For $x=\left(x_{1}, x_{2}\right)$ let $\hat{H}_{n}^{j_{1}, j_{2}}(x ; \omega):=\sqrt{n b_{n}}\left(\hat{G}_{n}^{j_{1}, j_{2}}\left(x_{1}, x_{2} ; \omega\right)-\mathbb{E}\left[\hat{G}_{n}^{j_{1}, j_{2}}\left(x_{1}, x_{2} ; \omega\right)\right]\right)$. Let $d_{n}^{j}(\omega ; A)$ be defined as in (40). Assume that, for $p=1, \ldots, P$, there exist a constant $C$ and a function $g: \mathbb{R}^{+} \rightarrow \mathbb{R}^{+}$, both independent of $\omega_{1}, \ldots, \omega_{p} \in \mathbb{R}, n$ and $A_{1}, \ldots, A_{p}$, such that

$$
\left|\operatorname{cum}\left(d_{n}^{j_{1}}\left(\omega_{1} ; A_{1}\right), \ldots, d_{n}^{j_{p}}\left(\omega_{p} ; A_{p}\right)\right)\right| \leq C\left(\left|\Delta_{n}\left(\sum_{i=1}^{p} \omega_{i}\right)\right|+1\right) g(\varepsilon)
$$

for any indices $j_{1}, \ldots, j_{p} \in\{1, \ldots, d\}$ and intervals $A_{1}, \ldots, A_{p}$ with $\min _{k} \mathbb{P}\left(X_{0, j_{k}} \in A_{k}\right) \leq$ $\varepsilon$. Then, there exists a constant $K$ (depending on $C, L, g$ only) such that

$$
\sup _{\omega \in \mathbb{R}} \sup _{\|a-b\|_{1} \leq \varepsilon} \mathbb{E}\left|\hat{H}_{n}^{j_{1}, j_{2}}(a ; \omega)-\hat{H}_{n}^{j_{1}, j_{2}}(b ; \omega)\right|^{2 L} \leq K \sum_{\ell=0}^{L-1} \frac{g^{L-\ell}(\varepsilon)}{\left(n b_{n}\right)^{\ell}}
$$

for all $\varepsilon$ with $g(\varepsilon)<1$ and all $L=1, \ldots, P$.

Lemma 7.6. Under the assumptions of Theorem 3.3, the derivative

$$
\left(\tau_{1}, \tau_{2}\right) \mapsto \frac{\mathrm{d}^{k}}{\mathrm{~d} \omega^{k}} \mathrm{f}^{j_{1}, j_{2}}\left(\omega ; \tau_{1}, \tau_{2}\right)
$$

exists and satisfies, for any $k \in \mathbb{N}_{0}$ and some constants $C, d$ that are independent of $a=\left(a_{1}, a_{2}\right), b=\left(b_{1}, b_{2}\right)$, but may depend on $k$,

$$
\sup _{\omega \in \mathbb{R}}\left|\frac{\mathrm{d}^{k}}{\mathrm{~d} \omega^{k}} \mathfrak{f}^{j_{1}, j_{2}}\left(\omega ; a_{1}, a_{2}\right)-\frac{\mathrm{d}^{k}}{\mathrm{~d} \omega^{k}} \mathfrak{f}^{j_{1}, j_{2}}\left(\omega ; b_{1}, b_{2}\right)\right| \leq C\|a-b\|_{1}\left(1+\left|\log \|a-b\|_{1}\right|\right)^{D} .
$$

Lemma 7.7. Let the strictly stationary process $\left(\boldsymbol{X}_{t}\right)_{t \in \mathbb{Z}}$ satisfy Assumption $(C)$. Let $d_{n}^{j}(\omega ; A)$ be defined as in $(40)$. Let $A_{1}, \ldots, A_{p} \subset[0,1]$ be intervals, and let

$$
\varepsilon:=\min _{k=1, \ldots, p} \mathbb{P}\left(X_{0, j_{k}} \in A_{k}\right) .
$$

Then, for any $p$-tuple $\omega_{1}, \ldots, \omega_{p} \in \mathbb{R}$ and $j_{1}, \ldots, j_{p} \in\{1, \ldots, d\}$,

$$
\left|\operatorname{cum}\left(d_{n}^{j_{1}}\left(\omega_{1} ; A_{1}\right), \ldots, d_{n}^{j_{p}}\left(\omega_{p} ; A_{p}\right)\right)\right| \leq C\left(\left|\Delta_{n}\left(\sum_{i=1}^{p} \omega_{i}\right)\right|+1\right) \varepsilon(|\log \varepsilon|+1)^{D},
$$

where $\Delta_{n}(\lambda):=\sum_{t=0}^{n-1} e^{\mathrm{it \lambda}}$ and the constants $C, D$ depend only on $K, p$, and $\rho$ [with $\rho$ from condition $(C)]$.

Lemma 7.8. Assume that $\left(\boldsymbol{X}_{t}\right)_{t \in \mathbb{Z}}$ is a strictly stationary process satisfying Assumption $(C)$ and $X_{0, j} \sim U[0,1]$. Denote by $\hat{F}_{n, j}$ the empirical distribution function of $X_{0, j}, \ldots, X_{n-1, j}$. Then, for any $k \in \mathbb{N}$, there exists a constant $d_{k}$ depending only on $k$, such that

$$
\begin{aligned}
\sup _{x, y \in[0,1],|x-y| \leq \delta_{n}} \sqrt{n} \mid \hat{F}_{n, j}(x)-\hat{F}_{n, j}(y)- & (x-y) \mid \\
& =O_{P}\left(\left(n^{2} \delta_{n}+n\right)^{1 / 2 k}\left(\delta_{n}\left|\log \delta_{n}\right|^{d_{k}}+n^{-1}\right)^{1 / 2}\right),
\end{aligned}
$$

as $\delta_{n} \rightarrow 0$. 
Lemma 7.9. Let $\boldsymbol{X}_{0}, \ldots, \boldsymbol{X}_{n-1}$, where $\boldsymbol{X}_{t}=\left(X_{t, 1}, \ldots, X_{t, d}\right)$, be the finite realization of a strictly stationary process satisfying $(C)$ and $X_{0, j} \sim U[0,1], j=1, \ldots, d$. Then,

$$
\sup _{j=1, \ldots, d} \sup _{\tau \in[0,1]}\left|\hat{F}_{n, j}^{-1}(\tau)-\tau\right|=O_{P}\left(n^{-1 / 2}\right) .
$$

Lemma 7.10. Let the strictly stationary process $\left(\boldsymbol{X}_{t}\right)_{t \in \mathbb{Z}}$ satisfy $(C)$ and $X_{0, j} \sim U[0,1]$. Let $d_{n}^{j}(\omega ; A)$ be defined as in (40). Then, for any $k \in \mathbb{N}$,

$$
\sup _{j=1, \ldots, d} \sup _{\omega \in \mathcal{F}_{n}} \sup _{y \in[0,1]}\left|d_{n}^{j}(\omega ;[0, y])\right|=O_{P}\left(n^{1 / 2+1 / k}\right) .
$$

Lemma 7.11. Under the assumptions of Theorem 7.1. let $\delta_{n}$ be a sequence of nonnegative real numbers. Assume that there exists $\gamma \in(0,1)$, such that $\delta_{n}=O\left(\left(n b_{n}\right)^{-1 / \gamma}\right)$. Then,

$$
\sup _{j_{1}, j_{2}, \in\{1, \ldots, d\}} \sup _{\omega \in \mathbb{R}} \sup _{\substack{u, v \in[0,1]^{2} \\\|u-v\|_{1} \leq \delta_{n}}}\left|\hat{H}_{n}^{j_{1}, j_{2}}(u ; \omega)-\hat{H}_{n}^{j_{1}, j_{2}}(v ; \omega)\right|=o_{P}(1) .
$$

Proof of Lemma 7.4. The lemma is stated unaltered as in Kley et al. (2015). The proof can be found in Section 8.3.1 of the Online Appendix of Kley et al. (2015).

Proof of Lemma 7.5. Along the same lines of the proof of the univariate version (Section 8.3.2 in Kley et al. (2015)) we can proof

$$
\mathbb{E}\left|\hat{H}_{n}^{j_{1}, j_{2}}(a ; \omega)-\hat{H}_{n}^{j_{1}, j_{2}}(b ; \omega)\right|^{2 L}=\sum_{\substack{\left\{\nu_{1}, \ldots, \nu_{R}\right\} \\\left|\nu_{j}\right| \geq 2, j=1, \ldots, R}} \prod_{r=1}^{R} \mathcal{D}_{a, b}\left(\nu_{r}\right)
$$

with the summation running over all partitions $\left\{\nu_{1}, \ldots, \nu_{R}\right\}$ of $\{1, \ldots, 2 L\}$ such that each set $\nu_{j}$ contains at least two elements, and

$$
\begin{aligned}
\mathcal{D}_{a, b}(\xi):= & \sum_{\ell_{\xi_{1}}, \ldots, \ell_{\xi_{q}} \in\{1,2\}} n^{-3 q / 2} b_{n}^{q / 2}\left(\prod_{m \in \xi} \sigma_{\ell_{m}}\right) \\
& \times \sum_{s_{\xi_{1}}, \ldots, s_{\xi_{q}}=1}^{n-1}\left(\prod_{m \in \xi} W_{n}\left(\omega-2 \pi s_{m} / n\right)\right) \operatorname{cum}\left(D_{\ell_{m},(-1)^{m-1} s_{m}}: m \in \xi\right),
\end{aligned}
$$

for any set $\xi:=\left\{\xi_{1}, \ldots, \xi_{q}\right\} \subset\{1, \ldots, 2 L\}, q:=|\xi|$, and

$$
D_{\ell, s}:=d_{n}^{j_{1}}\left(2 \pi s / n ; M_{1}(\ell)\right) d_{n}^{j_{2}}\left(-2 \pi s / n ; M_{2}(\ell)\right), \quad \ell=1,2, \quad s=1, \ldots, n-1,
$$

with the sets $M_{1}(1), M_{2}(2), M_{2}(1), M_{1}(2)$ and the signs $\sigma_{\ell} \in\{-1,1\}$ defined as

$$
\begin{aligned}
\sigma_{1} & :=2 I\left\{a_{1}>b_{1}\right\}-1, & \sigma_{2} & :=2 I\left\{a_{2}>b_{2}\right\}-1, \\
M_{1}(1) & :=\left(a_{1} \wedge b_{1}, a_{1} \vee b_{1}\right], & M_{2}(2) & :=\left(a_{2} \wedge b_{2}, a_{2} \vee b_{2}\right], \\
M_{2}(1) & := \begin{cases}{\left[0, a_{2}\right]} & b_{2} \geq a_{2} \\
{\left[0, b_{2}\right]} & a_{2}>b_{2},\end{cases} & M_{1}(2) & := \begin{cases}{\left[0, b_{1}\right]} & b_{2} \geq a_{2} \\
{\left[0, a_{1}\right]} & a_{2}>b_{2} .\end{cases}
\end{aligned}
$$


Employing assumption (41), we can further prove, by following the arguments of the univariate version, that

$$
\sup _{\substack{\xi \subset\{1, \ldots, 2 L\} \\|\xi|=q}} \sup _{\|a-b\|_{1} \leq \varepsilon}\left|\mathcal{D}_{a, b}(\xi)\right| \leq C\left(n b_{n}\right)^{1-q / 2} g(\varepsilon), \quad 2 \leq q \leq 2 L .
$$

The lemma then follows, by observing that

$$
\left|\prod_{r=1}^{R} \mathcal{D}_{a, b}\left(\nu_{r}\right)\right| \leq C g^{R}(\varepsilon)\left(n b_{n}\right)^{R-L}
$$

for any partition in 42 [note that $\sum_{r=1}^{R}\left|\nu_{r}\right|=2 L$ ].

Proof of Lemma 7.6. Note that

$$
\begin{aligned}
& \operatorname{cum}\left(I\left\{X_{0, j_{1}} \leq q_{j_{1}}\left(a_{1}\right)\right\}, I\left\{X_{k, j_{2}} \leq q_{j_{2}}\left(a_{2}\right)\right\}\right)-\operatorname{cum}\left(I\left\{X_{0, j_{1}} \leq q_{j_{1}}\left(b_{1}\right)\right\}, I\left\{X_{k, j_{2}} \leq q_{j_{2}}\left(b_{2}\right)\right\}\right) \\
& =\sigma_{1} \operatorname{cum}\left(I\left\{F_{j_{1}}\left(X_{0, j_{1}}\right) \in M_{1}(1)\right\}, I\left\{F_{j_{2}}\left(X_{k, j_{2}}\right) \in M_{2}(1)\right\}\right) \\
& \quad+\sigma_{2} \operatorname{cum}\left(I\left\{F_{j_{1}}\left(X_{0, j_{1}}\right) \in M_{1}(2)\right\}, I\left\{F_{j_{2}}\left(X_{k, j_{2}}\right) \in M_{2}(2)\right\}\right)
\end{aligned}
$$

with the sets $M_{1}(1), M_{2}(2), M_{2}(1), M_{1}(2)$ and the signs $\sigma_{\ell} \in\{-1,1\}$ defined in (43).

From the fact that $\lambda\left(M_{j}(j)\right) \leq\|a-b\|_{1}$ for $j=1,2$, we conclude that

$$
\begin{aligned}
& \left|\frac{\mathrm{d}^{\ell}}{\mathrm{d} \omega^{\ell}} \mathfrak{f}^{j_{1}, j_{2}}\left(\omega ; a_{1}, a_{2}\right)-\frac{\mathrm{d}^{\ell}}{\mathrm{d} \omega^{\ell}} \mathfrak{f}^{j_{1}, j_{2}}\left(\omega ; b_{1}, b_{2}\right)\right| \\
& \leq \sum_{k \in \mathbb{Z}}|k|^{\ell}\left|\operatorname{cum}\left(I\left\{F_{j_{1}}\left(X_{0, j_{1}}\right) \in M_{1}(1)\right\}, I\left\{F_{j_{2}}\left(X_{k, j_{2}}\right) \in M_{2}(1)\right\}\right)\right| \\
& \quad+\sum_{k \in \mathbb{Z}}|k|^{\ell}\left|\operatorname{cum}\left(I\left\{F_{j_{1}}\left(X_{0, j_{1}}\right) \in M_{1}(2)\right\}, I\left\{F_{j_{2}}\left(X_{k, j_{2}}\right) \in M_{2}(2)\right\}\right)\right| \\
& \leq 4 \sum_{k=0}^{\infty} k^{\ell}\left(\left(K \rho^{\ell}\right) \wedge\|a-b\|_{1}\right) .
\end{aligned}
$$

The assertion then follows by after some algebraic manipulations.

Proof of Lemma 7.7. Similar to (8.27) in Kley et al. (2015) we have, by the definition of cumulants and strict stationarity,

$$
\begin{aligned}
& \operatorname{cum}\left(d_{n}^{j_{1}}\left(\omega_{1} ; A_{1}\right), \ldots, d_{n}^{j_{p}}\left(\omega_{p} ; A_{p}\right)\right) \\
&=\sum_{u_{2}, \ldots, u_{p}=-n}^{n} \operatorname{cum}\left(I\left\{X_{0, j_{1}} \in A_{1}\right\}, I\left\{X_{u_{2}, j_{2}} \in A_{2}\right\} \ldots, I\left\{X_{u_{p}, j_{p}} \in A_{p}\right\}\right) \exp \left(-\mathrm{i} \sum_{j=2}^{p} \omega_{j} u_{j}\right) \\
& \quad \times \sum_{t_{1}=0}^{n-1} \exp \left(-\mathrm{i} t_{1} \sum_{j=1}^{p} \omega_{j}\right) I_{\left\{0 \leq t_{1}+u_{2}<n\right\}} \cdots I_{\left\{0 \leq t_{1}+u_{p}<n\right\}} \cdot \quad(44)
\end{aligned}
$$

By Lemma 8.1 in Kley et al. (2015),

$$
\left|\Delta_{n}\left(\sum_{j=1}^{p} \omega_{j}\right)-\sum_{t_{1}=0}^{n-1} \exp \left(-\mathrm{i} t_{1} \sum_{j=1}^{p} \omega_{j}\right) I\left\{0 \leq t_{1}+u_{2}<n\right\} \cdots I\left\{0 \leq t_{1}+u_{p}<n\right\}\right| \leq 2 \sum_{j=2}^{p}\left|u_{j}\right| .
$$


Following the arguments for the proof of (8.29) in Kley et al. (2015), we further have, for any $p+1$ intervals $A_{0}, \ldots, A_{p} \subset \mathbb{R}$, any indices $j_{0}, \ldots, j_{p} \in\{1, \ldots, d\}$, and any $p$-tuple $\kappa:=\left(\kappa_{1}, \ldots, \kappa_{p}\right) \in \mathbb{R}_{+}^{p}, p \geq 2$, that

$$
\begin{aligned}
\sum_{k_{1}, \ldots, k_{p}=-\infty}^{\infty}\left(1+\sum_{\ell=1}^{p}\left|k_{\ell}\right|^{\kappa_{\ell}}\right) \mid \operatorname{cum}\left(I\left\{X_{k_{1}, j_{1}} \in A_{1}\right\}, \ldots, I\left\{X_{k_{p}, j_{p}}\right.\right. & \left.\left.\in A_{p}\right\}, I\left\{X_{0, j_{0}} \in A_{0}\right\}\right) \mid \\
& \leq C \varepsilon(|\log \varepsilon|+1)^{d}
\end{aligned}
$$

To this end, define $k_{0}=0$, consider the set

$$
T_{m}:=\left\{\left(k_{1}, \ldots, k_{p}\right) \in \mathbb{Z}^{p}\left|\max _{i, j=0, \ldots, p}\right| k_{i}-k_{j} \mid=m\right\},
$$

and note that $\left|T_{m}\right| \leq c_{p} m^{p-1}$ for some constant $c_{p}$. From the definition of cumulants and some simple algebra we get the bound

$$
\left|\operatorname{cum}\left(I\left\{X_{t_{1}, j_{1}} \in A_{1}\right\}, \ldots, I\left\{X_{t_{p}, j_{p}} \in A_{p}\right\}\right)\right| \leq C \min _{i=1, \ldots, p} P\left(X_{0, j_{i}} \in A_{i}\right) .
$$

With this bound and condition (C) we obtain, employing the above notation, that

$$
\begin{aligned}
& \sum_{k_{1}, \ldots, k_{p}=-\infty}^{\infty}\left(1+\sum_{j=1}^{p}\left|k_{\ell}\right|^{\kappa_{\ell}}\right)\left|\operatorname{cum}\left(I\left\{X_{k_{1}, j_{1}} \in A_{1}\right\}, \ldots, I\left\{X_{k_{p}, j_{p}} \in A_{p}\right\}, I_{\left\{X_{0, j_{0}} \in A_{0}\right\}}\right)\right| \\
= & \sum_{m=0}^{\infty} \sum_{\left(k_{1}, \ldots, k_{p}\right) \in T_{m}}\left(1+\sum_{\ell=1}^{p}\left|k_{\ell}\right|^{\kappa_{\ell}}\right)\left|\operatorname{cum}\left(I\left\{X_{k_{1}, j_{1}} \in A_{1}\right\}, \ldots, I\left\{X_{k_{p}, j_{p}} \in A_{p}\right\}, I\left\{X_{0, j_{0}} \in A_{0}\right\}\right)\right| \\
\leq & \sum_{m=0}^{\infty} \sum_{\left(k_{1}, \ldots, k_{p}\right) \in T_{m}}\left(1+p m^{\max _{j} \kappa_{j}}\right)\left(\rho^{m} \wedge \varepsilon\right) K_{p} \leq C_{p} \sum_{m=0}^{\infty}\left(\rho^{m} \wedge \varepsilon\right)\left|T_{m}\right| m^{\max _{j} \kappa_{j}} .
\end{aligned}
$$

For $\varepsilon \geq \rho$, (46) then follows trivially. For $\varepsilon<\rho$, set $m_{\varepsilon}:=\log \varepsilon / \log \rho$ and note that $\rho^{m} \leq \varepsilon$ if and only if $m \geq m_{\varepsilon}$. Thus,

$$
\sum_{m=0}^{\infty}\left(\rho^{m} \wedge \varepsilon\right) m^{u} \leq \sum_{m \leq m_{\varepsilon}} m^{u} \varepsilon+\sum_{m>m_{\varepsilon}} m^{u} \rho^{m} \leq C\left(\varepsilon m_{\varepsilon}^{u+1}+\rho^{m_{\varepsilon}} \sum_{m=0}^{\infty}\left(m+m_{\varepsilon}\right)^{u} \rho^{m}\right) .
$$

The fact that $\rho^{m_{\varepsilon}}=\varepsilon$ completes the proof of the desired inequality (46). The assertion follows from (44), 45), (46) and the triangle inequality.

Proofs of Lemmas 7.8, 7.9 and 7.10. Note that the component processes $\left(X_{t, j}\right)$ are stationary and fulfill assumption (C) in Kley et al. (2015), for every $j=1, \ldots, d$. The assertion then follow from the univariate versions (i. e., Lemma 8.6, 7.5 and 7.6 in Kley et al. (2015), respectively), as the dimension $d$ does not depend on $n$.

Proof of Lemma 7.11. Assume, without loss of generality, that $n^{-1}=o\left(\delta_{n}\right)$ [otherwise, enlarge the supremum by considering $\left.\tilde{\delta}_{n}:=\max \left(n^{-1}, \delta_{n}\right)\right]$. With the notation $a=\left(a_{1}, a_{2}\right)$ and $b=\left(b_{1}, b_{2}\right)$, we have

$$
\hat{H}_{n}^{j_{1}, j_{2}}(a ; \omega)-\hat{H}_{n}^{j_{1}, j_{2}}(b ; \omega)=b_{n}^{1 / 2} n^{-1 / 2} \sum_{s=1}^{n-1} W_{n}(\omega-2 \pi s / n)\left(K_{s, n}(u, v)-\mathbb{E} K_{s, n}(u, v)\right)
$$


where, with $d_{n, U}^{j}$ defined in 33 ,

$$
\begin{aligned}
K_{s, n}(a, b):= & n^{-1}\left(d_{n, U}^{j_{1}}\left(2 \pi s / n ; u_{1}\right) d_{n, U}^{j_{2}}\left(-2 \pi s / n ; u_{2}\right)-d_{n, U}^{j_{1}}\left(2 \pi s / n ; v_{1}\right) d_{n, U}^{j_{2}}\left(-2 \pi s / n ; v_{2}\right)\right) \\
= & d_{n, U}^{j_{1}}\left(2 \pi s / n ; u_{1}\right) n^{-1}\left[d_{n, U}^{j_{2}}\left(-2 \pi s / n ; u_{2}\right)-d_{n, U}^{j_{2}}\left(-2 \pi s / n ; v_{2}\right)\right] \\
& +d_{n, U}^{j_{2}}\left(-2 \pi s / n ; v_{2}\right) n^{-1}\left[d_{n, U}^{j_{1}}\left(2 \pi s / n ; u_{1}\right)-d_{n, U}^{j_{1}}\left(2 \pi s / n ; v_{1}\right)\right] .
\end{aligned}
$$

By Lemma 7.10, we have, for any $k \in \mathbb{N}$,

$$
\sup _{y \in[0,1]} \sup _{\omega \in \mathcal{F}_{n}}\left|d_{n, U}^{j}(\omega ; y)\right|=O_{P}\left(n^{1 / 2+1 / k}\right) .
$$

Employing Lemma 7.8, we have, for any $\ell \in \mathbb{N}$ and $j=1, \ldots, d$,

$$
\begin{aligned}
& \sup _{\omega \in \mathbb{R}} \sup _{y \in[0,1]} \sup _{x:|x-y| \leq \delta_{n}} n^{-1}\left|d_{n, U}^{j}(\omega ; x)-d_{n, U}^{j}(\omega ; y)\right| \\
& \leq \sup _{y \in[0,1]} \sup _{x:|x-y| \leq \delta_{n}} n^{-1} \sum_{t=0}^{n-1}\left|I\left\{F_{j}\left(X_{t, j}\right) \leq x\right\}-I\left\{F_{j}\left(X_{t, j}\right) \leq y\right\}\right| \\
& \leq \sup _{y \in[0,1]} \sup _{x:|x-y| \leq \delta_{n}}\left|\hat{F}_{n, j}(x \vee y)-\hat{F}_{n, j}(x \wedge y)-x \vee y+x \wedge y\right|+C \delta_{n} \\
& =O_{P}\left(\rho_{n}\left(\delta_{n}, \ell\right)+\delta_{n}\right)
\end{aligned}
$$

with $\rho_{n}\left(\delta_{n}, \ell\right):=n^{-1 / 2}\left(n^{2} \delta_{n}+n\right)^{1 / 2 \ell}\left(\delta_{n}\left|\log \delta_{n}\right|^{D_{\ell}}+n^{-1}\right)^{1 / 2}, \hat{F}_{n, j}$ denoting the empirical distribution function of $F_{j}\left(X_{0, j}\right), \ldots, F_{j}\left(X_{n-1, j}\right)$, and $d_{\ell}$ being a constant depending only on $\ell$. Combining these arguments and observing that

$$
\sup _{\omega \in \mathbb{R}} \sum_{s=1}^{n-1}\left|W_{n}(\omega-2 \pi s / n)\right|=O(n)
$$

yields

$$
\sup _{\substack{\omega \in \mathbb{R} \\\left\|u, v \in[0,1]^{2}\\\right\| u-v \|_{1} \leq \delta_{n}}}\left|\sum_{s=1}^{n-1} W_{n}(\omega-2 \pi s / n) K_{s, n}(u, v)\right|=O_{P}\left(n^{3 / 2+1 / k}\left(\rho\left(\delta_{n}, \ell\right)+\delta_{n}\right)\right) .
$$

With $M_{i}(j), i, j=1,2$, as defined in $(43)$, we have

$$
\begin{aligned}
& \sup _{\|a-b\|_{1} \leq \delta_{n}} \sup _{s=1, \ldots, n-1}\left|\mathbb{E} K_{s, n}(a, b)\right| \\
& \quad \leq n^{-1} \sup _{\|a-b\|_{1} \leq \delta_{n}} \sup _{s=1, \ldots, n-1}\left|\operatorname{cum}\left(d_{n, U}^{j_{1}}\left(2 \pi s / n ; M_{1}(1)\right), d_{n, U}^{j_{2}}\left(-2 \pi s / n ; M_{2}(1)\right)\right)\right| \\
& \quad+n^{-1} \sup _{\|a-b\|_{1} \leq \delta_{n}} \sup _{s=1, \ldots, n-1}\left|\operatorname{cum}\left(d_{n, U}^{j_{1}}\left(2 \pi s / n ; M_{1}(2)\right), d_{n, U}^{j_{2}}\left(-2 \pi s / n ; M_{2}(2)\right)\right)\right|
\end{aligned}
$$

where we have used $\mathbb{E} d_{n, U}^{j}(2 \pi s / n ; M)=0$. Lemma 7.7 and $\lambda\left(M_{j}(j)\right) \leq \delta_{n}$, for $j=1,2$ (with $\lambda$ denoting the Lebesgue measure over $\mathbb{R}$ ) yield

$\sup _{\|a-b\|_{1} \leq \delta_{n}} \sup _{s=1, \ldots, n-1}\left|\operatorname{cum}\left(d_{n}^{j_{1}}\left(2 \pi s / n ; M_{1}(j)\right), d_{n}^{j_{2}}\left(-2 \pi s / n ; M_{2}(j)\right)\right)\right| \leq C(n+1) \delta_{n}\left(1+\left|\log \delta_{n}\right|\right)^{D}$, 
It follows that the right-hand side in $(50)$ is $O\left(\delta_{n}\left|\log \delta_{n}\right|^{D}\right)$. Therefore, by (48), we obtain

$$
\sup _{\omega \in \mathbb{R}} \sup _{\|a-b\|_{1} \leq \delta_{n}}\left|b_{n}^{1 / 2} n^{-1 / 2} \sum_{s=1}^{n-1} W_{n}(\omega-2 \pi s / n) \mathbb{E} K_{s, n}(a, b)\right|=O\left(\left(n b_{n}\right)^{1 / 2} \delta_{n}|\log n|^{D}\right) .
$$

In view of the assumption that $n^{-1}=o\left(\delta_{n}\right)$, we have $\delta_{n}=O\left(n^{1 / 2} \rho_{n}\left(\delta_{n}, \ell\right)\right)$, which, in combination with 49 , yields

$$
\begin{aligned}
& \sup _{\omega \in \mathbb{R}} \sup _{\|a-b\|_{1} \leq \delta_{n}}\left|\hat{H}_{n}^{j_{1}, j_{2}}(a ; \omega)-\hat{H}_{n}^{j_{1}, j_{2}}(b ; \omega)\right| \\
& =O_{P}\left(\left(n b_{n}\right)^{1 / 2}\left[n^{1 / 2+1 / k}\left(\rho_{n}\left(\delta_{n}, \ell\right)+\delta_{n}\right)+\delta_{n}\left|\log \delta_{n}\right|^{D}\right]\right) \\
& =O_{P}\left(\left(n b_{n}\right)^{1 / 2} n^{1 / 2+1 / k} \rho_{n}\left(\delta_{n}, \ell\right)\right) \\
& =O_{P}\left(\left(n b_{n}\right)^{1 / 2} n^{1 / k+1 / \ell}\left(n^{-1} \vee \delta_{n}(\log n)^{D_{\ell}}\right)^{1 / 2}\right)=o_{P}(1) .
\end{aligned}
$$

The $o_{P}(1)$ holds, as we have, for arbitrary $k$ and $\ell$,

$$
O\left(\left(n b_{n}\right)^{1 / 2} n^{1 / k+1 / \ell} \delta_{n}^{1 / 2}(\log n)^{D_{\ell} / 2}\right)=O\left(\left(n b_{n}\right)^{1 / 2-1 / 2 \gamma} n^{1 / k+1 / \ell}(\log n)^{D_{\ell} / 2}\right) .
$$

The assumptions on $b_{n}$ imply $\left(n b_{n}\right)^{1 / 2-1 / 2 \gamma}=o\left(n^{-\kappa}\right)$ for some $\kappa>0$, such that this latter quantity is $o(1)$ for $k, \ell$ sufficiently large. The term $\left(n b_{n}\right)^{1 / 2} n^{1 / k+1 / \ell} n^{-1 / 2}$ is handled in a similar fashion. This concludes the proof. 


